\title{
An Integral PBW Basis of the Quantum Affine Algebra of Type $A_{2}^{(2)}$
}

By

Tatsuya AKASAKA*

\begin{abstract}
We construct an integral PBW basis and an integral crystal basis of the quantum affine algebra of type $A_{2}^{(2)}$.
\end{abstract}

\section{$\S 1 . \quad$ Introduction}

For the study of the precise structure of a quantum affine algebra $\mathbf{U}$, the construction of a good basis is an important problem.

Damiani constructed a PBW basis in the $A_{1}^{(1)}$ case [Da1]. Generalizing it, Beck constructed one in the untwisted case [B2]. Their bases are considered over $\mathbf{Q}(q)$.

However, to study the representations of $\mathbf{U}$ when $q$ is specialized at the roots of unity, one needs a $\mathbf{Z}\left[q, q^{-1}\right]$-basis of a certain $\mathbf{Z}\left[q, q^{-1}\right]$-subalgebra $\mathbf{U}_{\mathbf{Z}}$ of $\mathbf{U}$ (see [L, Part 5]). We call such a basis an integral basis of $\mathbf{U}$. Also, to deal with the structure of the Grothendieck group of level 0 representations of $\mathbf{U}$, an integral basis is needed (see [K2]).

Around 1990, Kashiwara and Lusztig introduced the notion of a global crystal basis (associated with a crystal basis) and a canonical basis respectively and proved the existence and uniqueness [K1], [L2]. It turned out that these two notions are equivalent [GL].

Let $\mathbf{U}^{+}$be the positive part of $\mathbf{U}$ and let $\mathbf{U}_{\mathbf{Z}}^{+}=\mathbf{U}_{\mathbf{Z}} \cap \mathbf{U}^{+}$. Let $\mathbf{B}$ be the global crystal basis of $\mathbf{U}^{+}$and let $L$ be the $\mathbf{Z}\left[q^{-1}\right]$-lattice generated by $\mathbf{B}$.

Communicated by M. Kashiwara. Received April 27, 2001.

2000 Mathematics Subject Classification(s): 17B37.

*International Institute for Advanced Studies, Kyoto 619-0225, Japan.

e-mail: akasaka@iias.or.jp 
Then, $\mathbf{B}$ is a $\mathbf{Q}(q)$-basis of $\mathbf{U}^{+}$, a $\mathbf{Z}\left[q, q^{-1}\right]$-basis of $\mathbf{U}_{\mathbf{Z}}^{+}$, a $\mathbf{Z}\left[q^{-1}\right]$-basis of $L$, a $\mathbf{Z}[q]$-basis of $\bar{L}$, and a $\mathbf{Z}$-basis of $L \cap \bar{L}$. Here, - is the Q-linear involution of $\mathbf{U}^{+}$given by $\bar{q}=q^{-1}, \overline{e_{i}}=e_{i}$.

An integral crystal basis $B$ of $\mathbf{U}^{+}$is, by definition, a $\mathbf{Z}\left[q^{-1}\right]$-basis of $L$ that coincides with $\mathbf{B}$ modulo $q^{-1} L$. Let $T$ be the transformation matrix with coefficients in $\mathbf{Z}\left[q, q^{-1}\right]$ between $B$ and $\bar{B}$, i.e. $B=T \bar{B}$ in the matrix form. There is a unique invertible matrix $A$ with coefficients in $\mathbf{Z}\left[q^{-1}\right]$ such that $T=A^{-1} \bar{A}$. Then we have $\mathbf{B}=A B$. Thus, we can recover $\mathbf{B}$ from $B$.

Using the results in $[\mathrm{CP}]$, Beck, Chari, and Pressley constructed an integral PBW basis and an integral crystal basis in the simply-laced case [BCP].

The purpose of this paper is to construct an integral PBW basis and an integral crystal basis in the $A_{2}^{(2)}$ case.

Let us take a closer look at the results of this paper. Let $\alpha_{0}$ be the long simple root and $\alpha_{1}$ the short simple root of type $A_{2}^{(2)}$. Let $\delta=\alpha_{0}+2 \alpha_{1}$, the smallest positive imaginary root. Let $\mathbf{U}$ be the quantum affine algebra of type $A_{2}^{(2)}$ and let $\mathbf{U}_{\mathbf{Z}}$ be its integral form (see Definition 2.12). Here, $\mathbf{U}$ is an algebra over $\mathbf{Q}\left(q_{1}\right)$ and $\mathbf{U}_{\mathbf{Z}}$ is an algebra over $\mathbf{Z}\left[q_{1}, q_{1}^{-1}\right]$ (see Section 2 for $q_{1}=q^{1 / 2}$ ). Let $\mathbf{U}^{+}$be the positive part of $\mathbf{U}$ and let $\mathbf{U}_{\mathbf{Z}}^{+}=\mathbf{U}_{\mathbf{Z}} \cap \mathbf{U}^{+}$.

We divide the set of positive real roots into two parts:

$$
R_{r e}^{+}(>)=\left\{n \delta+\alpha_{1},(2 n+2) \delta-\alpha_{0} \mid n \geq 0\right\}
$$

and

$$
R_{r e}^{+}(<)=\left\{(n+1) \delta-\alpha_{1}, 2 n \delta+\alpha_{0} \mid n \geq 0\right\}
$$

We then define the total orders on $R_{r e}^{+}(>)$and $R_{r e}^{+}(<)$by

$$
n \delta+\alpha_{1}<(2 n+2) \delta-\alpha_{0}<(n+1) \delta+\alpha_{1}
$$

and

$$
(2 n+2) \delta+\alpha_{0}<(n+1) \delta-\alpha_{1}<2 n \delta+\alpha_{0},
$$

respectively. We also set $R_{i m}^{+}=\{n \delta \mid n \geq 1\}$, the set of positive imaginary roots.

Using the braid group action on $\mathbf{U}$ introduced by Lusztig (see Section 2), we define the real root vector associated with a real root in $R_{r e}^{+}(>)$as follows:

$$
\begin{aligned}
E_{n \delta+\alpha_{1}} & =\left(T_{1}^{-1} T_{0}^{-1}\right)^{n}\left(e_{1}\right), \\
E_{(2 n+2) \delta-\alpha_{0}} & =\left(T_{1}^{-1} T_{0}^{-1}\right)^{n} T_{1}^{-1}\left(e_{0}\right) .
\end{aligned}
$$


We also define the real root vector associated with a real root in $R_{r e}^{+}(<)$as follows:

$$
\begin{aligned}
E_{2 n \delta+\alpha_{0}} & =\left(T_{0} T_{1}\right)^{n}\left(e_{0}\right), \\
E_{(n+1) \delta-\alpha_{1}} & =\left(T_{0} T_{1}\right)^{n} T_{0}\left(e_{1}\right) .
\end{aligned}
$$

Definition 1.1. In the following, each $\mathbf{Z}_{\geq 0}^{(i)}$ is a copy of $\mathbf{Z}_{\geq 0}$ and $E_{\alpha}^{(c)}$ for a real root $\alpha$ and $c \in \mathbf{Z}_{\geq 0}$ denotes the divided power of $E_{\alpha}$ (see Definition $3.2)$, which is known to belong to $\mathbf{U}_{\mathbf{Z}}^{+}$.

(1) For $\mathbf{c}=\left(\mathbf{c}_{i}\right) \in \oplus_{i \in R_{r e}^{+}(>)} \mathbf{Z}_{\geq 0}^{(i)}$, we set $\mathbf{E}_{\mathbf{c}}=E_{\alpha_{1}}^{\left(\mathbf{c}_{\alpha_{1}}\right)} E_{2 \delta-\alpha_{0}}^{\left(\mathbf{c}_{2 \delta-\alpha_{0}}\right)} E_{\delta+\alpha_{1}}^{\left(\mathbf{c}_{\delta+\alpha_{1}}\right)} \cdots$.

(2) We set $B(>)=\left\{\mathbf{E}_{\mathbf{c}} \mid \mathbf{c} \in \oplus_{i \in R_{r e}^{+}(>)} \mathbf{Z}_{\geq 0}^{(i)}\right\}$.

(3) For $\mathbf{c}=\left(\mathbf{c}_{i}\right) \in \oplus_{i \in R_{r e}^{+}(<)} \mathbf{Z}_{\geq 0}^{(i)}$, we set $\mathbf{E}_{\mathbf{c}}=\cdots E_{2 \delta+\alpha_{0}}^{\left(\mathbf{c}_{2 \delta+\alpha_{0}}\right)} E_{\delta-\alpha_{1}}^{\left(\mathbf{c}_{\delta-\alpha_{1}}\right)} E_{\alpha_{0}}^{\left(\mathbf{c}_{\alpha_{0}}\right)}$.

(4) We set $B(<)=\left\{\mathbf{E}_{\mathbf{c}} \mid \mathbf{c} \in \oplus_{i \in R_{r e}^{+}(<)} \mathbf{Z}_{\geq 0}^{(i)}\right\}$.

It is known that both of $B(>)$ and $B(<)$ are linearly independent over $\mathbf{Q}\left(q_{1}\right)[\mathrm{L}, 40.2 .1]$. Let $\mathbf{U}^{+}(>)$(resp. $\mathbf{U}^{+}(<)$) be the vector subspace of $\mathbf{U}^{+}$ over $\mathbf{Q}\left(q_{1}\right)$ with basis $B(>)$ (resp. $\left.B(<)\right)$ : it is known that they are algebras over $\mathbf{Q}\left(q_{1}\right)$.

We define the imaginary root vector $\tilde{\psi}_{n}$ associated with an imaginary root $n \delta \in R_{i m}^{+}$as follows:

$$
\begin{aligned}
\tilde{\psi}_{n} & =\left[E_{\delta-\alpha_{1}}, E_{(n-1) \delta+\alpha_{1}}\right]_{q^{-1}} \\
& :=E_{\delta-\alpha_{1}} E_{(n-1) \delta+\alpha_{1}}-q^{-1} E_{(n-1) \delta+\alpha_{1}} E_{\delta-\alpha_{1}} .
\end{aligned}
$$

We prove that they are mutually commutative and algebraically independent over $\mathbf{Q}\left(q_{1}\right)$. Let $\mathbf{U}^{+}(0)$ be the subalgebra of $\mathbf{U}^{+}$generated by them.

We prove that the $\mathbf{Q}\left(q_{1}\right)$-linear map $\mathbf{U}^{+}(>) \otimes \mathbf{U}^{+}(0) \otimes \mathbf{U}^{+}(<) \longrightarrow \mathbf{U}^{+}$ given by multiplication is an isomorphism: this solves the problem raised in Lusztig's book [L, 40.2.5] in the $A_{2}^{(2)}$-case. Namely, we prove the following.

Theorem 1.2. For $\mathbf{c}=\left(\mathbf{c}_{i}\right) \in \oplus_{i \in \mathbf{Z}_{\geq 1}} \mathbf{Z}_{\geq 0}^{(i)}$, we set $\mathbf{E}_{\mathbf{c}}^{\prime}=\tilde{\psi}_{1}^{\mathbf{c}_{1}} \tilde{\psi}_{2}^{\mathbf{c}_{2}} \tilde{\psi}_{3}^{\mathbf{c}_{3}} \cdots$ Then, the following is a $\mathbf{Q}\left(q_{1}\right)$-basis of $\mathbf{U}^{+}$:

$$
\left\{\mathbf{E}_{\mathbf{c}_{+}} \mathbf{E}_{\mathbf{c}_{\mathbf{0}}}^{\prime} \mathbf{E}_{\mathbf{c}_{-}} \mid \mathbf{c}_{+} \in \oplus_{i \in R_{r e}^{+}(>)} \mathbf{Z}_{\geq 0}^{(i)}, \mathbf{c}_{\mathbf{0}} \in \oplus_{n \geq 1} \mathbf{Z}_{\geq 0}^{(n)}, \mathbf{c}_{-} \in \oplus_{i \in R_{r e}^{+}(<)} \mathbf{Z}_{\geq 0}^{(i)}\right\} .
$$

Modifying this basis, we construct a $\mathbf{Z}\left[q_{1}, q_{1}^{-1}\right]$-basis of $\mathbf{U}_{\mathbf{Z}}^{+}$. Let $\mathbf{U}_{\mathbf{Z}}^{+}(>)$ (resp. $\mathbf{U}_{\mathbf{Z}}^{+}(<)$) be the free $\mathbf{Z}\left[q_{1}, q_{1}^{-1}\right]$-submodule of $\mathbf{U}_{\mathbf{Z}}^{+}$with basis $B(>$ ) (resp. 
$B(<))$. It turns out that they are algebras over $\mathbf{Z}\left[q_{1}, q_{1}^{-1}\right][\mathrm{BCP}$, Proposition 2.3]. Now, if we choose $\mathbf{U}_{\mathbf{Z}}^{+}(0)$ as the $\mathbf{Z}\left[q_{1}, q_{1}^{-1}\right]$-subalgebra of $\mathbf{U}_{\mathbf{Z}}^{+}$generated by the $\tilde{\psi}_{n}$ 's, then the $\mathbf{Z}\left[q_{1}, q_{1}^{-1}\right]$-linear map $\mathbf{U}_{\mathbf{Z}}^{+}(>) \otimes_{\mathbf{Z}\left[q_{1}, q_{1}^{-1}\right]} \mathbf{U}_{\mathbf{Z}}^{+}(0) \otimes_{\mathbf{Z}\left[q_{1}, q_{1}^{-1}\right]}$ $\mathbf{U}_{\mathbf{Z}}^{+}(<) \longrightarrow \mathbf{U}_{\mathbf{Z}}^{+}$given by multiplication is injective, but it is not surjective. Therefore, in order to construct a $\mathbf{Z}\left[q_{1}, q_{1}^{-1}\right]$-basis of $\mathbf{U}_{\mathbf{Z}}^{+}$, we have to find an appropriate definition of $\mathbf{U}_{\mathbf{Z}}^{+}(0) \subset \mathbf{U}_{\mathbf{Z}}^{+} \cap \mathbf{U}^{+}(0)$ so that the above multiplication morphism is an isomorphism. Instead of the $\tilde{\psi}_{n}$, we introduce the new imaginary root vectors $P_{n} \in \mathbf{U}^{+}(0)$ as follows: we set $P_{0}=1$ and

$$
P_{n}=[2 n]_{1}^{-1} \sum_{k=0}^{n-1} P_{k} \tilde{\psi}_{n-k} q^{-k} \quad \text { for } n \geq 1 \text {. }
$$

We prove that the $P_{n}$ belong to $\mathbf{U}_{\mathbf{Z}}^{+}$. This statement is not at all evident, whereas $\tilde{\psi}_{n} \in \mathbf{U}_{\mathbf{Z}}^{+}$is evident. Then, we define $\mathbf{U}_{\mathbf{Z}}^{+}(0)$ as the $\mathbf{Z}\left[q_{1}, q_{1}^{-1}\right]$ subalgebra of $\mathbf{U}_{\mathbf{Z}}^{+}$generated by the $P_{n}$ 's and prove that the $\mathbf{Z}\left[q_{1}, q_{1}^{-1}\right]$-linear $\operatorname{map} \mathbf{U}_{\mathbf{Z}}^{+}(>) \otimes_{\mathbf{Z}\left[q_{1}, q_{1}^{-1}\right]} \mathbf{U}_{\mathbf{Z}}^{+}(0) \otimes_{\mathbf{Z}\left[q_{1}, q_{1}^{-1}\right]} \mathbf{U}_{\mathbf{Z}}^{+}(<) \longrightarrow \mathbf{U}_{\mathbf{Z}}^{+}$is an isomorphism. In this way, we obtain

Theorem 1.3. For $\mathbf{c}=\left(\mathbf{c}_{i}\right) \in \oplus_{i \in \mathbf{Z}_{\geq 1}} \mathbf{Z}_{\geq 0}^{(i)}$, we set $\mathbf{E}_{\mathbf{c}}=P_{1}^{\mathbf{c}_{1}} P_{2}^{\mathbf{c}_{2}} P_{3}^{\mathbf{c}_{3}} \cdots$. Then, the following is a $\mathbf{Z}\left[q_{1}, q_{1}^{-1}\right]$-basis of $\mathbf{U}_{\mathbf{Z}}^{+}$:

$$
\left\{\mathbf{E}_{\mathbf{c}_{+}} \mathbf{E}_{\mathbf{c}_{\mathbf{0}}} \mathbf{E}_{\mathbf{c}_{-}} \mid \mathbf{c}_{+} \in \oplus_{i \in R_{r e}^{+}(>)} \mathbf{Z}_{\geq 0}^{(i)}, \mathbf{c}_{\mathbf{0}} \in \oplus_{n \geq 1} \mathbf{Z}_{\geq 0}^{(n)}, \mathbf{c}_{-} \in \oplus_{i \in R_{r e}^{+}(<)} \mathbf{Z}_{\geq 0}^{(i)}\right\} .
$$

However, this basis does not give an integral crystal basis: we need a further modification. For a partition $\lambda$, we define $\mathbf{S}_{\lambda} \in \mathbf{U}_{\mathbf{Z}}^{+}(0)$ from the $P_{n}$ in the same way as the Schur functions are defined from the complete symmetric functions; namely, we set

$$
\mathbf{S}_{\lambda}=\operatorname{det}\left(P_{\lambda_{i}-i+j}\right)_{i, j \geq 1} \quad \text { for } \quad \lambda=\left(\lambda_{1} \geq \lambda_{2} \geq \cdots\right)
$$

where we understand that $P_{n}=0$ for $n \leq-1$. Then, it follows that the $\mathbf{S}_{\lambda}$ are quasi-orthonormal with respect to the inner product on $\mathbf{U}^{+}$introduced by Drinfeld, that is,

$$
\left(\mathbf{S}_{\lambda}, \mathbf{S}_{\mu}\right) \equiv \delta_{\lambda, \mu} \quad \bmod q_{1}^{-1} \mathbf{A} .
$$

Here, $\mathbf{A}=\mathbf{Q}\left(q_{1}\right) \cap \mathbf{Z}\left[\left[q_{1}^{-1}\right]\right] \subset \mathbf{Q}\left(\left(q_{1}^{-1}\right)\right)$. Therefore, the following is another $\mathbf{Z}\left[q_{1}, q_{1}^{-1}\right]$-basis of $\mathbf{U}_{\mathbf{Z}}^{+}$:

$\left\{\mathbf{E}_{\mathbf{c}_{+}} \mathbf{S}_{\lambda} \mathbf{E}_{\mathbf{c}_{-}} \mid \mathbf{c}_{+} \in \oplus_{i \in R_{r e}^{+}(>)} \mathbf{Z}_{\geq 0}^{(i)}, \lambda\right.$ is a partition, $\left.\mathbf{c}_{-} \in \oplus_{i \in R_{r e}^{+}(<)} \mathbf{Z}_{\geq 0}^{(i)}\right\}$,

which is denoted by $B$. In view of [L, 40.2.4], we see that $B$ is quasi-orthonormal with respect to the inner product. Hence, by the same argument in $[\mathrm{BCP}]$, we obtain 
Theorem 1.4. $\quad B$ is an integral crystal bases of $\mathbf{U}^{+}$.

The contents of this paper are as follows.

In Section 2, we fix notations. Automorphism $T_{\varpi_{1}}=T_{0} T_{1}$ and antiautomorphism $T_{1}^{-1} *$ of $\mathbf{U}$ play an important role in this paper.

In Section 3, we introduce the root vectors as above and study their commutation relations. We prove that the imaginary root vectors mutually commute and are invariant under $T_{\varpi_{1}}=T_{0} T_{1}$ and $T_{1}^{-1} *$. The key step in this section is to express the real root vectors recursively using brackets (Corollary 3.10), which the author learned from [KhT, 8.2].

In Section 4, we introduce the subspaces $\mathbf{U}^{+}(>), \mathbf{U}^{+}(<), \mathbf{U}^{+}(0)$ of $\mathbf{U}^{+}$as above, and prove that the $\mathbf{Q}\left(q_{1}\right)$-linear map $\mathbf{U}^{+}(>) \otimes \mathbf{U}^{+}(0) \otimes \mathbf{U}^{+}(<) \longrightarrow \mathbf{U}^{+}$ given by multiplication is surjective: the proof of its injectivity with the help of $[\mathrm{L}, 40.1 .2]$ is postponed until Section 6 . We also introduce the $P_{n}$ in this section.

In Section 5, we study the coproducts of the real root vectors.

In Section 6, we calculate the coproducts and the inner products of the imaginary root vectors and introduce the $\mathbf{S}_{\lambda}$ : the results in this section are used to construct an integral crystal basis in Section 8. As a by-product, the algebraically independence of the imaginary root vectors (the $\tilde{\psi}_{n}$ 's or the $P_{n}$ 's) is proved; thus, the monomials of the imaginary root vectors (the $\tilde{\psi}_{n}$ 's or the $P_{n}$ 's) form a basis of $\mathbf{U}^{+}(0)$ and we obtain PBW bases of $\mathbf{U}^{+}$.

Section 7 is the preparation for the next section.

In Section 8, we give the commutation relation between $E_{\alpha_{0}}^{(s)}$ and $E_{\alpha_{1}}^{(r)}$. Using it, we prove that the $P_{n}$ belong to $\mathbf{U}_{\mathbf{Z}}^{+}$and construct an integral PBW basis of $\mathbf{U}^{+}$. Then, we obtain an integral crystal basis of $\mathbf{U}^{+}$.

In Appendix A, we give certain commutation relation between the real root vectors that is used in Section 4.

In Appendix B, we discuss the connection between our root vectors and the Drinfeld generators.

After writing up the main part of this paper, the author learned the existence of [Da2], in which (non-integral) PBW bases of the twisted quantum affine algebras are constructed.

The author is grateful to Professor Masaki Kashiwara for valuable comments and useful discussions on this work.

\section{$\S 2 . \quad$ Notation}

Let $X, Y$ be the finitely generated free $\mathbf{Z}$-modules with a perfect pairing $\langle\rangle:, Y \times X \longrightarrow \mathbf{Z}$. Let $\alpha_{0} \in X$ be the long simple root and $\alpha_{1} \in X$ the 
short simple root of type $A_{2}^{(2)}$ : we assume that they are linearly independent. We set $I=\{0,1\}$. For $i \in I$, let $h_{i} \in Y$ be the simple coroots: we assume that they are linearly independent. Let $Q=\mathbf{Z} \alpha_{0} \oplus \mathbf{Z} \alpha_{1} \subset X$ be the root lattice and let $Q^{+}=\mathbf{Z}_{\geq 0} \alpha_{0} \oplus \mathbf{Z}_{\geq 0} \alpha_{1}, Q^{-}=-Q^{+}$. Let $($, ) be the $\mathbf{Z}$-valued symmetric bilinear form on $X$ such that

$$
\left(\alpha_{0}, \alpha_{0}\right)=4, \quad\left(\alpha_{0}, \alpha_{1}\right)=-2, \quad\left(\alpha_{1}, \alpha_{1}\right)=1,
$$

so that the Cartan matrix is given by

$$
\left(\left\langle h_{i}, \alpha_{j}\right\rangle\right)_{i, j}=\left(\begin{array}{ll}
a_{00} & a_{01} \\
a_{10} & a_{11}
\end{array}\right)=\left(\begin{array}{cc}
2 & -1 \\
-4 & 2
\end{array}\right) .
$$

Let $\delta=\alpha_{0}+2 \alpha_{1} \in X$ be the smallest positive imaginary root. Note that $Q=\mathbf{Z} \delta \oplus \mathbf{Z} \alpha_{1}$. We set

$$
R_{r e}^{+}(>)=\left\{n \delta+\alpha_{1},(2 n+2) \delta-\alpha_{0} \mid n \geq 0\right\}
$$

and define the total order on it by

$$
n \delta+\alpha_{1}<(2 n+2) \delta-\alpha_{0}<(n+1) \delta+\alpha_{1}
$$

for $n \geq 0$; we set

$$
R_{r e}^{+}(<)=\left\{(n+1) \delta-\alpha_{1}, 2 n \delta+\alpha_{0} \mid n \geq 0\right\}
$$

and define the total order on it by

$$
(2 n+2) \delta+\alpha_{0}<(n+1) \delta-\alpha_{1}<2 n \delta+\alpha_{0}
$$

for $n \geq 0$; we also set $R_{i m}^{+}=\{n \delta \mid n \geq 1\}$. Then, $R_{r e}^{+}=R_{r e}^{+}(>) \sqcup R_{r e}^{+}(<)$is the set of positive real roots; $R^{+}=R_{r e}^{+} \sqcup R_{i m}^{+}$is the set of positive roots; and $R=R^{+} \sqcup\left(-R^{+}\right)$is the set of roots.

We set $q_{0}=q^{2}, q_{1}=q^{1 / 2}$. For $i \in I, k \in \mathbf{Z}, n \in \mathbf{Z}_{\geq 1}$, we set $[k]_{i}=\left(q_{i}^{k}-\right.$ $\left.q_{i}^{-k}\right) /\left(q_{i}-q_{i}^{-1}\right),[n]_{i} !=\prod_{p=1}^{l}[p]_{i},[0]_{i} !=1,[k]=\left(q^{k}-q^{-k}\right) /\left(q-q^{-1}\right),[n] !=$ $\prod_{p=1}^{l}[p],[0] !=1$. For $i \in I, n, m \geq 0$, we set $\left[\begin{array}{c}n+m \\ m\end{array}\right]_{i}=\left([n+m]_{i} !\right) /\left([n]_{i} ![m]_{i} !\right)$, which belong to $\mathbf{Z}\left[q_{i}, q_{i}^{-1}\right]$.

Definition 2.1. $\quad$ Let $\mathbf{U}$ be the quantum affine algebra of type $A_{2}^{(2)}$, which is the $\mathbf{Q}\left(q_{1}\right)$-algebra generated by

$$
\left\{q^{h}, e_{i}, f_{i} \mid h \in 2^{-1} Y, i \in I\right\}
$$


with the following defining relations:

$$
\begin{aligned}
& q^{0}=1, q^{h} q^{h^{\prime}}=q^{h+h^{\prime}} \text { for } h, h^{\prime} \in 2^{-1} Y, \\
& q^{h} e_{i} q^{-h}=q^{\left\langle h, \alpha_{i}\right\rangle} e_{i} \text { for } h \in 2^{-1} Y, i \in I, \\
& q^{h} f_{i} q^{-h}=q^{-\left\langle h, \alpha_{i}\right\rangle} f_{i} \text { for } h \in 2^{-1} Y, i \in I, \\
& {\left[e_{i}, f_{j}\right]=\delta_{i j} \frac{k_{i}-k_{i}^{-1}}{q_{i}-q_{i}^{-1}} \text { for } i, j \in I,} \\
& \sum_{k=0}^{1-a_{i j}}(-1)^{k} e_{i}^{(k)} e_{j} e_{i}^{\left(1-a_{i j}-k\right)}=0 \text { for } i, j \in I \text { with } i \neq j, \\
& \sum_{k=0}^{1-a_{i j}}(-1)^{k} f_{i}^{(k)} f_{j} f_{i}^{\left(1-a_{i j}-k\right)}=0 \text { for } i, j \in I \text { with } i \neq j .
\end{aligned}
$$

Here, we set $k_{0}=q^{2 h_{0}}, k_{1}=q^{2^{-1} h_{1}}$ and $e_{i}^{(k)}=e_{i}^{k} /[k]_{i} !, f_{i}^{(k)}=f_{i}^{k} /[k]_{i}$ ! for $i \in I, k \geq 0$.

Remark 2.2. We have $k_{i} e_{j} k_{i}^{-1}=q^{\left(\alpha_{i}, \alpha_{j}\right)} e_{j}, k_{i} f_{j} k_{i}^{-1}=q^{-\left(\alpha_{i}, \alpha_{j}\right)} f_{j}$ for $i, j \in I$.

Definition 2.3. For $\mu=n \alpha_{0}+m \alpha_{1} \in Q$, we set $k_{\mu}=k_{0}^{n} k_{1}^{m}$. We also set $c=k_{\delta}=k_{0} k_{1}^{2}=q^{2 h_{0}+h_{1}}$, which is a central element of $\mathbf{U}$.

Definition 2.4. (1) Let $\mathbf{U}^{0}$ be the $\mathbf{Q}\left(q_{1}\right)$-algebra generated by $q^{h}$ for $h \in 2^{-1} Y$ with the defining relations (U1).

(2) Let $\mathbf{U}^{+}$be the $\mathbf{Q}\left(q_{1}\right)$-algebra generated by $e_{i}$ for $i \in I$ with the defining relations (U5).

(3) Let $\mathbf{U}^{-}$be the $\mathbf{Q}\left(q_{1}\right)$-algebra generated by $f_{i}$ for $i \in I$ with the defining relations (U6).

Then, $\left\{q^{h} \in \mathbf{U}^{0} \mid h \in 2^{-1} Y\right\}$ is a $\mathbf{Q}\left(q_{1}\right)$-basis of $\mathbf{U}^{0}$.

Proposition 2.5 [L, 3.2.5]. The $\mathbf{Q}\left(q_{1}\right)$-linear map $\mathbf{U}^{-} \otimes \mathbf{U}^{0} \otimes \mathbf{U}^{+} \rightarrow \mathbf{U}$ given by multiplication is an isomorphism.

Hence, $\mathbf{U}^{0}, \mathbf{U}^{+}, \mathbf{U}^{-}$can be considered as the subalgebras of $\mathbf{U}$.

If a nonzero element $x$ of $\mathbf{U}^{+}$has a homogeneous expression in terms of $e_{i}$ $(i \in I)$, then the indices $i_{1}, \ldots, i_{k}\left(i_{j} \in I, k \geq 0\right)$ appearing in it are uniquely determined up to permutation, and we say that $x$ is homogeneous of weight $\sum_{j=1}^{k} \alpha_{i_{j}}$, which is denoted by $|x|$. We apply the similar definition for $\mathbf{U}^{-}$. 
Definition 2.6. For $\alpha \in Q^{+}$, we set

$$
\mathbf{U}_{\alpha}^{+}=\left\{x \in \mathbf{U}^{+} \mid x=0 \text { or } x \text { is homogeneous of weight } \alpha\right\} .
$$

We also set

$$
\mathbf{U}^{+, h}=\left\{x \in \mathbf{U}^{+} \mid x=0 \text { or } x \text { is homogeneous }\right\},
$$

which is closed under multiplication. For $\alpha \in Q^{-}$, we define $\mathbf{U}_{\alpha}^{-}$similarly.

We have $\mathbf{U}^{+, h}=\sqcup_{\alpha \in Q^{+}}\left(\mathbf{U}_{\alpha}^{+} \backslash\{0\}\right) \sqcup\{0\}$.

Definition 2.7. For $\nu \in Q$, we set $\mathbf{U}_{\nu}=\oplus_{\lambda \in Q^{-}, \mu \in Q^{+} ; \lambda+\mu=\nu} \mathbf{U}_{\lambda}^{-} \mathbf{U}^{0} \mathbf{U}_{\mu}^{+}$.

Definition 2.8. For a subset $A$ of $\mathbf{U}^{+}$and for $i \in \mathbf{Z}$, we write

$$
\begin{aligned}
A^{h} & =A \cap \mathbf{U}^{+, h}, \\
A_{\leq i} & =A \cap \oplus_{\alpha=n \delta+r \alpha_{1} \in Q^{+} ; r \leq i} \mathbf{U}_{\alpha}^{+} .
\end{aligned}
$$

Definition 2.9. We define the function $\mathrm{h}$ from $\mathbf{U}^{+, h} \backslash\{0\}$ to $\mathbf{Z}$ by $\mathrm{h}(x)$ $=r$ for $x \in \mathbf{U}_{n \delta+r \alpha_{1}}^{+} \backslash\{0\}$. We call $\mathrm{h}(x)$ the height of $x$. We also define the function i from $\mathbf{U}^{+, h} \backslash\{0\}$ to $\mathbf{Z}_{\geq 0}$ by i $(x)=n$ for $x \in \mathbf{U}_{n \delta+r \alpha_{1}}^{+} \backslash\{0\}$.

It is known that $\mathbf{U}^{+} \backslash\{0\}$ is closed under multiplication; thus, we have $\mathrm{h}(x y)=\mathrm{h}(x)+\mathrm{h}(y)$ and $\mathrm{i}(x y)=\mathrm{i}(x)+\mathrm{i}(y)$ for $x, y \in \mathbf{U}^{+, h} \backslash\{0\}$.

Definition 2.10. For elements $x, y$ of a $\mathbf{Q}\left(q_{1}\right)$-algebra and for $v \in$ $\mathbf{Q}\left(q_{1}\right)^{\times}$, we set

$$
[x, y]_{v}=x y-v y x .
$$

When $v=1$, we omit the suffix $v$.

Lemma 2.11. Let $x, y, z$ be elements of a $\mathbf{Q}\left(q_{1}\right)$-algebra and let $\alpha, \beta \in$ $\mathbf{Q}\left(q_{1}\right)^{\times}$. Then,

(1) $[x, y z]_{\alpha}=[x, y]_{\beta} z+y[x, z]_{\alpha / \beta} \beta$,

(2) $[x y, z]_{\alpha}=x[y, z]_{\beta}+[x, z]_{\alpha / \beta} y \beta$.

Proof. This is clear.

Definition 2.12. Let $\mathbf{U}_{\mathbf{Z}}$ be the $\mathbf{Z}\left[q_{1}, q_{1}^{-1}\right]$-subalgebra of $\mathbf{U}$ generated by $e_{i}^{(r)}, f_{i}^{(r)}, k_{i}, k_{i}^{-1}$ for $i \in I, r \geq 0$. 
Definition 2.13. For $i \in I, m \in \mathbf{Z}, r \in \mathbf{Z}_{\geq 0}$, we set

$$
\left[\begin{array}{c}
k_{i}, m \\
r
\end{array}\right]_{i}=\prod_{s=1}^{r} \frac{k_{i} q_{i}^{m-s+1}-k_{i}^{-1} q_{i}^{-m+s-1}}{q_{i}^{s}-q_{i}^{-s}} .
$$

We understand that $\left[\begin{array}{c}k_{i}, m \\ 0\end{array}\right]_{i}=1$. Then, they belong to $\mathbf{U}_{\mathbf{Z}}$, which follows from Lemma 2.14 below.

Lemma 2.14 [L, 3.1.9]. Let $n, m \geq 0, i \in I$. Then,

$$
e_{i}^{(n)} f_{i}^{(m)}=\sum_{t=0}^{\min (n, m)} f_{i}^{(m-t)}\left[\begin{array}{c}
k_{i}, 2 t-n-m \\
t
\end{array}\right]_{i} e_{i}^{(n-t)} .
$$

Corollary 2.15. Let $r \geq 1, i \in I$. Then,

$$
\left[e_{i}^{(r)}, f_{i}\right]=e_{i}^{(r-1)} \frac{q_{i}^{r-1} k_{i}-q_{i}^{1-r} k_{i}^{-1}}{q_{i}-q_{i}^{-1}} .
$$

Proof. This follows from Lemma 2.14 with $m=1$.

Lemma 2.16. Let $i, j \in I, m \in \mathbf{Z}, r \in \mathbf{Z}_{\geq 0}$. Then,

(1) $e_{j}\left[\begin{array}{c}k_{i}, m \\ t\end{array}\right]_{i}=\left[\begin{array}{c}k_{i}, m-a_{i j} \\ t\end{array}\right]_{i} e_{j}$,

(2) $\left[\begin{array}{c}k_{i}, m \\ t\end{array}\right]_{i} f_{j}=f_{j}\left[\begin{array}{c}k_{i}, m-a_{i j} \\ t\end{array}\right]_{i}$.

Proof. This is directly checked.

Definition 2.17. (1) Let $\mathbf{U}_{\mathbf{Z}}^{0}$ be the $\mathbf{Z}\left[q_{1}, q_{1}^{-1}\right]$-subalgebra of $\mathbf{U}$ generated by $k_{i}, k_{i}^{-1},\left[\begin{array}{c}k_{i}, m \\ r\end{array}\right]_{i}$ for $i \in I, m \in \mathbf{Z}, r \in \mathbf{Z}_{\geq 1}$.

(2) Let $\mathbf{U}_{\mathbf{Z}}^{+}$be the $\mathbf{Z}\left[q_{1}, q_{1}^{-1}\right]$-subalgebra of $\mathbf{U}$ generated by $e_{i}^{(r)}$ for $i \in I, r \geq 0$.

(3) Let $\mathbf{U}_{\mathbf{Z}}^{-}$be the $\mathbf{Z}\left[q_{1}, q_{1}^{-1}\right]$-subalgebra of $\mathbf{U}$ generated by $f_{i}^{(r)}$ for $i \in I, r \geq 0$.

We have $\mathbf{U}_{\mathbf{Z}}^{+}=\oplus_{\alpha \in Q^{+}}\left(\mathbf{U}_{\mathbf{Z}}^{+} \cap \mathbf{U}_{\alpha}^{+}\right)$and $\mathbf{U}_{\mathbf{Z}}^{-}=\oplus_{\alpha \in Q^{-}}\left(\mathbf{U}_{\mathbf{Z}}^{-} \cap \mathbf{U}_{\alpha}^{-}\right)$, since the generators of $\mathbf{U}_{\mathbf{Z}}^{+}$and $\mathbf{U}_{\mathbf{Z}}^{-}$are homogeneous.

Lemma 2.18 [L1, 4.5]. The following set $B_{0}$ is a $\mathbf{Z}\left[q_{1}, q_{1}^{-1}\right]$-basis of $\mathbf{U}_{\mathbf{Z}}^{0}$ :

$$
\left\{k_{0}^{a} k_{1}^{b}\left[\begin{array}{c}
k_{0}, 0 \\
r
\end{array}\right]_{0}\left[\begin{array}{c}
k_{1}, 0 \\
s
\end{array}\right]_{1} \mid a, b \in\{0,1\}, r, s \geq 0\right\} .
$$


Proposition 2.19. The $\mathbf{Z}\left[q_{1}, q_{1}^{-1}\right]$-linear map $\quad \mathbf{U}_{\mathbf{Z}}^{-} \quad \otimes_{\mathbf{Z}\left[q_{1}, q_{1}^{-1}\right]} \mathbf{U}_{\mathbf{Z}}^{0}$ $\otimes_{\mathbf{Z}\left[q_{1}, q_{1}^{-1}\right]} \mathbf{U}_{\mathbf{Z}}^{+} \rightarrow \mathbf{U}_{\mathbf{Z}}$ given by multiplication is an isomorphism.

Proof. Note that $\mathbf{U}_{\mathbf{Z}}^{+}$and $\mathbf{U}_{\mathbf{Z}}^{-}$are free $\mathbf{Z}\left[q_{1}, q_{1}^{-1}\right]$-modules by the existence of the canonical bases; thus, the injectivity follows from Proposition 2.5. The surjectivity follows from Lemmas 2.14 and 2.16 .

Noting that $\mathbf{U}_{\mathbf{Z}}^{0}, \mathbf{U}_{\mathbf{Z}}^{+}, \mathbf{U}_{\mathbf{Z}}^{-}$are free $\mathbf{Z}\left[q_{1}, q_{1}^{-1}\right]$-modules and using Proposition 2.19, we have $\mathbf{U}_{\mathbf{Z}}^{+}=\mathbf{U}_{\mathbf{Z}} \cap \mathbf{U}^{+}$.

Definition 2.20 [K1, 3.4.1], [L, 1.2.13]. Let $i \in I$. We define the $\mathbf{Q}\left(q_{1}\right)$ linear maps $r_{i}$ and ${ }_{i} r$ from $\mathbf{U}^{+}$to itself by $r_{i}(1)={ }_{i} r(1)=0, r_{i}\left(e_{j}\right)={ }_{i} r\left(e_{j}\right)=$ $\delta_{i j}$, and

(1) $r_{i}(x y)=q^{\left(\alpha_{i},|y|\right)} r_{i}(x) y+x r_{i}(y)$ for $x, y \in \mathbf{U}^{+, h}$,

(2) ${ }_{i} r(x y)={ }_{i} r(x) y+q^{\left(\alpha_{i},|x|\right)} x{ }_{i} r(y)$ for $x, y \in \mathbf{U}^{+, h}$.

Lemma 2.21 [K1, 3.4.2], [L, 1.2.15]. Let $\alpha \in Q^{+} \backslash\{0\}, x \in \mathbf{U}_{\alpha}^{+, h}$.

(1) If $r_{i}(x)=0$ for all $i \in I$, then $x=0$.

(2) If ${ }_{i} r(x)=0$ for all $i \in I$, then $x=0$.

Lemma 2.22 [K1, 3.4.1], [L, 3.1.6]. Let $x \in \mathbf{U}^{+, h}, i \in I$. Then,

$$
\left[x, f_{i}\right]=\frac{r_{i}(x) k_{i}-k_{i}^{-1}{ }_{i} r(x)}{q_{i}-q_{i}^{-1}} .
$$

Let us recall the braid group action on $\mathbf{U}$ introduced by Lusztig. Recall that the braid group of type $A_{2}^{(2)}$ is the free group generated by $T_{0}$ and $T_{1}$.

Definition 2.23 [L, 37.1.3]. For $i \in I$, the following gives the automorphisms $T_{i}$ of $\mathbf{U}$ :

$$
\begin{aligned}
T_{i}\left(q^{h}\right) & =q^{h-\left\langle h, \alpha_{i}\right\rangle h_{i}} \text { for } h \in 2^{-1} Y, \\
T_{i}\left(e_{i}\right) & =-f_{i} k_{i}, T_{i}\left(f_{i}\right)=-k_{i}^{-1} e_{i}, \\
T_{i}\left(e_{j}\right) & =\sum_{r=0}^{-a_{i j}}(-1)^{r} q_{i}^{-r} e_{i}^{\left(-a_{i j}-r\right)} e_{j} e_{i}^{(r)} \text { for } j \in I \text { with } j \neq i, \\
T_{i}\left(f_{j}\right) & =\sum_{r=0}^{-a_{i j}}(-1)^{r} q_{i}^{r} f_{i}^{(r)} f_{j} f_{i}^{\left(-a_{i j}-r\right)} \text { for } j \in I \text { with } j \neq i, \\
T_{i}^{-1}\left(q^{h}\right) & =q^{h-\left\langle h, \alpha_{i}\right\rangle h_{i}} \text { for } h \in 2^{-1} Y,
\end{aligned}
$$




$$
\begin{aligned}
T_{i}^{-1}\left(e_{i}\right) & =-k_{i}^{-1} f_{i}, T_{i}^{-1}\left(f_{i}\right)=-e_{i} k_{i}, \\
T_{i}^{-1}\left(e_{j}\right) & =\sum_{r=0}^{-a_{i j}}(-1)^{r} q_{i}^{-r} e_{i}^{(r)} e_{j} e_{i}^{\left(-a_{i j}-r\right)} \text { for } j \in I \text { with } j \neq i, \\
T_{i}^{-1}\left(f_{j}\right) & =\sum_{r=0}^{-a_{i j}}(-1)^{r} q_{i}^{r} f_{i}^{\left(-a_{i j}-r\right)} f_{j} f_{i}^{(r)} \text { for } j \in I \text { with } j \neq i .
\end{aligned}
$$

Moreover, $\mathbf{U}_{\mathbf{Z}}$ is closed under the braid group action.

We have $T_{i}\left(k_{j}\right)=T_{i}^{-1}\left(k_{j}\right)=k_{i}^{-a_{i j}} k_{j}$ for $i, j \in I$.

Remark 2.24. In [L], $T_{i}$ and $T_{i}^{-1}$ are denoted by $T_{i, 1}^{\prime \prime}$ and $T_{i,-1}^{\prime}$ respectively.

Definition 2.25. We set $T_{\varpi_{1}}=T_{0} T_{1}$.

We have $T_{\varpi_{1}}(c)=c, T_{\varpi_{1}}\left(k_{1}\right)=c^{-1} k_{1}, T_{\varpi_{1}}\left(k_{0}\right)=c^{2} k_{0}$.

Definition 2.26. Let $\Delta$ be the coproduct of $\mathbf{U}$ given by

$$
\begin{aligned}
\Delta\left(q^{h}\right) & =q^{h} \otimes q^{h}, \\
\Delta\left(e_{i}\right) & =e_{i} \otimes 1+k_{i} \otimes e_{i}, \\
\Delta\left(f_{i}\right) & =f_{i} \otimes k_{i}^{-1}+1 \otimes f_{i}
\end{aligned}
$$

for $h \in 2^{-1} Y, i \in I$.

Definition 2.27. Let - be the Q-linear involution of $\mathbf{U}$ given by

$$
\overline{q_{1}}=q_{1}^{-1}, \overline{q^{h}}=q^{-h}, \overline{e_{i}}=e_{i}, \overline{f_{i}}=f_{i} \text { for } h \in 2^{-1} Y, i \in I .
$$

Definition 2.28. Let $*$ be the $\mathbf{Q}\left(q_{1}\right)$-linear anti-involution of $\mathbf{U}$ given by

$$
*\left(q^{h}\right)=q^{-h}, *\left(e_{i}\right)=e_{i}, *\left(f_{i}\right)=f_{i} \text { for } h \in 2^{-1} Y, i \in I .
$$

We have $* T_{i}=T_{i}^{-1} *$ for $i \in I$.

Definition 2.29. Let $\Omega$ be the $\mathbf{Q}$-linear anti-involution of $\mathbf{U}$ given by

$$
\Omega\left(q_{1}\right)=q_{1}^{-1}, \Omega\left(q^{h}\right)=q^{-h}, \Omega\left(e_{i}\right)=f_{i}, \Omega\left(f_{i}\right)=e_{i} \text { for } h \in 2^{-1} Y, i \in I .
$$

We have $\Omega T_{i}=T_{i} \Omega$ for $i \in I$. We also have $\Omega\left(\mathbf{U}^{+}\right)=\mathbf{U}^{-}$and $\Omega\left(\mathbf{U}_{\mathbf{Z}}^{+}\right)=$ $\mathbf{U}_{\mathbf{Z}}^{-}$. 
Remark 2.30. Given a $\mathbf{Q}\left(q_{1}\right)$-basis $B$ of $\mathbf{U}^{+}$, we obtain a $\mathbf{Q}\left(q_{1}\right)$-basis of $\mathbf{U}$ :

$$
\left\{\Omega(b) q^{h} b^{\prime} \mid b, b^{\prime} \in B, h \in 2^{-1} Y\right\}
$$

Given a $\mathbf{Z}\left[q_{1}, q_{1}^{-1}\right]$-basis $B$ of $\mathbf{U}_{\mathbf{Z}}^{+}$, by Lemma 2.18 , we obtain a $\mathbf{Z}\left[q_{1}, q_{1}^{-1}\right]$-basis of $\mathbf{U}_{\mathbf{Z}}$ :

$$
\left\{\Omega(b) x b^{\prime} \mid x \in B_{0}, b, b^{\prime} \in B\right\}
$$

Remark 2.31. Let us set the general notation used in this paper.

(1) Algebras are always assumed to be associative with 1 and any morphism of algebras is assumed to preserve 1 .

(2) For a statement $P$, the symbol $\theta(P)$ means 1 if $P$ is true and 0 otherwise.

(3) For a set $S$ and for $i, j \in S$, we write $\delta_{i j}=\theta(i=j)$.

(4) Any summation considered in $\mathbf{U}$ is taken over a finite set.

(5) For $\mathbf{Q}\left(q_{1}\right)$-vector subspaces $A, B$ of $\mathbf{U}$, we denote by $A \otimes B$ the tensor product of $A$ and $B$ over $\mathbf{Q}\left(q_{1}\right)$.

(6) For subsets $A, B$ of $\mathbf{U}$, we denote by $A B$ the $\mathbf{Z}$-submodule of $\mathbf{U}$ generated by $\{a b \mid a \in A, b \in B\}$; thus, for a subring $C$ of $\mathbf{Q}\left(q_{1}\right)$, if $A$ or $B$ is a $C$-module, so is $A B$.

\section{$\S 3 . \quad$ Root Vectors}

Definition 3.1. For $n \geq 1$, we set

$$
\begin{aligned}
E_{n \delta+\alpha_{1}} & =\left(T_{1}^{-1} T_{0}^{-1}\right)^{n}\left(e_{1}\right), \\
E_{(2 n+2) \delta-\alpha_{0}} & =\left(T_{1}^{-1} T_{0}^{-1}\right)^{n} T_{1}^{-1}\left(e_{0}\right), \\
E_{2 n \delta+\alpha_{0}} & =\left(T_{0} T_{1}\right)^{n}\left(e_{0}\right), \\
E_{(n+1) \delta-\alpha_{1}} & =\left(T_{0} T_{1}\right)^{n} T_{0}\left(e_{1}\right) .
\end{aligned}
$$

They are homogeneous and the suffices indicate their weights. We call them real root vectors.

Definition 3.2. $\quad$ For $\alpha \in R_{r e}^{+}$such that $(\alpha, \alpha)=\left(\alpha_{i}, \alpha_{i}\right)$ and for $k \geq 0$, we write $E_{\alpha}^{(k)}=E_{\alpha}^{k} /[k]_{i}$ !. They belong to $\mathbf{U}_{\mathbf{Z}}^{+}$by [L, 37.1.3] and [L, 40.2.3]. 
Definition 3.3. For $n \geq 1$, we define the elements $\tilde{\psi}_{n}$ of $\mathbf{U}_{\mathbf{Z}}^{+}$by

$$
\tilde{\psi}_{n}=\left[E_{\delta-\alpha_{1}}, E_{(n-1) \delta+\alpha_{1}}\right]_{q^{-1}} .
$$

We call them imaginary root vectors.

At the end of this section, we shall show that the imaginary root vectors are mutually commutative (Proposition 3.29).

We also set

$$
\tilde{\psi}_{0}=\left(q_{1}-q_{1}^{-1}\right)^{-1}
$$

The following two lemmas follow from Definitions 3.1 and 2.23, and will be often used in this paper.

Lemma 3.4. Under the action of the automorphism $T_{\varpi_{1}}$, we have

$$
\begin{aligned}
T_{\varpi_{1}}\left(E_{n \delta-\alpha_{1}}\right) & =E_{(n+1) \delta-\alpha_{1}} \text { for } n \geq 1, \\
T_{\varpi_{1}}\left(E_{2 n \delta+\alpha_{0}}\right) & =E_{(2 n+2) \delta+\alpha_{0}} \text { for } n \geq 0, \\
T_{\varpi_{1}}\left(E_{n \delta+\alpha_{1}}\right) & =E_{(n-1) \delta+\alpha_{1}} \text { for } n \geq 1, \\
T_{\varpi_{1}}\left(E_{2 n \delta-\alpha_{0}}\right) & =E_{(2 n-2) \delta-\alpha_{0}} \text { for } n \geq 2, \\
T_{\varpi_{1}}^{-1}\left(E_{\delta-\alpha_{1}}\right) & =-k_{1}^{-1} f_{1}, \\
T_{\varpi_{1}}\left(E_{2 \delta-\alpha_{0}}\right) & =-f_{0} k_{0} .
\end{aligned}
$$

Lemma 3.5. Under the action of the anti-involution $T_{1}^{-1} *$, we have

$$
\begin{aligned}
\left(T_{1}^{-1} *\right)\left(E_{n \delta-\alpha_{1}}\right) & =E_{n \delta+\alpha_{1}} \text { for } n \geq 1, \\
\left(T_{1}^{-1} *\right)\left(E_{2 n \delta+\alpha_{0}}\right) & =E_{(2 n+2) \delta-\alpha_{0}} \text { for } n \geq 0, \\
\left(T_{1}^{-1} *\right)\left(E_{\alpha_{1}}\right) & =-k_{1}^{-1} f_{1} .
\end{aligned}
$$

We are going to express the real root vectors without using the braid group action (Corollary 3.10).

Lemma 3.6. We have

(1) $E_{\delta-\alpha_{1}}=\left[E_{\alpha_{0}}, E_{\alpha_{1}}\right]_{q^{-2}}$,

(2) $\tilde{\psi}_{1}=[2]_{1} \sum_{r=0}^{2}(-1)^{r} q_{1}^{-3 r} e_{1}^{(r)} e_{0} e_{1}^{(2-r)}$,

(3) $E_{\delta+\alpha_{1}}=\sum_{r=0}^{3}(-1)^{r} q^{-r} e_{1}^{(r)} e_{0} e_{1}^{(3-r)}$,

(4) $\left[E_{\delta-\alpha_{1}}, f_{1}\right]=[4]_{1} k_{1} E_{\alpha_{0}}$,

(5) $\left[\tilde{\psi}_{1}, f_{1}\right]=[3]_{1} ! k_{1} E_{\delta-\alpha_{1}}$, 
(6) $\left[E_{\delta+\alpha_{1}}, f_{1}\right]=k_{1} \tilde{\psi}_{1}$.

Proof. (1) By Definition 3.1, we have

$$
E_{\delta-\alpha_{1}}=T_{0}\left(e_{1}\right)=\sum_{r=0}^{1}(-1)^{r} q_{0}^{-r} e_{0}^{(1-r)} e_{1} e_{0}^{(r)}=\left[E_{\alpha_{0}}, E_{\alpha_{1}}\right]_{q^{-2}} .
$$

(2) Using Definition 3.3 and (1), we have

$$
\begin{aligned}
\tilde{\psi}_{1} & =\left[E_{\delta-\alpha_{1}}, E_{\alpha_{1}}\right]_{q^{-1}}=\left[\left[E_{\alpha_{0}}, E_{\alpha_{1}}\right]_{q^{-2}}, E_{\alpha_{1}}\right]_{q^{-1}} \\
& =\left(e_{0} e_{1}-q^{-2} e_{1} e_{0}\right) e_{1}-q^{-1} e_{1}\left(e_{0} e_{1}-q^{-2} e_{1} e_{0}\right) \\
& =e_{0} e_{1}^{2}-\left(q^{-1}+q^{-2}\right) e_{1} e_{0} e_{1}+q^{-3} e_{1}^{2} e_{0} \\
& =[2]_{1} \sum_{r=0}^{2}(-1)^{r} q_{1}^{-3 r} e_{1}^{(r)} e_{0} e_{1}^{(2-r)} .
\end{aligned}
$$

(3) By Definition 3.1, we have

$$
\begin{aligned}
E_{\delta+\alpha_{1}} & =T_{1}^{-1} T_{0}^{-1}\left(e_{1}\right)=T_{1}^{-1}\left(\sum_{r=0}^{1}(-1)^{r} q_{0}^{-r} e_{0}^{(r)} e_{1} e_{0}^{(1-r)}\right) \\
& =T_{1}^{-1}\left(\left[e_{1}, e_{0}\right]_{q^{-2}}\right)=\left[-k_{1}^{-1} f_{1}, \sum_{r=0}^{4}(-1)^{r} q_{1}^{-r} e_{1}^{(r)} e_{0} e_{1}^{(4-r)}\right]_{q^{-2}} \\
& =k_{1}^{-1}\left[\sum_{r=0}^{4}(-1)^{r} q_{1}^{-r} e_{1}^{(r)} e_{0} e_{1}^{(4-r)}, f_{1}\right] .
\end{aligned}
$$

Applying Corollary 2.15, we have

$$
\begin{aligned}
E_{\delta+\alpha_{1}}= & k_{1}^{-1} \sum_{r=0}^{4}(-1)^{r} q_{1}^{-r}\left(e_{1}^{(r-1)} \frac{q_{1}^{r-1} k_{1}-q_{1}^{1-r} k_{1}^{-1}}{q_{1}-q_{1}^{-1}} e_{0} e_{1}^{(4-r)}\right. \\
& \left.+e_{1}^{(r)} e_{0} e_{1}^{(3-r)} \frac{q_{1}^{3-r} k_{1}-q_{1}^{r-3} k_{1}^{-1}}{q_{1}-q_{1}^{-1}}\right) \\
= & k_{1}^{-1} \sum_{r=0}^{4}(-1)^{r} q_{1}^{-r} \frac{q_{1}^{1-r} k_{1}-q_{1}^{r-1} k_{1}^{-1}}{q_{1}-q_{1}^{-1}}\left(e_{1}^{(r-1)} e_{0} e_{1}^{(4-r)}+e_{1}^{(r)} e_{0} e_{1}^{(3-r)}\right) \\
= & k_{1}^{-1} \sum_{r=0}^{3}\left((-1)^{r+1} q_{1}^{-r-1} \frac{q_{1}^{-r} k_{1}-q_{1}^{r} k_{1}^{-1}}{q_{1}-q_{1}^{-1}}\right. \\
& \left.+(-1)^{r} q_{1}^{-r} \frac{q_{1}^{1-r} k_{1}-q_{1}^{r-1} k_{1}^{-1}}{q_{1}-q_{1}^{-1}}\right) e_{1}^{(r)} e_{0} e_{1}^{(3-r)}
\end{aligned}
$$


$=\sum_{r=0}^{3}(-1)^{r} q^{-r} e_{1}^{(r)} e_{0} e_{1}^{(3-r)}$.

(4) Using (1), we have

$$
\begin{aligned}
{\left[E_{\delta-\alpha_{1}}, f_{1}\right] } & =\left[\left[e_{0}, e_{1}\right]_{q^{-2}}, f_{1}\right]=\left[e_{0},\left[e_{1}, f_{1}\right]\right]_{q^{-2}}=\left[e_{0}, \frac{k_{1}-k_{1}^{-1}}{q_{1}-q_{1}^{-1}}\right]_{q^{-2}} \\
& =\frac{q^{2} k_{1}-q^{-2} k_{1}^{-1}}{q_{1}-q_{1}^{-1}} e_{0}-q^{-2} \frac{k_{1}-k_{1}^{-1}}{q_{1}-q_{1}^{-1}} e_{0}=[4]_{1} k_{1} E_{\alpha_{0}} .
\end{aligned}
$$

(5) Using Definition 3.3 and (4), we have

$$
\begin{aligned}
{\left[\tilde{\psi}_{1}, f_{1}\right] } & =\left[\left[E_{\delta-\alpha_{1}}, E_{\alpha_{1}}\right]_{q^{-1}}, f_{1}\right] \\
& =\left[\left[E_{\delta-\alpha_{1}}, f_{1}\right], E_{\alpha_{1}}\right]_{q^{-1}}+\left[E_{\delta-\alpha_{1}},\left[E_{\alpha_{1}}, f_{1}\right]\right]_{q^{-1}} \\
& =[4]_{1}\left[k_{1} E_{\alpha_{0}}, E_{\alpha_{1}}\right]_{q^{-1}}+\left[E_{\delta-\alpha_{1}}, \frac{k_{1}-k_{1}^{-1}}{q_{1}-q_{1}^{-1}}\right]_{q^{-1}} \\
& =[4]_{1} k_{1}\left[E_{\alpha_{0}}, E_{\alpha_{1}}\right]_{q^{-2}}+\frac{q k_{1}-q^{-1} k_{1}^{-1}}{q_{1}-q_{1}^{-1}} E_{\delta-\alpha_{1}}-q^{-1} \frac{k_{1}-k_{1}^{-1}}{q_{1}-q_{1}^{-1}} E_{\delta-\alpha_{1}} \\
& =\left([4]_{1}+[2]_{1}\right) k_{1} E_{\delta-\alpha_{1}} \\
& =[3]_{1} ! k_{1} E_{\delta-\alpha_{1}} .
\end{aligned}
$$

(6) Using (3) and Corollary 2.15, we have

$$
\begin{aligned}
{\left[E_{\delta+\alpha_{1}}, f_{1}\right]=} & \sum_{r=0}^{3}(-1)^{r} q^{-r}\left[e_{1}^{(r)} e_{0} e_{1}^{(3-r)}, f_{1}\right] \\
= & \sum_{r=0}^{3}(-1)^{r} q^{-r}\left(e_{1}^{(r-1)} \frac{q_{1}^{r-1} k_{1}-q_{1}^{1-r} k_{1}^{-1}}{q_{1}-q_{1}^{-1}} e_{0} e_{1}^{(3-r)}\right. \\
& \left.+e_{1}^{(r)} e_{0} e_{1}^{(2-r)} \frac{q_{1}^{2-r} k_{1}-q_{1}^{r-2} k_{1}^{-1}}{q_{1}-q_{1}^{-1}}\right) \\
= & \sum_{r=0}^{3}(-1)^{r} q^{-r}\left(\frac{q_{1}^{1-r} k_{1}-q_{1}^{r-1} k_{1}^{-1}}{q_{1}-q_{1}^{-1}} e_{1}^{(r-1)} e_{0} e_{1}^{(3-r)}\right. \\
& +\frac{q_{1}^{2-r} k_{1}-q_{1}^{r-2} k_{1}^{-1}}{\left.q_{1}-q_{1}^{(r)} e_{0} e_{1}^{(2-r)}\right)} \\
= & \sum_{r=0}^{2}\left((-1)^{r+1} q^{-r-1} \frac{q_{1}^{-r} k_{1}-q_{1}^{r} k_{1}^{-1}}{q_{1}-q_{1}^{-1}}\right. \\
& \left.+(-1)^{r} q^{-r} \frac{q_{1}^{2-r} k_{1}-q_{1}^{r-2} k_{1}^{-1}}{q_{1}-q_{1}^{-1}}\right) e_{1}^{(r)} e_{0} e_{1}^{(2-r)}
\end{aligned}
$$




$$
=\sum_{r=0}^{2}(-1)^{r} q_{1}^{-3 r}[2]_{1} k_{1} e_{1}^{(r)} e_{0} e_{1}^{(2-r)},
$$

which is equal to $k_{1} \tilde{\psi}_{1}$ by $(2)$.

Lemma 3.7. Let $n \geq 1$. Then,

$$
\left[E_{n \delta+\alpha_{1}}, f_{1}\right]=k_{1} T_{\varpi_{1}}^{-1}\left(\tilde{\psi}_{n}\right) .
$$

Proof. By Lemma 3.4, we have

$$
\begin{aligned}
{\left[E_{n \delta+\alpha_{1}}, f_{1}\right] } & =\left[E_{n \delta+\alpha_{1}},-k_{1} T_{\varpi_{1}}^{-1}\left(E_{\delta-\alpha_{1}}\right)\right]=k_{1}\left[T_{\varpi_{1}}^{-1}\left(E_{\delta-\alpha_{1}}\right), E_{n \delta+\alpha_{1}}\right]_{q^{-1}} \\
& =k_{1} T_{\varpi_{1}}^{-1}\left(\left[E_{\delta-\alpha_{1}}, E_{(n-1) \delta+\alpha_{1}}\right]_{q^{-1}}\right) .
\end{aligned}
$$

Hence, the lemma follows from Definition 3.3.

Lemma 3.8. We have

(1) $T_{\varpi_{1}}\left(\tilde{\psi}_{1}\right)=\tilde{\psi}_{1}$,

(2) $\left(T_{1}^{-1} *\right)\left(\tilde{\psi}_{1}\right)=\tilde{\psi}_{1}$.

Proof. By Lemma 3.6 (6) and Lemma 3.7, we have $T_{\varpi_{1}}^{-1}\left(\tilde{\psi}_{1}\right)=\tilde{\psi}_{1}$, obtaining (1). By Definition 3.3, we have

$$
\begin{aligned}
\left(T_{1}^{-1} *\right)\left(\tilde{\psi}_{1}\right) & =\left(T_{1}^{-1} *\right)\left(\left[E_{\delta-\alpha_{1}}, E_{\alpha_{1}}\right]_{q^{-1}}\right)=\left[-k_{1}^{-1} f_{1}, E_{\delta+\alpha_{1}}\right]_{q^{-1}} \\
& =k_{1}^{-1}\left[E_{\delta+\alpha_{1}}, f_{1}\right] .
\end{aligned}
$$

Applying Lemma 3.6 (6), we obtain (2).

Lemma 3.9. We have

(1) $\left[E_{2 \delta-\alpha_{0}}, E_{\alpha_{1}}\right]_{q^{2}}=0$,

(2) $\left[E_{\alpha_{0}}, E_{\delta-\alpha_{1}}\right]_{q^{2}}=0$,

(3) $\left[\tilde{\psi}_{1}, E_{\alpha_{1}}\right]=[3]_{1} ! E_{\delta+\alpha_{1}}$,

(4) $\left[E_{\alpha_{0}}, \tilde{\psi}_{1}\right]=(q-1)[3]_{1} E_{\delta-\alpha_{1}}^{2}$,

(5) $\left[E_{\delta+\alpha_{1}}, E_{\alpha_{1}}\right]_{q}=[4]_{1} E_{2 \delta-\alpha_{0}}$. 
Proof. (1) By Definition 3.1, we have

$$
\begin{aligned}
{\left[E_{2 \delta-\alpha_{0}}, E_{\alpha_{1}}\right]_{q^{2}} } & =\left[T_{1}^{-1}\left(E_{\alpha_{0}}\right), E_{\alpha_{1}}\right]_{q^{2}}=T_{1}^{-1}\left(\left[E_{\alpha_{0}}, T_{1} E_{\alpha_{1}}\right]_{q^{2}}\right) \\
& =T_{1}^{-1}\left(\left[e_{0},-f_{1} k_{1}\right]_{q^{2}}\right)=-T_{1}^{-1}\left(\left[e_{0}, f_{1}\right] k_{1}\right)=0 .
\end{aligned}
$$

(2) By Definition 3.1, we have

$$
\begin{aligned}
{\left[E_{\alpha_{0}}, E_{\delta-\alpha_{1}}\right]_{q^{2}} } & =\left[E_{\alpha_{0}}, T_{0}\left(E_{\alpha_{1}}\right)\right]_{q^{2}}=T_{0}\left(\left[T_{0}^{-1}\left(E_{\alpha_{0}}\right), E_{\alpha_{1}}\right]_{q^{2}}\right) \\
& =T_{0}\left(\left[-k_{0}^{-1} f_{0}, e_{1}\right]_{q^{2}}\right)=T_{0}\left(k_{0}^{-1}\left[e_{1}, f_{0}\right]\right)=0 .
\end{aligned}
$$

(3) Using Lemmas 3.8 (2) and 3.6 (5), we have

$$
\left(T_{1}^{-1} *\right)\left(\left[\tilde{\psi}_{1}, E_{\alpha_{1}}\right]\right)=\left[-k_{1}^{-1} f_{1}, \tilde{\psi}_{1}\right]=k_{1}^{-1}\left[\tilde{\psi}_{1}, f_{1}\right]=[3]_{1} ! E_{\delta-\alpha_{1}} .
$$

Applying Lemma 3.5, we obtain (3).

(4) Using Definition 3.3 and Lemma 2.11, we have

$$
\begin{aligned}
{\left[E_{\alpha_{0}}, \tilde{\psi}_{1}\right]=} & {\left[E_{\alpha_{0}}, E_{\delta-\alpha_{1}} E_{\alpha_{1}}-q^{-1} E_{\alpha_{1}} E_{\delta-\alpha_{1}}\right] } \\
= & {\left[E_{\alpha_{0}}, E_{\delta-\alpha_{1}}\right]_{q^{2}} E_{\alpha_{1}}+E_{\delta-\alpha_{1}}\left[E_{\alpha_{0}}, E_{\alpha_{1}}\right]_{q^{-2}} q^{2} } \\
& -\left[E_{\alpha_{0}}, E_{\alpha_{1}}\right]_{q^{-2}} E_{\delta-\alpha_{1}} q^{-1}-E_{\alpha_{1}}\left[E_{\alpha_{0}}, E_{\delta-\alpha_{1}}\right]_{q^{2}} q^{-3} .
\end{aligned}
$$

The first term and the last one vanish by (2). Thus, by Lemma 3.6 (1), we obtain (4).

(5) By Lemma 3.6 (4), we have

$$
\left(T_{1}^{-1} *\right)\left(\left[E_{\delta+\alpha_{1}}, E_{\alpha_{1}}\right]_{q}\right)=\left[-k_{1}^{-1} f_{1}, E_{\delta-\alpha_{1}}\right]_{q}=k_{1}^{-1}\left[E_{\delta-\alpha_{1}}, f_{1}\right]=[4]_{1} E_{\alpha_{0}} .
$$

Applying Lemma 3.5, we obtain (5).

Corollary 3.10. We have

(1) $\left[E_{(2 n+2) \delta-\alpha_{0}}, E_{n \delta+\alpha_{1}}\right]_{q^{2}}=0$ for $n \geq 0$,

(2) $\left[E_{n \delta-\alpha_{1}}, E_{2 n \delta+\alpha_{0}}\right]_{q^{2}}=0$ for $n \geq 1$,

(3) $\left[E_{2 n \delta+\alpha_{0}}, E_{(n+1) \delta-\alpha_{1}}\right]_{q^{2}}=0$ for $n \geq 0$,

(4) $\left[E_{n \delta+\alpha_{1}}, E_{2 n \delta-\alpha_{0}}\right]_{q^{2}}=0$ for $n \geq 1$,

(5) $\left[E_{(n+1) \delta+\alpha_{1}}, E_{n \delta+\alpha_{1}}\right]_{q}=[4]_{1} E_{(2 n+2) \delta-\alpha_{0}}$ for $n \geq 0$,

(6) $\left[E_{n \delta-\alpha_{1}}, E_{(n+1) \delta-\alpha_{1}}\right]_{q}=[4]_{1} E_{2 n \delta+\alpha_{0}}$ for $n \geq 1$,

(7) $\left[\tilde{\psi}_{1}, E_{n \delta+\alpha_{1}}\right]=[3]_{1} ! E_{(n+1) \delta+\alpha_{1}}$ for $n \geq 0$, 
(8) $\left[E_{n \delta-\alpha_{1}}, \tilde{\psi}_{1}\right]=[3]_{1} ! E_{(n+1) \delta-\alpha_{1}}$ for $n \geq 1$,

(9) $\left[E_{2 n \delta+\alpha_{0}}, \tilde{\psi}_{1}\right]=(q-1)[3]_{1} E_{(n+1) \delta-\alpha_{1}}^{2}$ for $n \geq 0$,

(10) $\left[\tilde{\psi}_{1}, E_{2 n \delta-\alpha_{0}}\right]=(q-1)[3]_{1} E_{n \delta+\alpha_{1}}^{2} \quad$ for $n \geq 1$.

Proof. We use Lemmas 3.4, 3.5 and 3.8.

Applying $T_{\varpi_{1}}^{n}$ to Lemma 3.9 (1), we obtain (1).

Applying $T_{1}^{-1} *$ to (1) with $n \geq 1$, we obtain (2).

Applying $T_{\varpi_{1}}^{n}$ to Lemma 3.9 (2), we obtain (3).

Applying $T_{1}^{-1} *$ to (3) with $n$ replaced by $n-1$, we obtain (4).

Applying $T_{\varpi_{1}}^{n}$ to Lemma 3.9 (5), we obtain (5).

Applying $T_{1}^{-1} *$ to $(5)$, we obtain (6).

Applying $T_{\varpi_{1}}^{n}$ to Lemma 3.9 (3), we obtain (7).

Applying $T_{1}^{-1} *$ to $(7)$, we obtain (8).

Applying $T_{\varpi_{1}}^{n}$ to Lemma 3.9 (4), we obtain (9).

Applying $T_{1}^{-1} *$ to (9) with $n$ replaced by $n-1$, we obtain (10).

We introduce certain elements of $\mathbf{Z}\left[q, q^{-1}\right]$ that will be used for the commutation relations between the root vectors.

Definition 3.11. For $n \in \mathbf{Z}$, we define the elements $b_{n}$ of $\mathbf{Z}\left[q, q^{-1}\right]$ as follows: for $i \in \mathbf{Z}$, we set

$$
\begin{aligned}
b_{2 i} & =\left(1-q+q^{2}\right)^{-1}(1-q)\left((-q)^{-i}-q^{2 i}\left(q+q^{-1}\right)\right), \\
b_{2 i+1} & =\left(1-q+q^{2}\right)^{-1}\left((-q)^{-i}+q^{2 i+1}(q-1)\right) .
\end{aligned}
$$

Note that the roots $e^{ \pm \pi i / 3}$ of $1-q+q^{2}$ are roots of both of the last factors.

Remark 3.12. It follows from Definition 3.11 that $b_{-n}=-q^{-1} \overline{b_{n}}$ for $n \in \mathbf{Z}$ where - is the automorphism of $\mathbf{Z}\left[q, q^{-1}\right]$ given by $\bar{q}=q^{-1}$.

Example 3.13. We have

$$
\begin{aligned}
& b_{-2}=1-q^{-3}, \quad b_{-1}=-q^{-1}, \quad b_{0}=1-q^{-1} \\
& b_{1}=1, \quad b_{2}=q^{2}-q^{-1}=(q-1)[3]_{1} \\
& b_{3}=q^{2}-1-q^{-1}=b_{2}-1, \quad b_{4}=q^{4}-q-1+q^{-2} .
\end{aligned}
$$

Lemma 3.14. Let $i \in \mathbf{Z}$. Then,

(1) $b_{2 i+1}+b_{2 i-1}=b_{2 i}$, 
(2) $q b_{2 i}+\left(q+q^{-1}\right)(q-1) b_{2 i+1}=b_{2(i+1)}$,

(3) $q b_{2 i}+b_{2} b_{2(i+1)}=b_{2(i+2)}$.

Proof. We can directly check this by using Definition 3.11 .

Lemma 3.15. Let $k, l \in \mathbf{Z}$. Then,

(1) $b_{2(k+1)} b_{2(l+1)}+q b_{2 k} b_{2 l}=b_{2(k+l+2)}-b_{2(k+l)}$,

(2) $b_{2 k} b_{2 l+1}+q b_{2(k-1)} b_{2(l-1)+1}=b_{2(k+l)}-b_{2(k+l-1)}$,

(3) $b_{2 k} b_{2 l}-\left(q+q^{-1}\right)(q-1) b_{2 k-1} b_{2 l-1}=b_{2(k+l)}-b_{2(k+l-1)}$.

Proof. We can directly check (1) and (2) by using Definition 3.11. We check (3): by Lemma $3.14((2),(1))$, the left hand side is equal to

$$
\begin{aligned}
b_{2 k} b_{2 l}-\left(b_{2 k}-q b_{2 k-2}\right) b_{2 l-1} & =b_{2 k}\left(b_{2 l}-b_{2 l-1}\right)+q b_{2(k-1)} b_{2 l-1} \\
& =b_{2 k} b_{2 l+1}+q b_{2(k-1)} b_{2 l-1}
\end{aligned}
$$

which is equal to the right hand side by (2).

Lemma 3.16. Let $s \in \mathbf{Z}, l \in \mathbf{Z}_{\geq 1}$. Then,

$$
\begin{aligned}
q b_{2 s} & +(1+q) \sum_{i=1}^{l-1} b_{2 i} b_{2(i+s)} \theta(l \geq 2)+b_{2 l} b_{2(l+s)}=b_{2(2 l+s)} \\
q b_{2 s} & +(1+q) \sum_{i=1}^{l-1} b_{2 i} b_{2(i+s)} \theta(l \geq 2) \\
& +\left(q+q^{-1}\right)(q-1) b_{2 l-1} b_{2(l+s)-1}=b_{2(2 l+s-1)} .
\end{aligned}
$$

Proof. (1) is checked by the induction on $l$ : the case where $l=1$ is nothing but Lemma 3.14 (3), and the induction proceeds by Lemma 3.15 (1). (2) follows from (1) and Lemma 3.15 (3).

Lemma 3.17. Let $l \geq 1$. Then,

$$
\begin{aligned}
& \sum_{i=1}^{l} b_{2 i}[2 l-2 i+1]_{1} q^{i}=(q-1) q^{l}[l][2 l+1]_{1}, \\
& \sum_{i=1}^{l} b_{2 i-1}[2 l-2 i+1]_{1} q^{i}=q^{l}[l]_{0} .
\end{aligned}
$$


Proof. The lemma is reduced to the following identities in $\mathbf{Z}\left[q, q^{-1}\right][[u]]$ :

$$
\begin{aligned}
\left(\sum_{i \geq 1} b_{2 i} q^{i} u^{i}\right)\left(\sum_{k \geq 0}[2 k+1]_{1} u^{k}\right) & =(q-1) \sum_{l \geq 1} q^{l}[l][2 l+1]_{1} u^{l}, \\
\left(\sum_{i \geq 1} b_{2 i-1} q^{i} u^{i}\right)\left(\sum_{k \geq 0}[2 k+1]_{1} u^{k}\right) & =\sum_{l \geq 1} q^{l}[l]_{0} u^{l} .
\end{aligned}
$$

Using Definition 3.11, we can directly check

$$
\begin{aligned}
\sum_{i \geq 1} b_{2 i} q^{i} u^{i} & =\frac{(q-1) u\left(q^{2} u+\left(q^{2}+q+1\right)\right)}{(1+u)\left(1-q^{3} u\right)}, \\
\sum_{i \geq 1} b_{2 i-1} q^{i} u^{i} & =\frac{q u(1-q u)}{(1+u)\left(1-q^{3} u\right)} .
\end{aligned}
$$

We can also directly check

$$
\begin{aligned}
\sum_{k \geq 0}[2 k+1]_{1} u^{k} & =\frac{1+u}{(1-q u)\left(1-q^{-1} u\right)}, \\
\sum_{l \geq 1} q^{l}[l][2 l+1]_{1} u^{l} & =\frac{u\left(q^{2} u+\left(q^{2}+q+1\right)\right)}{\left(1-q^{3} u\right)(1-q u)\left(1-q^{-1} u\right)}, \\
\sum_{l \geq 1} q^{l}[l]_{0} u^{l} & =\frac{q u}{\left(1-q^{3} u\right)\left(1-q^{-1} u\right)} .
\end{aligned}
$$

Hence, the lemma follows.

We shall study the commutation relations between the real root vectors of height 1 (Corollary $3.20(1)$ ) and of height -1 (Corollary $3.20(2)$ ).

Lemma 3.18. Let $n \geq 2$. Then,

$$
\begin{aligned}
{\left[E_{n \delta+\alpha_{1}}, E_{\alpha_{1}}\right]_{q}=} & {\left[\tilde{\psi}_{1},\left[E_{(n-1) \delta+\alpha_{1}}, E_{\alpha_{1}}\right]_{q}\right]\left([3]_{1} !\right)^{-1} } \\
& -T_{\varpi_{1}}^{-1}\left(\left[E_{(n-2) \delta+\alpha_{1}}, E_{\alpha_{1}}\right]_{q}\right) .
\end{aligned}
$$

Proof. Using Corollary 3.10 (7) and Lemma 2.11, we have

$$
\begin{aligned}
{\left[E_{n \delta+\alpha_{1}}, E_{\alpha_{1}}\right]_{q}[3]_{1} !=} & {\left[\tilde{\psi}_{1} E_{(n-1) \delta+\alpha_{1}}-E_{(n-1) \delta+\alpha_{1}} \tilde{\psi}_{1}, E_{\alpha_{1}}\right]_{q} } \\
= & \tilde{\psi}_{1}\left[E_{(n-1) \delta+\alpha_{1}}, E_{\alpha_{1}}\right]_{q}+\left[\tilde{\psi}_{1}, E_{\alpha_{1}}\right] E_{(n-1) \delta+\alpha_{1}} q \\
& -E_{(n-1) \delta+\alpha_{1}}\left[\tilde{\psi}_{1}, E_{\alpha_{1}}\right]-\left[E_{(n-1) \delta+\alpha_{1}}, E_{\alpha_{1}}\right] \tilde{\psi}_{1} \\
= & {\left[\tilde{\psi}_{1},\left[E_{(n-1) \delta+\alpha_{1}}, E_{\alpha_{1}}\right]_{q}\right]-\left[E_{(n-1) \delta+\alpha_{1}}, E_{\delta+\alpha_{1}}\right]_{q}[3]_{1} ! }
\end{aligned}
$$

Applying Lemma 3.4, we obtain the lemma. 
Proposition 3.19. Let $n \geq 1$. Then,

$$
\begin{aligned}
{\left[E_{n \delta+\alpha_{1}}, E_{\alpha_{1}}\right]_{q}=} & (1+q) \sum_{i=1}^{[(n-1) / 2]} b_{2 i} E_{i \delta+\alpha_{1}} E_{(n-i) \delta+\alpha_{1}} \theta(n \geq 3) \\
& +\left\{\begin{array}{l}
b_{n} E_{\frac{n}{2}}^{2} \delta+\alpha_{1} \\
{[4]_{1} b_{n} E_{(n+1) \delta-\alpha_{0}} \text { if } n \text { is even } n \text { is odd. }}
\end{array}\right.
\end{aligned}
$$

Proof. We argue by the induction on $n$. The case where $n=1$ is Lemma 3.9 (5). The case where $n=2$ follows from Lemma 3.18 with $n=2$ and Lemma $3.9(5)$ :

$$
\begin{aligned}
{\left[E_{2 \delta+\alpha_{1}}, E_{\alpha_{1}}\right]_{q} } & =\left[\tilde{\psi}_{1},\left[E_{\delta+\alpha_{1}}, E_{\alpha_{1}}\right]_{q}\right]\left([3]_{1} !\right)^{-1}-(1-q) T_{\varpi_{1}}^{-1}\left(E_{\alpha_{1}}^{2}\right) \\
& =\left[\tilde{\psi}_{1},[4]_{1} E_{2 \delta-\alpha_{0}}\right]\left([3]_{1} !\right)^{-1}+(q-1) E_{\delta+\alpha_{1}}^{2} \\
& =(q-1)\left([4]_{1}[2]_{1}^{-1}+1\right) E_{\delta+\alpha_{1}}^{2}=b_{2} E_{\delta+\alpha_{1}}^{2} .
\end{aligned}
$$

Now, assume that $n \geq 3$. First, we consider the case where $n=2 m+1$ with $m \geq 1$. Using Lemma 3.18, the induction hypothesis, and Corollary 3.10 (7), we have

$$
\begin{aligned}
{\left[E_{(2 m+1) \delta+\alpha_{1}}, E_{\alpha_{1}}\right]_{q} } & \\
= & {\left[\tilde{\psi}_{1},\left[E_{2 m \delta+\alpha_{1}}, E_{\alpha_{1}}\right]_{q}\right]\left([3]_{1} !\right)^{-1}-T_{\varpi_{1}}^{-1}\left(\left[E_{(2 m-1) \delta+\alpha_{1}}, E_{\alpha_{1}}\right]_{q}\right) } \\
= & {\left[\tilde{\psi}_{1},(1+q) \sum_{i=1}^{m-1} b_{2 i} E_{i \delta+\alpha_{1}} E_{(2 m-i) \delta+\alpha_{1}}+b_{2 m} E_{m \delta+\alpha_{1}}^{2}\right]\left([3]_{1} !\right)^{-1} } \\
& -T_{\varpi_{1}}^{-1}\left((1+q) \sum_{i=1}^{m-1} b_{2 i} E_{i \delta+\alpha_{1}} E_{(2 m-i-1) \delta+\alpha_{1}}+[4]_{1} b_{2 m-1} E_{2 m \delta-\alpha_{0}}\right) \\
= & (1+q) \sum_{i=1}^{m-1} b_{2 i}\left(E_{(i+1) \delta+\alpha_{1}} E_{(2 m-i) \delta+\alpha_{1}}+E_{i \delta+\alpha_{1}} E_{(2 m-i+1) \delta+\alpha_{1}}\right) \\
& +b_{2 m}\left(E_{(m+1) \delta+\alpha_{1}} E_{m \delta+\alpha_{1}}+E_{m \delta+\alpha_{1}} E_{(m+1) \delta+\alpha_{1}}\right) \\
& -(1+q) \sum_{i=1}^{m-1} b_{2 i} E_{(i+1) \delta+\alpha_{1}} E_{(2 m-i) \delta+\alpha_{1}}-[4]_{1} b_{2 m-1} E_{(2 m+2) \delta-\alpha_{0}} \\
= & (1+q) \sum_{i=1}^{m-1} b_{2 i} E_{i \delta+\alpha_{1}} E_{(2 m-i+1) \delta+\alpha_{1}} \\
& +b_{2 m}\left((1+q) E_{m \delta+\alpha_{1}} E_{(m+1) \delta+\alpha_{1}}+[4]_{1} E_{(2 m+2) \delta-\alpha_{0}}\right) \\
& -[4]_{1} b_{2 m-1} E_{(2 m+2) \delta-\alpha_{0}} .
\end{aligned}
$$


By Corollary 3.10 (5), this is equal to

$$
(1+q) \sum_{i=1}^{m} b_{2 i} E_{i \delta+\alpha_{1}} E_{(2 m+1-i) \delta+\alpha_{1}}+[4]_{1}\left(b_{2 m}-b_{2 m-1}\right) E_{(2 m+2) \delta-\alpha_{0}} .
$$

It remains to apply Lemma 3.14 (1). Next, we consider the case where $n=2 m$ with $m \geq 2$. Using Lemma 3.18, the induction hypothesis, and Corollary 3.10 $((7),(10))$, we have

$$
\begin{aligned}
{[} & \left.E_{2 m \delta+\alpha_{1}}, E_{\alpha_{1}}\right]_{q} \\
= & {\left[\tilde{\psi}_{1},\left[E_{(2 m-1) \delta+\alpha_{1}}, E_{\alpha_{1}}\right]_{q}\right]\left([3]_{1} !\right)^{-1}-T_{\varpi_{1}}^{-1}\left(\left[E_{(2 m-2) \delta+\alpha_{1}}, E_{\alpha_{1}}\right]_{q}\right) } \\
= & {\left[\tilde{\psi}_{1},(1+q) \sum_{i=1}^{m-1} b_{2 i} E_{i \delta+\alpha_{1}} E_{(2 m-1-i) \delta+\alpha_{1}}+[4]_{1} b_{2 m-1} E_{2 m \delta-\alpha_{0}}\right]\left([3]_{1} !\right)^{-1} } \\
& -T_{\varpi_{1}}^{-1}\left((1+q) \sum_{i=1}^{m-2} b_{2 i} E_{i \delta+\alpha_{1}} E_{(2 m-2-i) \delta+\alpha_{1}}+b_{2 m-2} E_{(m-1) \delta+\alpha_{1}}^{2}\right) \\
= & (1+q) \sum_{i=1}^{m-1} b_{2 i}\left(E_{(i+1) \delta+\alpha_{1}} E_{(2 m-1-i) \delta+\alpha_{1}}+E_{i \delta+\alpha_{1}} E_{(2 m-i) \delta+\alpha_{1}}\right) \\
& +\left(q+q q^{-1}\right)(q-1) b_{2 m-1} E_{m \delta+\alpha_{1}}^{2} \\
& -(1+q) \sum_{i=1}^{m-2} b_{2 i} E_{(i+1) \delta+\alpha_{1}} E_{(2 m-1-i) \delta+\alpha_{1}}-b_{2 m-2} E_{m \delta+\alpha_{1}}^{2} \\
= & (1+q) \sum_{i=1}^{m-1} b_{2 i} E_{i \delta+\alpha_{1}} E_{(2 m-i) \delta+\alpha_{1}} \\
& +\left(q b_{2 m-2}+\left(q+q^{-1}\right)(q-1) b_{2 m-1}\right) E_{m \delta+\alpha_{1}}^{2} .
\end{aligned}
$$

It remains to apply Lemma 3.14 (2). The proposition is proved.

\section{Corollary 3.20.}

(1) Let $k>l \geq 0$. Then,

$$
\begin{aligned}
{\left[E_{k \delta+\alpha_{1}}, E_{l \delta+\alpha_{1}}\right]_{q}=} & (1+q) \sum_{i=1}^{[(k-l-1) / 2]} b_{2 i} E_{(l+i) \delta+\alpha_{1}} E_{(k-i) \delta+\alpha_{1}} \theta(k-l \geq 3) \\
& +\left\{\begin{array}{l}
b_{k-l} E_{\frac{k+l}{2} \delta+\alpha_{1}}^{2} \text { if } k-l \text { is even, } \\
{[4]_{1} b_{k-l} E_{(k+l+1) \delta-\alpha_{0}} \text { if } k-l \text { is odd. }}
\end{array}\right.
\end{aligned}
$$


(2) Let $l>k \geq 1$. Then,

$$
\begin{aligned}
{\left[E_{k \delta-\alpha_{1}}, E_{l \delta-\alpha_{1}}\right]_{q}=} & (1+q) \sum_{i=1}^{[(l-k-1) / 2]} b_{2 i} E_{(l-i) \delta-\alpha_{1}} E_{(k+i) \delta-\alpha_{1}} \theta(l-k \geq 3) \\
& +\left\{\begin{array}{l}
b_{l-k} E_{\frac{k+l}{2} \delta-\alpha_{1}}^{2} \text { if } l-k \text { is even, } \\
{[4]_{1} b_{l-k} E_{(k+l-1) \delta+\alpha_{0}} \text { if } l-k \text { is odd } .}
\end{array}\right.
\end{aligned}
$$

Proof. Applying $T_{\varpi 1}^{-(k-l)}$ to Proposition 3.19, we obtain (1). Applying $T_{1}^{-1} *$ to (1) with $k$ and $l$ exchanged, we obtain (2).

We shall prove that the imaginary root vectors are invariant under $T_{\varpi_{1}}$ and $T_{1}^{-1} *$ (Proposition $3.26((1),(3))$ ) and are mutually commutative (Proposition 3.29). As by-products, we shall obtain the commutation relations between the imaginary root vectors and the real root vectors of height 1 or -1 (Proposition $3.26((4),(5)))$, and the ones between the real root vectors of height 1 and -1 (Proposition $3.26(2)$ ), of height 1 and -2 (Corollary $3.27(1)$ ), and of height 2 and -1 (Corollary $3.27(2)$ ); as for the ones between the real root vectors of height 2 and -2 , see Appendix A. First, we prove several lemmas.

Lemma 3.21. Let $n \geq 1$. Then,

$$
\begin{aligned}
{\left[E_{\alpha_{0}}, E_{n \delta+\alpha_{1}}\right]_{q^{-2}}[3]_{1} !=} & \left(q_{1}-q_{1}^{-1}\right)[3]_{1} ! \tilde{\psi}_{n} E_{\delta-\alpha_{1}}+(q-1)[3]_{1}\left[E_{\delta-\alpha_{1}}, \tilde{\psi}_{n}\right] \\
& -\left[\left[E_{\alpha_{0}}, E_{(n-1) \delta+\alpha_{1}}\right]_{q^{-2}}, \tilde{\psi}_{1}\right] .
\end{aligned}
$$

Proof. Using Corollary 3.10 (7), Lemmas 2.11 and 3.9 (4), we have

$$
\begin{aligned}
{\left[E_{\alpha_{0}}, E_{n \delta+\alpha_{1}}\right]_{q^{-2}}[3]_{1} ! } \\
=\left[E_{\alpha_{0}}, \tilde{\psi}_{1} E_{(n-1) \delta+\alpha_{1}}-E_{(n-1) \delta+\alpha_{1}} \tilde{\psi}_{1}\right]_{q^{-2}} \\
=\left[E_{\alpha_{0}}, \tilde{\psi}_{1}\right] E_{(n-1) \delta+\alpha_{1}}+\tilde{\psi}_{1}\left[E_{\alpha_{0}}, E_{(n-1) \delta+\alpha_{1}}\right]_{q^{-2}} \\
\\
\quad-\left[E_{\alpha_{0}}, E_{(n-1) \delta+\alpha_{1}}\right]_{q^{-2}} \tilde{\psi}_{1}-E_{(n-1) \delta+\alpha_{1}}\left[E_{\alpha_{0}}, \tilde{\psi}_{1}\right] q^{-2} \\
=\left[\tilde{\psi}_{1},\left[E_{\alpha_{0}}, E_{(n-1) \delta+\alpha_{1}}\right]_{q^{-2}}\right]+(q-1)[3]_{1}\left[E_{\delta-\alpha_{1}}^{2}, E_{(n-1) \delta+\alpha_{1}}\right]_{q^{-2}} .
\end{aligned}
$$

We rewrite the last term: using Definition 3.3 and Lemma 2.11, we have

$$
\begin{aligned}
& {\left[E_{\delta-\alpha_{1}}^{2}, E_{(n-1) \delta+\alpha_{1}}\right]_{q^{-2}}} \\
& \quad=E_{\delta-\alpha_{1}}\left[E_{\delta-\alpha_{1}}, E_{(n-1) \delta+\alpha_{1}}\right]_{q^{-1}}+\left[E_{\delta-\alpha_{1}}, E_{(n-1) \delta+\alpha_{1}}\right]_{q^{-1}} E_{\delta-\alpha_{1}} q^{-1} \\
& \quad=E_{\delta-\alpha_{1}} \tilde{\psi}_{n}+\tilde{\psi}_{n} E_{\delta-\alpha_{1}} q^{-1} \\
& \quad=\left(1+q^{-1}\right) \tilde{\psi}_{n} E_{\delta-\alpha_{1}}+\left[E_{\delta-\alpha_{1}}, \tilde{\psi}_{n}\right] .
\end{aligned}
$$

Hence, we obtain the lemma. 
Lemma 3.22. Let $n \geq 2$. Then,

$$
T_{\varpi_{1}}\left(\tilde{\psi}_{n}\right)=\tilde{\psi}_{n}+\left[\tilde{\psi}_{n-1}, \tilde{\psi}_{1}\right]\left([3]_{1} !\right)^{-1} .
$$

Proof. Using Definition 3.3, Corollary 3.10 ((7), (8)) and Lemma 2.11, we have

$$
\begin{aligned}
\tilde{\psi}_{n}[3]_{1} != & {\left[E_{\delta-\alpha_{1}}, E_{(n-1) \delta+\alpha_{1}}\right]_{q^{-1}}[3]_{1} ! } \\
= & {\left[E_{\delta-\alpha_{1}}, \tilde{\psi}_{1} E_{(n-2) \delta+\alpha_{1}}-q^{-1} E_{(n-2) \delta+\alpha_{1}} \tilde{\psi}_{1}\right]_{q^{-1}} } \\
= & {\left[E_{\delta-\alpha_{1}}, \tilde{\psi}_{1}\right] E_{(n-2) \delta+\alpha_{1}}+\tilde{\psi}_{1}\left[E_{\delta-\alpha_{1}}, E_{(n-2) \delta+\alpha_{1}}\right]_{q^{-1}} } \\
& -\left[E_{\delta-\alpha_{1}}, E_{(n-2) \delta+\alpha_{1}}\right]_{q^{-1}} \tilde{\psi}_{1}-E_{(n-2) \delta+\alpha_{1}}\left[E_{\delta-\alpha_{1}}, \tilde{\psi}_{1}\right] q^{-1} \\
= & {\left[\tilde{\psi}_{1},\left[E_{\delta-\alpha_{1}}, E_{(n-2) \delta+\alpha_{1}}\right]_{q^{-1}}\right]+\left[E_{2 \delta-\alpha_{1}}, E_{(n-2) \delta+\alpha_{1}}\right]_{q^{-1}}[3]_{1} ! } \\
= & {\left[\tilde{\psi}_{1}, \tilde{\psi}_{n-1}\right]+T_{\varpi_{1}}\left(\tilde{\psi}_{n}\right)[3]_{1} ! }
\end{aligned}
$$

Hence, we obtain the lemma.

Lemma 3.23. Let $n \geq 1$. Then,

$$
\begin{aligned}
{\left[\tilde{\psi}_{n}, E_{\alpha_{1}}\right]=} & \left(1+q^{-1}\right) q b_{2} E_{n \delta+\alpha_{1}} \tilde{\psi}_{0}+\left(1+q^{-1}\right) q b_{0} E_{(n-1) \delta+\alpha_{1}} \tilde{\psi}_{1} \\
& +\left[E_{\delta-\alpha_{1}},\left[E_{(n-1) \delta+\alpha_{1}}, E_{\alpha_{1}}\right]_{q}\right]_{q^{-2}} .
\end{aligned}
$$

Proof. Using Definition 3.3, Corollary 3.10 (7) and Lemma 2.11, we see that the left hand side is equal to

$$
\begin{aligned}
& {\left[E_{\delta-\alpha_{1}} E_{(n-1) \delta+\alpha_{1}}-q^{-1} E_{(n-1) \delta+\alpha_{1}} E_{\delta-\alpha_{1}}, E_{\alpha_{1}}\right]} \\
& =E_{\delta-\alpha_{1}}\left[E_{(n-1) \delta+\alpha_{1}}, E_{\alpha_{1}}\right]_{q}+\left[E_{\delta-\alpha_{1}}, E_{\alpha_{1}}\right]_{q^{-1}} E_{(n-1) \delta+\alpha_{1}} q \\
& \quad-E_{(n-1) \delta+\alpha_{1}}\left[E_{\delta-\alpha_{1}}, E_{\alpha_{1}}\right]_{q^{-1}} q^{-1}-\left[E_{(n-1) \delta+\alpha_{1}}, E_{\alpha_{1}}\right]_{q} E_{\delta-\alpha_{1}} q^{-2} \\
& =\left[E_{\delta-\alpha_{1}},\left[E_{(n-1) \delta+\alpha_{1}}, E_{\alpha_{1}}\right]_{q}\right]_{q^{-2}}+\left(q-q^{-1}\right) E_{(n-1) \delta+\alpha_{1}} \tilde{\psi}_{1}+q[3]_{1} ! E_{n \delta+\alpha_{1}} .
\end{aligned}
$$

Hence, we obtain the lemma.

Lemma 3.24. Let $k, l \geq 0$. Then,

$$
\left[E_{\delta-\alpha_{1}}, E_{k \delta+\alpha_{1}} E_{l \delta+\alpha_{1}}\right]_{q^{-2}}=q^{-1} E_{k \delta+\alpha_{1}} \tilde{\psi}_{l+1}+E_{l \delta+\alpha_{1}} \tilde{\psi}_{k+1}+\left[\tilde{\psi}_{k+1}, E_{l \delta+\alpha_{1}}\right] \text {. }
$$

Proof. Using Lemma 2.11 and Definition 3.3, we see that the left hand side is equal to

$$
\begin{gathered}
{\left[E_{\delta-\alpha_{1}}, E_{k \delta+\alpha_{1}}\right]_{q^{-1}} E_{l \delta+\alpha_{1}}+E_{k \delta+\alpha_{1}}\left[E_{\delta-\alpha_{1}}, E_{l \delta+\alpha_{1}}\right]_{q^{-1}} q^{-1}} \\
=q^{-1} E_{k \delta+\alpha_{1}} \tilde{\psi}_{l+1}+\tilde{\psi}_{k+1} E_{l \delta+\alpha_{1}} .
\end{gathered}
$$

Hence, we obtain the lemma. 
Lemma 3.25. We have

(1) $r_{0}\left(E_{\alpha}\right)=0$ for $\alpha \in R_{r e}^{+} \backslash\left\{\alpha_{0}\right\}$,

(2) $r_{0}\left(\tilde{\psi}_{n}\right)=0$ for $n \geq 1$.

Proof. Using Lemma $3.6(1)$, we have $r_{0}\left(E_{\delta-\alpha_{1}}\right)=0$. Hence, by Definition 3.3, we have $r_{0}\left(\tilde{\psi}_{1}\right)=0$. Applying Corollary 3.10, we obtain (1). By (1) and Definition 3.3, we obtain (2).

Proposition 3.26. Let $n \geq 1$. Then,

(1) $T_{\varpi_{1}}\left(\tilde{\psi}_{n}\right)=\tilde{\psi}_{n}$,

(2) $\left[E_{k \delta-\alpha_{1}}, E_{l \delta+\alpha_{1}}\right]_{q^{-1}}=\tilde{\psi}_{n}$ for $k \geq 1, l \geq 0$ such that $k+l=n$,

(3) $\left(T_{1}^{-1} *\right)\left(\tilde{\psi}_{n}\right)=\tilde{\psi}_{n}$,

(4) $\left[\tilde{\psi}_{n}, E_{k \delta+\alpha_{1}}\right]=\left(1+q^{-1}\right) \sum_{i=0}^{n-1} b_{2(n-i)} E_{(k+n-i) \delta+\alpha_{1}} \tilde{\psi}_{i}$ for $k \geq 0$,

(5) $\left[E_{k \delta-\alpha_{1}}, \tilde{\psi}_{n}\right]=\left(1+q^{-1}\right) \sum_{i=0}^{n-1} b_{2(n-i)} \tilde{\psi}_{i} E_{(k+n-i) \delta-\alpha_{1}}$ for $k \geq 1$,

(6) $\left[\tilde{\psi}_{n}, \tilde{\psi}_{1}\right]=0$,

(7) $\left[E_{\alpha_{0}}, E_{n \delta+\alpha_{1}}\right]_{q^{-2}}=\left(q_{1}-q_{1}^{-1}\right) \sum_{i=0}^{n} b_{2(n-i)+1} \tilde{\psi}_{i} E_{(n-i+1) \delta-\alpha_{1}}$.

Proof. We denote by $(\mathrm{a})_{r}$ the statement (a) for $n=1, \ldots, r$. We prove $(1)_{n}-(7)_{n}$ at once by the induction on $n$. If $n=1$, we have (1) and (3) by Lemma 3.8 (2) by Definition 3.3 (4) and (5) by Corollary $3.10((7),(8)),(6)$ trivially, and (7) by Lemma 3.22 with $n=1$. Now, assume that $n \geq 2$.

$(1)_{n}$ Using Lemma 3.22 and $(6)_{n-1}$, we obtain (1) for $n$.

$(2)_{n}$ Using $(1)_{n}$ and applying $T_{\varpi_{1}}^{k-1}$ to Definition 3.3, we obtain (2) for $n$.

$(3)_{n}$ By Definition 3.3, we have

$$
\left(T_{1}^{-1} *\right)\left(\tilde{\psi}_{n}\right)=\left(T_{1}^{-1} *\right)\left(\left[E_{\delta-\alpha_{1}}, E_{(n-1) \delta+\alpha_{1}}\right]_{q^{-1}}\right)=\left[E_{(n-1) \delta-\alpha_{1}}, E_{\delta+\alpha_{1}}\right]_{q^{-1}} .
$$

Applying $(2)_{n}$, we obtain (3) for $n$.

$(4)_{n}$ By $(1)_{n}$, we can assume that $k=0$. First, we consider the case where $n=2 m$ with $m \geq 1$. Note that we have $2 m>m$. Using Proposition 3.19 and Lemma 3.24, we have

$$
\begin{aligned}
& {\left[E_{\delta-\alpha_{1}},\left[E_{(2 m-1) \delta+\alpha_{1}}, E_{\alpha_{1}}\right]_{q}\right]_{q^{-2}}} \\
& =\left[E_{\delta-\alpha_{1}},(1+q) \sum_{i=1}^{m-1} b_{2 i} E_{i \delta+\alpha_{1}} E_{(2 m-i-1) \delta+\alpha_{1}} \theta(m \geq 2)\right.
\end{aligned}
$$




$$
\begin{aligned}
& \left.+[4]_{1} b_{2 m-1} E_{2 m \delta-\alpha_{0}}\right]_{q^{-2}} \\
= & (1+q) \sum_{i=1}^{m-1} b_{2 i}\left(q^{-1} E_{i \delta+\alpha_{1}} \tilde{\psi}_{2 m-i}+E_{(2 m-i-1) \delta+\alpha_{1}} \tilde{\psi}_{i+1}\right. \\
& \left.+\left[\tilde{\psi}_{i+1}, E_{(2 m-i-1) \delta+\alpha_{1}}\right]\right) \theta(m \geq 2) \\
& +[4]_{1} b_{2 m-1}\left(T_{1}^{-1} * T_{\varpi_{1}}^{m-1}\right)\left(\left[E_{\alpha_{0}}, E_{m \delta+\alpha_{1}}\right]_{q^{-2}}\right) .
\end{aligned}
$$

By $(4)_{m}$ and $(7)_{m}$, this is equal to

$$
\begin{aligned}
& \left(1+q^{-1}\right) \sum_{i=m+1}^{2 m-1} b_{2(2 m-i)} E_{(2 m-i) \delta+\alpha_{1}} \tilde{\psi}_{i} \theta(m \geq 2) \\
& +\left(1+q^{-1}\right) \sum_{i=1}^{m-1} q b_{2(i-1)} E_{(2 m-i) \delta+\alpha_{1}} \tilde{\psi}_{i} \theta(m \geq 2) \\
& +\left(1+q^{-1}\right)(1+q) \sum_{i=1}^{m-1} \sum_{j=0}^{i} b_{2 i} b_{2(i-j+1)} E_{(2 m-j) \delta+\alpha_{1}} \tilde{\psi}_{j} \theta(m \geq 2) \\
& +[4]_{1} b_{2 m-1}\left(q_{1}-q_{1}^{-1}\right)\left(T_{1}^{-1} * T_{\varpi_{1}}^{m-1}\right)\left(\sum_{i=0}^{m} b_{2(m-i)+1} \tilde{\psi}_{i} E_{(m-i+1) \delta-\alpha_{1}}\right)
\end{aligned}
$$

In the third term, putting $j=i^{\prime}, i-j+1=j^{\prime}$, we see that $i=i^{\prime}+j^{\prime}-1$ and that $1 \leq i \leq m-1,0 \leq j \leq i$ is equivalent to $0 \leq i^{\prime} \leq m-1, \max \left(1,2-i^{\prime}\right) \leq$ $j^{\prime} \leq m-i^{\prime}$. We can apply $(1)_{m}$ and $(3)_{m}$ to the fourth one. Thus, by Lemma 3.23 , we have

$$
\begin{aligned}
{\left[\tilde{\psi}_{2 m}, E_{\alpha_{1}}\right]=} & \left(1+q^{-1}\right) q b_{2} E_{2 m \delta+\alpha_{1}} \tilde{\psi}_{0}+\left(1+q^{-1}\right) q b_{0} E_{(2 m-1) \delta+\alpha_{1}} \tilde{\psi}_{1} \\
& +\left[E_{\delta-\alpha_{1}},\left[E_{(2 m-1) \delta+\alpha_{1}}, E_{\alpha_{1}}\right]_{q}\right]_{q^{-2}} \\
= & \left(1+q^{-1}\right) \sum_{i=m+1}^{2 m-1} b_{2(2 m-i)} E_{(2 m-i) \delta+\alpha_{1}} \tilde{\psi}_{i} \theta(m \geq 2) \\
& +\left(1+q^{-1}\right) \sum_{i=0}^{m}\left(q b_{2} \theta(i=0)+q b_{2(i-1)} \theta(i \geq 1)\right. \\
& +(1+q) \sum_{j=\max (1,2-i)}^{m-i} b_{2 j} b_{2(j+i-1)} \theta(m \geq 2) \theta(i \leq m-1) \\
& \left.+\left(q+q^{-1}\right)(q-1) b_{2 m-1} b_{2(m-i+1)-1}\right) E_{i \delta+\alpha_{1}} \tilde{\psi}_{2 m-i} .
\end{aligned}
$$


Applying Lemma 3.16 (2) with $l=m-i+1, s=i-1$ together with Lemma 3.14 (2), we obtain the desired result. Next, we consider the case where $n=2 m+1$ with $m \geq 1$. Note that we have $2 m+1>m+1$. Using Proposition 3.19, Lemma 3.24 and $(4)_{m+1}$, we have

$$
\begin{aligned}
& {\left[E_{\delta-\alpha_{1}},\left[E_{2 m \delta+\alpha_{1}}, E_{\alpha_{1}}\right]_{q}\right]_{q^{-2}}} \\
& =\left[E_{\delta-\alpha_{1}},(1+q) \sum_{i=1}^{m-1} b_{2 i} E_{i \delta+\alpha_{1}} E_{(2 m-i) \delta+\alpha_{1}} \theta(m \geq 2)+b_{2 m} E_{m \delta+\alpha_{1}}^{2}\right]_{q^{-2}} \\
& =(1+q) \sum_{i=1}^{m-1} b_{2 i}\left(q^{-1} E_{i \delta+\alpha_{1}} \tilde{\psi}_{2 m-i+1}+E_{(2 m-i) \delta+\alpha_{1}} \tilde{\psi}_{i+1}\right. \\
& \left.\quad+\left[\tilde{\psi}_{i+1}, E_{(2 m-i) \delta+\alpha_{1}}\right]\right) \theta(m \geq 2) \\
& \quad+b_{2 m}\left(\left(1+q^{-1}\right) E_{m \delta+\alpha_{1}} \tilde{\psi}_{m+1}+\left[\tilde{\psi}_{m+1}, E_{m \delta+\alpha_{1}}\right]\right) \\
& =\left(1+q^{-1}\right) \sum_{i=m+1}^{2 m} b_{2(2 m-i+1)} E_{(2 m-i+1) \delta+\alpha_{1}} \tilde{\psi}_{i} \\
& \quad+\left(1+q^{-1}\right) \sum_{i=2}^{m} q b_{2(i-1)} E_{(2 m-i+1) \delta+\alpha_{1}} \tilde{\psi}_{i} \theta(m \geq 2) \\
& \quad+\left(1+q^{-1}\right)(1+q) \sum_{i=1}^{m-1} \sum_{j=0}^{i} b_{2 i} b_{2(i-j+1)} E_{(2 m-j+1) \delta+\alpha_{1}} \tilde{\psi}_{j} \theta(m \geq 2) \\
& \quad+\left(1+q^{-1}\right) b_{2 m} \sum_{i=0}^{m} b_{2(m-i+1)} E_{(2 m-i+1) \delta+\alpha_{1}} \tilde{\psi}_{i} .
\end{aligned}
$$

In the third term, putting $j=i^{\prime}, i-j+1=j^{\prime}$, we see that $i=i^{\prime}+j^{\prime}-1$ and that $1 \leq i \leq m-1,0 \leq j \leq i$ is equivalent to $0 \leq i^{\prime} \leq m-1, \max \left(1,2-i^{\prime}\right) \leq$ $j^{\prime} \leq m-i^{\prime}$. Thus, by Lemma 3.23, we have

$$
\begin{aligned}
& {\left[\tilde{\psi}_{2 m+1}, E_{\alpha_{1}}\right]} \\
& =\left(1+q^{-1}\right) q b_{2} \theta(i=0)+\left(1+q^{-1}\right) q b_{2(i-1)} \theta(i \geq 1) \\
& \quad+\left[E_{\delta-\alpha_{1}},\left[E_{2 m \delta+\alpha_{1}}, E_{\alpha_{1}}\right]_{q}\right]_{q^{-2}} \\
& =\left(1+q^{-1}\right) \sum_{i=m+1}^{2 m} b_{2(2 m-i+1)} E_{(2 m-i+1) \delta+\alpha_{1}} \tilde{\psi}_{i} \\
& \quad+\left(1+q^{-1}\right) \sum_{i=0}^{m} E_{(2 m-i+1) \delta+\alpha_{1}} \tilde{\psi}_{i}\left(q b_{2} \theta(i=0)+q b_{2(i-1)} \theta(i \geq 1)\right. \\
& \left.\quad+(1+q) \sum_{j=\max (1,2-i)}^{m-i} b_{2 j} b_{2(i+j-1)} \theta(i \geq m-1) \theta(m \geq 2)+b_{2 m} b_{2(m-i+1)}\right) .
\end{aligned}
$$


Applying Lemma 3.16 (1) with $l=m-i+1, s=i-1$ together with Lemma 3.14 (1), we obtain the desired result. We obtain (4) for $n$.

$(5)_{n}$ Using $(3)_{n}$ and applying $T_{1}^{-1} *$ to $(4)_{n}$ with $k \geq 1$, we obtain (5) for $n$.

$(6)_{n}$ By Lemmas $2.21(1)$ and $3.25(2)$, it is enough to show that $r_{1}\left(\left[\tilde{\psi}_{n}\right.\right.$, $\left.\left.\tilde{\psi}_{1}\right]\right)=0$. Using Lemma 3.7 and $(1)_{n-1}$, we have $\left[E_{(n-1) \delta+\alpha_{1}}, f_{1}\right]=k_{1} \tilde{\psi}_{n-1}$. Applying Lemma 2.22 and Proposition 2.5, we have

$$
\left({ }_{1} r\left(E_{(n-1) \delta+\alpha_{1}}\right)=0 \text { and }\right) r_{1}\left(E_{(n-1) \delta+\alpha_{1}}\right)=\left(q_{1}-q_{1}^{-1}\right) \tilde{\psi}_{n-1} .
$$

Similarly, using Lemmas 3.6 ((4), (5)), 2.22, and Proposition 2.5, we have

$$
\begin{gathered}
\left({ }_{1} r\left(E_{\delta-\alpha_{1}}\right)=0 \text { and }\right) r_{1}\left(E_{\delta-\alpha_{1}}\right)=\left(q_{1}-q_{1}^{-1}\right) q^{-2}[4]_{1} E_{\alpha_{0}}, \\
\left({ }_{1} r\left(\tilde{\psi}_{1}\right)=0 \text { and }\right) r_{1}\left(\tilde{\psi}_{1}\right)=\left(q_{1}-q_{1}^{-1}\right) q^{-1}[3]_{1} ! E_{\delta-\alpha_{1}} .
\end{gathered}
$$

Hence, using the definition of $\tilde{\psi}_{n}$, we have $\left({ }_{1} r\left(\tilde{\psi}_{n}\right)=0\right.$ and $)$

$$
\begin{aligned}
r_{1}\left(\tilde{\psi}_{n}\right)= & r_{1}\left(E_{\delta-\alpha_{1}} E_{(n-1) \delta+\alpha_{1}}-q^{-1} E_{(n-1) \delta+\alpha_{1}} E_{\delta-\alpha_{1}}\right) \\
= & \left(q_{1}-q_{1}^{-1}\right)\left(q^{-1}[4]_{1} E_{\alpha_{0}} E_{(n-1) \delta+\alpha_{1}}+E_{\delta-\alpha_{1}} \tilde{\psi}_{n-1}\right) \\
& -q^{-1}\left(q_{1}-q_{1}^{-1}\right)\left(q^{-1} \tilde{\psi}_{n-1} E_{\delta-\alpha_{1}}+E_{(n-1) \delta+\alpha_{1}} q^{-2}[4]_{1} E_{\alpha_{0}}\right) \\
= & \left(q_{1}-q_{1}^{-1}\right)\left(q^{-1}[4]_{1}\left[E_{\alpha_{0}}, E_{(n-1) \delta+\alpha_{1}}\right]_{q^{-2}}\right. \\
& \left.+\left(1-q^{-2}\right) \tilde{\psi}_{n-1} E_{\delta-\alpha_{1}}+\left[E_{\delta-\alpha_{1}}, \tilde{\psi}_{n-1}\right]\right) .
\end{aligned}
$$

Applying $(5)_{n-1}$ and $(7)_{n-1}$, we have

$$
\begin{aligned}
& r_{1}\left(\tilde{\psi}_{n}\right)\left(q_{1}-q_{1}^{-1}\right)^{-1} \\
& =q^{-1}[4]_{1}\left(q_{1}-q_{1}^{-1}\right) \sum_{i=0}^{n-1} b_{2(n-i)-1} \tilde{\psi}_{i} E_{(n-i) \delta-\alpha_{1}} \\
& \quad+\left(1-q^{-2}\right) \tilde{\psi}_{n-1} E_{\delta-\alpha_{1}}+\left(1+q^{-1}\right) \sum_{i=0}^{n-2} b_{2 i} \tilde{\psi}_{i} E_{(n-i) \delta-\alpha_{1}} \\
& =\left(1+q^{-1}\right) q^{-1} \sum_{i=0}^{n-1}\left(q b_{2(n-i-1)}+\left(q+q^{-1}\right)(q-1) b_{2(n-i)-1}\right) \tilde{\psi}_{i} E_{(n-i) \delta-\alpha_{1}}
\end{aligned}
$$

Applying Lemma 3.14 (2), we have

$$
r_{1}\left(\tilde{\psi}_{n}\right)=\left(q_{1}-q_{1}^{-1}\right) q^{-1}\left(1+q^{-1}\right) \sum_{i=0}^{n-1} b_{2(n-i)} \tilde{\psi}_{i} E_{(n-i) \delta-\alpha_{1}} .
$$


Hence,

$$
\begin{aligned}
& r_{1}\left(\left[\tilde{\psi}_{n}, \tilde{\psi}_{1}\right]\right)\left(q_{1}-q_{1}^{-1}\right)^{-1} q \\
& \quad=\left(\left[r_{1}\left(\tilde{\psi}_{n}\right), \tilde{\psi}_{1}\right]+\left[\tilde{\psi}_{n}, r_{1}\left(\tilde{\psi}_{1}\right)\right]\right)\left(q_{1}-q_{1}^{-1}\right)^{-1} q \\
& \quad=\left(1+q^{-1}\right)\left[\sum_{i=0}^{n-1} b_{2(n-i)} \tilde{\psi}_{i} E_{(n-i) \delta-\alpha_{1}}, \tilde{\psi}_{1}\right]-[3]_{1} !\left[E_{\delta-\alpha_{1}}, \tilde{\psi}_{n}\right] .
\end{aligned}
$$

It follows from $(6)_{n-1}$ and Corollary 3.10 (7) that the former term is equal to

$$
\left(1+q^{-1}\right) \sum_{i=1}^{n} b_{2 i} \tilde{\psi}_{n-i} E_{(i+1) \delta-\alpha_{1}}[3]_{1} !
$$

which cancels out with the latter by $(5)_{n}$. We obtain (6) for $n$.

$(7)_{n}$ Using Lemma $3.21,(5)_{n}$ and $(7)_{n-1}$, we have

$$
\begin{aligned}
{\left[E_{\alpha_{0}}, E_{n \delta+\alpha_{1}}\right]_{q^{-2}}[3]_{1} !=} & \left(q_{1}-q_{1}^{-1}\right)[3]_{1} ! \tilde{\psi}_{n} E_{\delta-\alpha_{1}} \\
& +(q-1)[3]_{1}\left(1+q^{-1}\right) \sum_{i=0}^{n-1} b_{2(n-i)} \tilde{\psi}_{i} E_{(n-i+1) \delta-\alpha_{1}} \\
& -\left(q_{1}-q_{1}^{-1}\right)\left[\sum_{i=0}^{n-1} b_{2(n-i)-1} \tilde{\psi}_{i} E_{(n-i) \delta-\alpha_{1}}, \tilde{\psi}_{1}\right] .
\end{aligned}
$$

Applying (6) $)_{n-1}$ and Corollary 3.10 (8), we have

$$
\begin{aligned}
{\left[E_{\alpha_{0}}, E_{n \delta+\alpha_{1}}\right]_{q^{-2}}=} & \left(q_{1}-q_{1}^{-1}\right) \tilde{\psi}_{n} E_{\delta-\alpha_{1}} \\
& +\left(q_{1}-q_{1}^{-1}\right) \sum_{i=0}^{n-1}\left(b_{2(n-i)}-b_{2(n-i)-1}\right) \tilde{\psi}_{i} E_{(n-i+1) \delta-\alpha_{1}} .
\end{aligned}
$$

Applying Lemma 3.14 (1), we obtain (7) for $n$.

The proposition is proved.

Corollary 3.27. (1) Let $m, n \geq 0$. Then,

$$
\left[E_{2 m \delta+\alpha_{0}}, E_{n \delta+\alpha_{1}}\right]_{q^{-2}}=\left(q_{1}-q_{1}^{-1}\right) \sum_{i=0}^{m+n} b_{2(m+n-i)+1} \tilde{\psi}_{i} E_{(2 m+n-i+1) \delta-\alpha_{1}} .
$$

(2) Let $m, n \geq 1$. Then,

$$
\left[E_{n \delta-\alpha_{1}}, E_{2 m \delta-\alpha_{0}}\right]_{q^{-2}}=\left(q_{1}-q_{1}^{-1}\right) \sum_{i=0}^{m+n-1} b_{2(m+n-i)-1} E_{(2 m+n-i-1) \delta+\alpha_{1}} \tilde{\psi}_{i} .
$$


Proof. If $m=n=0$, then (1) is Lemma 3.6 (1). Otherwise, applying $T_{\varpi_{1}}^{m}$ to Proposition 3.26 (7) with $n$ replaced by $m+n$, we obtain (1). Applying $T_{1}^{-1} *$ to (1) with $m$ replaced by $m-1$ and with $n \geq 1$, we obtain (2).

Corollary 3.28. We have

(1) ${ }_{1} r\left(E_{\alpha}\right)=0$ for $\alpha \in R_{r e}^{+} \backslash\left\{\alpha_{1}\right\}$,

(2) ${ }_{1} r\left(\tilde{\psi}_{n}\right)=0$ for $n \geq 1$,

(3) $r_{1}\left(\tilde{\psi}_{n}\right)=\left(q_{1}-q_{1}^{-1}\right) q^{-1}\left(1+q^{-1}\right) \sum_{i=1}^{n} b_{2 i} \tilde{\psi}_{n-i} E_{i \delta-\alpha_{1}}$ for $n \geq 1$,

(4) $r_{1}\left(E_{n \delta+\alpha_{1}}\right)=\left(q_{1}-q_{1}^{-1}\right) \tilde{\psi}_{n}$ for $n \geq 0$,

(5) $r_{1}\left(E_{\delta-\alpha_{1}}\right)=\left(q_{1}-q_{1}^{-1}\right) q^{-2}[4]_{1} E_{\alpha_{0}}$.

Proof. In the proof of the induction step for Proposition 3.19 (6), we already have (1) for $\alpha=n \delta+\alpha_{1}$ with $n \geq 0$ and for $\alpha=\delta-\alpha_{1}$, and (2)-(5). By Corollary 3.10, we see that it remains to check (1) for $\alpha=2 \delta-\alpha_{0}$, but it follows from Lemma 3.9 (5).

Proposition 3.29. Let $k, l \geq 0$. Then, $\left[\tilde{\psi}_{k}, \tilde{\psi}_{l}\right]=0$.

Proof. We can assume that $1 \leq k \leq l$. We argue by the induction on $k$. By Definition 3.3 and Proposition $3.26((4),(5))$, we have

$$
\begin{aligned}
{\left[\tilde{\psi}_{k}, \tilde{\psi}_{l}\right]=} & {\left[\tilde{\psi}_{k},\left[E_{\delta-\alpha_{1}}, E_{(l-1) \delta+\alpha_{1}}\right]_{q^{-1}}\right] } \\
= & {\left[\left[\tilde{\psi}_{k}, E_{\delta-\alpha_{1}}\right], E_{(l-1) \delta+\alpha_{1}}\right]_{q^{-1}}+\left[E_{\delta-\alpha_{1}},\left[\tilde{\psi}_{k}, E_{(l-1) \delta+\alpha_{1}}\right]\right]_{q^{-1}} } \\
= & -\left(1+q^{-1}\right)\left[\sum_{i=0}^{k-1} b_{2(k-i)} \tilde{\psi}_{i} E_{(k-i+1) \delta-\alpha_{1}}, E_{(l-1) \delta+\alpha_{1}}\right]_{q^{-1}} \\
& +\left(1+q^{-1}\right)\left[E_{\delta-\alpha_{1}}, \sum_{i=0}^{k-1} b_{2(k-i)} E_{(k+l-i-1) \delta+\alpha_{1}} \tilde{\psi}_{i}\right]_{q^{-1}} .
\end{aligned}
$$

Applying Proposition 3.26 (2), we have

$$
\begin{aligned}
{\left[\tilde{\psi}_{k}, \tilde{\psi}_{l}\right]\left(1+q^{-1}\right)^{-1} } & \\
= & -\sum_{i=0}^{k-1} b_{2(k-i)}\left(\tilde{\psi}_{i}\left(q^{-1} E_{(l-1) \delta+\alpha_{1}} E_{(k-i+1) \delta-\alpha_{1}}+\tilde{\psi}_{k+l-i}\right)\right. \\
& \left.-q^{-1} E_{(l-1) \delta+\alpha_{1}} \tilde{\psi}_{i} E_{(k-i+1) \delta-\alpha_{1}}\right) \\
& +\sum_{i=0}^{k-1} b_{2(k-i)}\left(\left(q^{-1} E_{(k+l-i-1) \delta+\alpha_{1}} E_{\delta-\alpha_{1}}+\tilde{\psi}_{k+l-i}\right) \tilde{\psi}_{i}\right.
\end{aligned}
$$




$$
\begin{aligned}
& \left.-q^{-1} E_{(k+l-i-1) \delta+\alpha_{1}} \tilde{\psi}_{i} E_{\delta-\alpha_{1}}\right) \\
= & -q^{-1} \sum_{i=1}^{k-1} b_{2(k-i)}\left[\tilde{\psi}_{i}, E_{(l-1) \delta+\alpha_{1}}\right] E_{(k-i+1) \delta-\alpha_{1}} \theta(k \geq 2) \\
& +q^{-1} \sum_{i=1}^{k-1} b_{2(k-i)} E_{(k+l-i-1) \delta+\alpha_{1}}\left[E_{\delta-\alpha_{1}}, \tilde{\psi}_{i}\right] \theta(k \geq 2) \\
& +\sum_{i=0}^{k-1} b_{2(k-i)}\left[\tilde{\psi}_{k+l-i}, \tilde{\psi}_{i}\right] .
\end{aligned}
$$

By the induction hypothesis, the last term vanishes. We can assume that $k \geq 2$. Applying Proposition $3.26((4),(5))$, we have

$$
\begin{aligned}
{\left[\tilde{\psi}_{k}, \tilde{\psi}_{l}\right]\left(1+q^{-1}\right)^{-2} q=} & -\sum_{i=1}^{k-1} \sum_{j=0}^{i-1} b_{2(k-i)} b_{2(i-j)} E_{(l+i-j-1) \delta+\alpha_{1}} \tilde{\psi}_{j} E_{(k-i+1) \delta-\alpha_{1}} \\
& +\sum_{i=1}^{k-1} \sum_{j=0}^{i-1} b_{2(k-i)} b_{2(i-j)} E_{(k+l-i-1) \delta+\alpha_{1}} \tilde{\psi}_{j} E_{(i-j+1) \delta-\alpha_{1}} .
\end{aligned}
$$

In the former term, putting $k-i=i^{\prime}-j$, we see that $i=k+j-i^{\prime}$ and that $1 \leq i \leq k-1,0 \leq j \leq i-1$ is equivalent to $1 \leq i^{\prime} \leq k-1,0 \leq j \leq i^{\prime}-1$. Thus, it cancels out with the latter and the induction proceeds. The proposition is proved.

\section{$\S 4$. Construction of Basis I}

Definition 4.1. In the following, each $\mathbf{Z}_{\geq 0}^{(i)}$ is a copy of $\mathbf{Z}_{\geq 0}$.

(1) For $\mathbf{c}=\left(\mathbf{c}_{i}\right) \in \oplus_{i \in R_{r e}^{+}(>)} \mathbf{Z}_{\geq 0}^{(i)}$, we set $\mathbf{E}_{\mathbf{c}}=E_{\alpha_{1}}^{\left(\mathbf{c}_{\alpha_{1}}\right)} E_{2 \delta-\alpha_{0}}^{\left(\mathbf{c}_{2 \delta-\alpha_{0}}\right)} E_{\delta+\alpha_{1}}^{\left(\mathbf{c}_{\delta+\alpha_{1}}\right)} \cdots$

(2) For $\mathbf{c}=\left(\mathbf{c}_{i}\right) \in \oplus_{i \in R_{r e}^{+}(<)} \mathbf{Z}_{\geq 0}^{(i)}$, we set $\mathbf{E}_{\mathbf{c}}=\cdots E_{2 \delta+\alpha_{0}}^{\left(\mathbf{c}_{2 \delta+\alpha_{0}}\right)} E_{\delta-\alpha_{1}}^{\left(\mathbf{c}_{\delta-\alpha_{1}}\right)} E_{\alpha_{0}}^{\left(\mathbf{c}_{\alpha_{0}}\right)}$.

(3) For $\mathbf{c}=\left(\mathbf{c}_{i}\right) \in \oplus_{i \in \mathbf{Z}_{\geq 1}} \mathbf{Z}_{\geq 0}^{(i)}$, we set $\mathbf{E}_{\mathbf{c}}^{\prime}=\tilde{\psi}_{1}^{\mathbf{c}_{1}} \tilde{\psi}_{2}^{\mathbf{c}_{2}} \tilde{\psi}_{3}^{\mathbf{c}_{3}} \cdots$

Definition 4.2. Let $\alpha, \alpha^{\prime} \in R_{r e}^{+}(>)$and $\alpha \leq \alpha^{\prime}$. Let $\beta, \beta^{\prime} \in R_{r e}^{+}(<)$ and $\beta \leq \beta^{\prime}$. We set

(1) $B\left(>; \alpha, \alpha^{\prime}\right)=\left\{\mathbf{E}_{\mathbf{c}} \mid \mathbf{c} \in \oplus_{i \in R_{r e}^{+}(>)} \mathbf{Z}_{\geq 0}^{(i)}, \mathbf{c}_{i}=0\right.$ if $i<\alpha$ or $\left.i>\alpha^{\prime}\right\}$,

(2) $B(>; \alpha)=\left\{\mathbf{E}_{\mathbf{c}} \mid \mathbf{c} \in \oplus_{i \in R_{r e}^{+}(>)} \mathbf{Z}_{\geq 0}^{(i)}, \mathbf{c}_{i}=0\right.$ if $\left.i<\alpha\right\}$,

(3) $B\left(<; \beta, \beta^{\prime}\right)=\left\{\mathbf{E}_{\mathbf{c}} \mid \mathbf{c} \in \oplus_{i \in R_{r e}^{+}(<)} \mathbf{Z}_{\geq 0}^{(i)}, \mathbf{c}_{i}=0\right.$ if $i<\beta$ or $\left.i>\beta^{\prime}\right\}$, 
(4) $B(<; \beta)=\left\{\mathbf{E}_{\mathbf{c}} \mid \mathbf{c} \in \oplus_{i \in R_{r e}^{+}(<)} \mathbf{Z}_{\geq 0}^{(i)}, \mathbf{c}_{i}=0\right.$ if $\left.i>\beta\right\}$.

Then, each set is contained in $\mathbf{U}_{\mathbf{Z}}^{+}$and linearly independent over $\mathbf{Q}\left(q_{1}\right)[\mathrm{L}$, 40.2.1]. We also set $B(>)=B\left(>; \alpha_{1}\right)$ and $B(<)=B\left(<; \alpha_{0}\right)$.

Definition 4.3. Let $\alpha, \alpha^{\prime} \in R_{r e}^{+}(>)$and $\alpha \leq \alpha^{\prime}$.

(1) Let $\mathbf{U}^{+}\left(>; \alpha, \alpha^{\prime}\right)$ be the $\mathbf{Q}\left(q_{1}\right)$-subspace of $\mathbf{U}^{+}$with basis $B\left(>; \alpha, \alpha^{\prime}\right)$.

(2) Let $\mathbf{U}^{+}(>; \alpha)$ be the $\mathbf{Q}\left(q_{1}\right)$-subspace of $\mathbf{U}^{+}$with basis $B(>; \alpha)$. We also set $\mathbf{U}^{+}(>)=\mathbf{U}^{+}\left(>; \alpha_{1}\right)$

(3) Let $\mathbf{U}_{\mathbf{Z}}^{+}\left(>; \alpha, \alpha^{\prime}\right)$ be the free $\mathbf{Z}\left[q_{1}, q_{1}^{-1}\right]$-submodule of $\mathbf{U}_{\mathbf{Z}}^{+}$with basis $B(>$ $\left.; \alpha, \alpha^{\prime}\right)$.

(4) Let $\mathbf{U}_{\mathbf{Z}}^{+}(>; \alpha)$ be the free $\mathbf{Z}\left[q_{1}, q_{1}^{-1}\right]$-submodule of $\mathbf{U}_{\mathbf{Z}}^{+}$with basis $B(>; \alpha)$. We also set $\mathbf{U}_{\mathbf{Z}}^{+}(>)=\mathbf{U}_{\mathbf{Z}}^{+}\left(>; \alpha_{1}\right)$.

Definition 4.4. Let $\beta, \beta^{\prime} \in R_{r e}^{+}(<)$and $\beta \leq \beta^{\prime}$.

(1) Let $\mathbf{U}^{+}\left(<; \beta, \beta^{\prime}\right)$ be the $\mathbf{Q}\left(q_{1}\right)$-subspace of $\mathbf{U}^{+}$with basis $B\left(<; \beta, \beta^{\prime}\right)$.

(2) Let $\mathbf{U}^{+}(<; \beta)$ be the $\mathbf{Q}\left(q_{1}\right)$-subspace of $\mathbf{U}^{+}$with basis $B(<; \beta)$. We also set $\mathbf{U}^{+}(<)=\mathbf{U}^{+}\left(<; \alpha_{0}\right)$.

(3) Let $\mathbf{U}_{\mathbf{Z}}^{+}\left(<; \beta, \beta^{\prime}\right)$ be the free $\mathbf{Z}\left[q_{1}, q_{1}^{-1}\right]$-submodule of $\mathbf{U}_{\mathbf{Z}}^{+}$with basis $B(<$ $\left.; \beta, \beta^{\prime}\right)$.

(4) Let $\mathbf{U}_{\mathbf{Z}}^{+}(<; \beta)$ be the free $\mathbf{Z}\left[q_{1}, q_{1}^{-1}\right]$-submodule of $\mathbf{U}_{\mathbf{Z}}^{+}$with basis $B(<; \beta)$. We also set $\mathbf{U}_{\mathbf{Z}}^{+}(<)=\mathbf{U}_{\mathbf{Z}}^{+}\left(<; \alpha_{0}\right)$.

Proposition 4.5 [BCP, Proposition 2.3]. Let $\alpha \in R_{r e}^{+}(>)$and let $\beta \in$ $R_{r e}^{+}(<)$. Then, both of $\mathbf{U}_{\mathbf{Z}}^{+}\left(>; \alpha_{1}, \alpha\right)$ and $\mathbf{U}_{\mathbf{Z}}^{+}\left(<; \beta, \alpha_{0}\right)$ are closed under multiplication. In fact, for $n \geq 0$, we have

(1) $\mathbf{U}_{\mathbf{Z}}^{+}\left(>; \alpha_{1}, n \delta+\alpha_{1}\right)=\left\{x \in \mathbf{U}_{\mathbf{Z}}^{+} \mid T_{1}\left(T_{0} T_{1}\right)^{n}(x) \in \mathbf{U}_{\mathbf{Z}}^{-} \mathbf{U}_{\mathbf{Z}}^{0}\right\}$,

(2) $\mathbf{U}_{\mathbf{Z}}^{+}\left(>; \alpha_{1},(2 n+2) \delta-\alpha_{0}\right)=\left\{x \in \mathbf{U}_{\mathbf{Z}}^{+} \mid\left(T_{0} T_{1}\right)^{n+1}(x) \in \mathbf{U}_{\mathbf{Z}}^{-} \mathbf{U}_{\mathbf{Z}}^{0}\right\}$,

(3) $\mathbf{U}_{\mathbf{Z}}^{+}\left(<; 2 n \delta+\alpha_{0}, \alpha_{0}\right)=\left\{x \in \mathbf{U}_{\mathbf{Z}}^{+} \mid T_{0}^{-1}\left(T_{1}^{-1} T_{0}^{-1}\right)^{n}(x) \in \mathbf{U}_{\mathbf{Z}}^{-} \mathbf{U}_{\mathbf{Z}}^{0}\right\}$,

(4) $\mathbf{U}_{\mathbf{Z}}^{+}\left(<;(n+1) \delta-\alpha_{1}, \alpha_{0}\right)=\left\{x \in \mathbf{U}_{\mathbf{Z}}^{+} \mid\left(T_{1}^{-1} T_{0}^{-1}\right)^{n+1}(x) \in \mathbf{U}_{\mathbf{Z}}^{-} \mathbf{U}_{\mathbf{Z}}^{0}\right\}$.

Proof. Beck et al. treated the simply-laced case, but their proof of [BCP, Proposition 2.3] is applicable to our case.

Corollary 4.6. Let $\alpha, \alpha^{\prime} \in R_{r e}^{+}(>)$and $\alpha \leq \alpha^{\prime}$. Let $\beta, \beta^{\prime} \in R_{r e}^{+}(<)$and $\beta \leq \beta^{\prime}$. Then, each of the following is closed under multiplication:

(1) $\mathbf{U}_{\mathbf{Z}}^{+}\left(>; \alpha, \alpha^{\prime}\right)$, 
(2) $\mathbf{U}_{\mathbf{Z}}^{+}(>; \alpha)$,

(3) $\mathbf{U}_{\mathbf{Z}}^{+}\left(<; \beta, \beta^{\prime}\right)$,

(4) $\mathbf{U}_{\mathbf{Z}}^{+}(<; \beta)$.

Proof. For $n, m \geq 0$, we have the following isomorphisms of $\mathbf{Z}\left[q_{1}, q_{1}^{-1}\right]$ modules, which commute with the multiplication in $\mathbf{U}$ :

$$
\begin{aligned}
\mathbf{U}_{\mathbf{Z}}^{+}\left(>; \alpha_{1}, n \delta+\alpha_{1}\right) & \stackrel{T_{\varpi_{1}}^{m+1}}{\longleftarrow} \mathbf{U}_{\mathbf{Z}}^{+}\left(>;(m+1) \delta+\alpha_{1},(n+m+1) \delta+\alpha_{1}\right) \\
& \stackrel{T_{1}^{-1} *}{\longleftarrow} \mathbf{U}_{\mathbf{Z}}^{+}\left(<;(n+m+1) \delta-\alpha_{1},(m+1) \delta-\alpha_{1}\right), \\
\mathbf{U}_{\mathbf{Z}}^{+}\left(>; \alpha_{1}, 2 \delta-\alpha_{0}\right) & \stackrel{T_{\varpi_{1}}^{m+1}}{\longleftarrow} \mathbf{U}_{\mathbf{Z}}^{+}\left(>;(m+1) \delta+\alpha_{1},(2 n+2 m+4) \delta-\alpha_{0}\right) \\
& \stackrel{T_{\perp}^{T_{1}} *}{\longleftarrow} \mathbf{U}_{\mathbf{Z}}^{+}\left(<;(2 n+2 m+2) \delta+\alpha_{0},(m+1) \delta-\alpha_{1}\right), \\
\mathbf{U}_{\mathbf{Z}}^{+}\left(<; 2 n \delta+\alpha_{0}, \alpha_{0}\right) & \stackrel{T_{\varpi_{1}}^{-m}}{\longleftarrow} \mathbf{U}_{\mathbf{Z}}^{+}\left(<;(2 n+2 m) \delta+\alpha_{0}, 2 m \delta+\alpha_{0}\right) \\
& \stackrel{T_{1}^{-1} *}{\longleftarrow} \mathbf{U}_{\mathbf{Z}}^{+}\left(>;(2 m+2) \delta-\alpha_{0},(2 n+2 m+2) \delta-\alpha_{0}\right), \\
\mathbf{U}_{\mathbf{Z}}^{+}\left(<;(n+1) \delta-\alpha_{1}, \alpha_{0}\right) & \stackrel{T_{\varpi_{1}}^{-m}}{\longleftarrow} \mathbf{U}_{\mathbf{Z}}^{+}\left(<;(n+m+1) \delta-\alpha_{1}, 2 m \delta+\alpha_{0}\right) \\
& \stackrel{T_{1}^{-1} *}{\longleftarrow} \mathbf{U}_{\mathbf{Z}}^{+}\left(>;(2 m+2) \delta-\alpha_{0},(n+m+1) \delta+\alpha_{1}\right) .
\end{aligned}
$$

(1) and (3) follow from Proposition 4.5 by using the above morphisms. (2) and (4) follow from (1) and (3) respectively.

Corollary 4.7. Let $\alpha, \alpha^{\prime} \in R_{r e}^{+}(>)$and let $\beta, \beta^{\prime} \in R_{r e}^{+}(<)$. Then, each of the following is closed under multiplication:

(1) $\mathbf{U}^{+}\left(>; \alpha, \alpha^{\prime}\right)$,

(2) $\mathbf{U}^{+}(>; \alpha)$,

(3) $\mathbf{U}^{+}\left(<; \beta, \beta^{\prime}\right)$,

(4) $\mathbf{U}^{+}(<; \beta)$.

Proof. This follows from Corollary 4.6.

Definition 4.8. Let $n \geq 0$.

(1) Let $\mathbf{U}^{+}(0)$ be the $\mathbf{Q}\left(q_{1}\right)$-subalgebra of $\mathbf{U}^{+}$generated by $\tilde{\psi}_{i}$ for $i \geq 1$.

(2) Let $\mathbf{U}^{+}(0 ; n)$ be the $\mathbf{Q}\left(q_{1}\right)$-subalgebra of $\mathbf{U}^{+}$generated by $\tilde{\psi}_{i}$ for $1 \leq i \leq n$.

We understand that $\mathbf{U}^{+}(0 ; 0)=\mathbf{Q}\left(q_{1}\right)$. 
We introduce new imaginary root vectors.

Definition 4.9. For $n \geq 0$, we define the elements $P_{n}$ of $\mathbf{U}^{+}(0 ; n)$ by the induction on $n$ as follows: we set $P_{0}=1$ and

$$
P_{n}=[2 n]_{1}^{-1} \sum_{k=0}^{n-1} P_{k} \tilde{\psi}_{n-k} q^{-k} \quad \text { for } n \geq 1 \text {. }
$$

Also, for $\mathbf{c}=\left(\mathbf{c}_{i}\right) \in \oplus_{i \in \mathbf{Z}_{\geq 1}} \mathbf{Z}_{\geq 0}^{(i)}$, we set $\mathbf{E}_{\mathbf{c}}=P_{1}^{\mathbf{c}_{1}} P_{2}^{\mathbf{c}_{2}} P_{3}^{\mathbf{c}_{3}} \cdots$.

Lemma 4.10. Let $n, m \geq 0$. Then,

(1) $\left[P_{n}, P_{m}\right]=0$,

(2) $T_{\varpi_{1}}\left(P_{n}\right)=P_{n}$,

(3) $T_{1}^{-1} *\left(P_{n}\right)=P_{n}$.

Proof. This follows from Propositions 3.29 and 3.26.

Lemma 4.11. Let $n \geq 1$. Then,

$$
\tilde{\psi}_{n}=(-1)^{n-1} q^{-n}\left|\begin{array}{cccc}
\alpha_{1} & 1 & 0 & 0 \\
\alpha_{2} & P_{1} & \ddots & 0 \\
\vdots & \vdots & \ddots & 1 \\
\alpha_{n} & P_{n-1} & \cdots & P_{1}
\end{array}\right|
$$

where we set $\alpha_{k}=P_{k}[2 k]_{1} q^{k}$ for $1 \leq k \leq n$.

Proof. We argue by the induction on $n$. The case where $n=1$ is clear: $\tilde{\psi}_{1}=P_{1}[2]_{1}$. Assume that $n \geq 2$. Then,

$$
\begin{aligned}
\left|\begin{array}{cccc}
\alpha_{1} & 1 & 0 & 0 \\
\alpha_{2} & P_{1} & \ddots & 0 \\
\vdots & \vdots & \ddots & 1 \\
\alpha_{n} & P_{n-1} & \cdots & P_{1}
\end{array}\right| & =\sum_{k=1}^{n-1}(-1)^{k+1} P_{k}\left|\begin{array}{cccc}
\alpha_{1} & 1 & 0 & 0 \\
\alpha_{2} & P_{1} & \ddots & 0 \\
\vdots & \vdots & \ddots & 1 \\
\alpha_{n-k} & P_{n-k-1} & \cdots & P_{1}
\end{array}\right| \\
& -(-q)^{n}[2 n]_{1} P_{n},
\end{aligned}
$$

which is checked by developing the left hand side with respect to the last row. Using the induction hypothesis, we see that this is equal to

$$
(-q)^{n}\left(\sum_{k=1}^{n-1} P_{k} \tilde{\psi}_{n-k} q^{-k}-[2 n]_{1} P_{n}\right) .
$$


On the other hand, it follows from Definition 4.9 that

$$
\tilde{\psi}_{n}=[2 n]_{1} P_{n}-\sum_{k=1}^{n-1} P_{k} \tilde{\psi}_{n-k} q^{-k} .
$$

Thus, the induction proceeds.

Corollary 4.12. Let $n \geq 0$. Then, $\mathbf{U}^{+}(0 ; n)$ is generated by $P_{k}$ for $0 \leq k \leq n$.

Proof. This follows from Lemma 4.11.

Remark 4.13. Lemma 4.11 suggests that $P_{n}$ and $\tilde{\psi}_{n}$ are $q$-analogues of the complete symmetric function and two times of the power sum respectively (see [M, 1.2.ex.8]).

Remark 4.14. If we define $E_{n \delta} \in \mathbf{U}^{+}(0 ; n)$ for $n \geq 1$ as in Appendix B, then we have

$$
\sum_{k \geq 0} P_{k} u^{k}=\exp \left(\sum_{n \geq 1}[2 n]_{1}^{-1} E_{n \delta} u^{n}\right) .
$$

Let us study the commutation relations between $P_{n}$ and the real root vectors.

Lemma 4.15. Let $n \geq 0$. Then,

$$
P_{n} E_{m \delta+\alpha_{1}}=\sum_{i=0}^{n} E_{(m+n-i) \delta+\alpha_{1}} P_{i}[2 n-2 i+1]_{1} .
$$

Proof. We argue by the induction on $n$. We can assume that $n \geq 1$. By virtue of Lemma 4.10 (2), we can assume that $m=0$. By Definition 4.9 and Proposition 3.26 (5), we have

$$
\begin{aligned}
& P_{n} E_{\alpha_{1}}[2 n]_{1} \\
& =\sum_{k=0}^{n-1} P_{k} \tilde{\psi}_{n-k} E_{\alpha_{1}} q^{-k} \\
& =\sum_{k=0}^{n-1} P_{k}\left(E_{\alpha_{1}} \tilde{\psi}_{n-k}+\left(1+q^{-1}\right) \sum_{i=0}^{n-k-1} b_{2(n-k-i)} E_{(n-k-i) \delta+\alpha_{1}} \tilde{\psi}_{i}\right) q^{-k} .
\end{aligned}
$$


Using the induction hypothesis, we see that this is equal to

$$
\begin{aligned}
& \sum_{k=0}^{n-1} \sum_{i=0}^{k} E_{(k-i) \delta+\alpha_{1}} P_{i} \tilde{\psi}_{n-k}[2 k-2 i+1]_{1} q^{-k} \\
& +\left(1+q^{-1}\right) \sum_{k=0}^{n-1} \sum_{i=0}^{n-k-1} \sum_{j=0}^{k} E_{(n-i-j) \delta+\alpha_{1}} P_{j} \tilde{\psi}_{i} b_{2(n-k-i)}[2 k-2 j+1]_{1} q^{-k} .
\end{aligned}
$$

In the former term, putting $k-i=n-i^{\prime}, i=j^{\prime}$, we see that $k=n-i^{\prime}+j^{\prime}$ and that $0 \leq k \leq n-1,0 \leq i \leq k$ is equivalent to $1 \leq i^{\prime} \leq n, 0 \leq j^{\prime} \leq i^{\prime}-1$. In the latter, putting $i+j=i^{\prime}, n-k-i=k^{\prime}$, we see that $i=i^{\prime}-j, k=n-i^{\prime}+j-k^{\prime}$ and that $0 \leq k \leq n-1,0 \leq i \leq n-k-1,0 \leq j \leq k$ is equivalent to $0 \leq i^{\prime} \leq n-1,0 \leq j \leq i^{\prime}, 1 \leq k^{\prime} \leq n-i^{\prime}$. Hence, using Lemma 3.17, we have

$$
\begin{aligned}
& P_{n} E_{\alpha_{1}}[2 n]_{1} \\
& =\sum_{i=1}^{n} \sum_{j=0}^{i-1} E_{(n-i) \delta+\alpha_{1}} P_{j} \tilde{\psi}_{i-j}[2 n-2 i+1]_{1} q^{-n+i-j} \\
& \quad+\left(1+q^{-1}\right) \sum_{i=0}^{n-1} \sum_{j=0}^{i} \sum_{k=1}^{n-i} E_{(n-i) \delta+\alpha_{1}} P_{j} \tilde{\psi}_{i-j} b_{2 k}[2 n-2 i-2 k+1]_{1} q^{-n+i-j+k} \\
& =\sum_{i=1}^{n} \sum_{j=0}^{i-1} E_{(n-i) \delta+\alpha_{1}} P_{j} \tilde{\psi}_{i-j}[2 n-2 i+1]_{1} q^{-n+i-j} \\
& \quad+\sum_{i=0}^{n-1} \sum_{j=0}^{i} E_{(n-i) \delta+\alpha_{1}} P_{j} \tilde{\psi}_{i-j}[2 n-2 i+1]_{1}\left(q^{n-i}-q^{-n+i}\right) q^{-j} .
\end{aligned}
$$

In the latter term, on account of the factor $\left(q^{n-i}-q^{-n+i}\right)$, we can include the case for $i=n$. Thus, using Definition 4.9, we have

$$
\begin{aligned}
P_{n} E_{\alpha_{1}}[2 n]_{1}= & \sum_{i=1}^{n} \sum_{j=0}^{i-1} E_{(n-i) \delta+\alpha_{1}} P_{j} \tilde{\psi}_{i-j}[2 n-2 i+1]_{1} q^{n-i-j} \\
& +\sum_{i=0}^{n} E_{(n-i) \delta+\alpha_{1}} P_{i} \tilde{\psi}_{0}[2 n-2 i+1]_{1}\left(q^{n-i}-q^{-n+i}\right) q^{-i} \\
= & \sum_{i=0}^{n} E_{(n-i) \delta+\alpha_{1}} P_{i}[2 n-2 i+1]_{1}\left([2 i]_{1} q^{n-i}+[2 n-2 i]_{1} q^{-i}\right) \\
= & \sum_{i=0}^{n} E_{(n-i) \delta+\alpha_{1}} P_{i}[2 n-2 i+1]_{1}[2 n]_{1} .
\end{aligned}
$$

Thus, the induction proceeds. 
Lemma 4.16. Let $n \geq 1, m \geq 0$. Then,

(1) $\left[P_{n}, E_{(2 m+2) \delta-\alpha_{0}}\right]=\sum_{i=0}^{n-1} x_{i} P_{i}$ for some $x_{i} \in \mathbf{U}^{+, h}\left(>;(m+1) \delta+\alpha_{1}\right)$ with $\mathrm{h}\left(x_{i}\right)=2$,

(2) $\left[E_{2 m \delta+\alpha_{0}}, P_{n}\right]=\sum_{i=0}^{n-1} P_{i} z_{i}$ for some $z_{i} \in \mathbf{U}^{+, h}\left(<;(m+1) \delta-\alpha_{1}\right)$ with $\mathrm{h}\left(z_{i}\right)=-2$.

Proof. By using $T_{1}^{-1} *,(2)$ is reduced to (1). By using $T_{\varpi_{1}}^{m},(1)$ is reduced to the case where $m=0$, which we shall check now. By Corollary 3.10 (5) and Lemma 4.15, we have

$$
\begin{aligned}
P_{n} & E_{2 \delta-\alpha_{0}}[4]_{1} \\
= & P_{n}\left[E_{\delta+\alpha_{1}}, E_{\alpha_{1}}\right]_{q} \\
= & \sum_{i=0}^{n} E_{(n-i+1) \delta+\alpha_{1}} P_{i}[2 n-2 i+1]_{1} E_{\alpha_{1}} \\
& \quad-q \sum_{i=0}^{n} E_{(n-i) \delta+\alpha_{1}} P_{i}[2 n-2 i+1]_{1} E_{\delta+\alpha_{1}} \\
= & \sum_{i=0}^{n} \sum_{j=0}^{i} E_{(n-i+1) \delta+\alpha_{1}} E_{(i-j) \delta+\alpha_{1}} P_{j}[2 n-2 i+1]_{1}[2 i-2 j+1]_{1} \\
& -q \sum_{i=0}^{n} \sum_{j=0}^{i} E_{(n-i) \delta+\alpha_{1}} E_{(i-j+1) \delta+\alpha_{1}} P_{j}[2 n-2 i+1]_{1}[2 i-2 j+1]_{1} .
\end{aligned}
$$

The $j \leq i-1$ part in the former term plus the $i \leq n-1$ part in the latter can be written in the desired form (i.e. as in the right hand side of the statement) by Corollary 3.20. The remainder is equal to

$$
\begin{aligned}
& \sum_{i=0}^{n} E_{(n-i+1) \delta+\alpha_{1}} E_{\alpha_{1}} P_{i}[2 n-2 i+1]_{1} \\
& -q \sum_{j=0}^{n} E_{\alpha_{1}} E_{(n-j+1) \delta+\alpha_{1}} P_{j}[2 n-2 j+1]_{1} \\
& \quad=\sum_{i=0}^{n}\left[E_{(n-i+1) \delta+\alpha_{1}}, E_{\alpha_{1}}\right]_{q} P_{i}[2 n-2 i+1]_{1} .
\end{aligned}
$$

The $i=n$ part is equal to $E_{2 \delta-\alpha_{0}} P_{n}[4]_{1}$ by Corollary $3.10(5)$. The $i \leq n-1$ part can be written in the desired form by Proposition 3.19. Thus, we obtain the lemma.

Proposition 4.17. Let $n \geq 0$ and let $\alpha \in R_{r e}^{+}(>), \beta \in R_{r e}^{+}(<)$. 
(1) Let $x \in \mathbf{U}^{+, h}(>; \alpha), y \in \mathbf{U}^{+, h}(0 ; n)$. Then, $y x=\sum_{i} x_{i} y_{i}$ for some $x_{i} \in$ $\mathbf{U}^{+, h}(>; \alpha), y_{i} \in \mathbf{U}^{+, h}(0 ; n)$ with $\mathrm{h}\left(x_{i}\right)=\mathrm{h}(x), \mathrm{i}\left(y_{i}\right) \leq \mathrm{i}(y)$.

(2) $\mathbf{U}^{+}(>; \alpha) \mathbf{U}^{+}(0 ; n)$ is closed under multiplication.

(3) Let $y \in \mathbf{U}^{+, h}(0 ; n), z \in \mathbf{U}^{+, h}(<; \beta)$. Then, $z y=\sum_{i} y_{i} z_{i}$ for some $y_{i} \in$ $\mathbf{U}^{+, h}(0 ; n), z_{i} \in \mathbf{U}^{+, h}(<; \beta)$ with $\mathrm{i}\left(y_{i}\right) \leq \mathrm{i}(y), \mathrm{h}\left(z_{i}\right)=\mathrm{h}(z)$.

(4) $\mathbf{U}^{+}(0 ; n) \mathbf{U}^{+}(<; \beta)$ is closed under multiplication.

Proof. We prove (1) by the induction on $\mathrm{h}(x)+\mathrm{i}(y)$. We can assume that $\mathrm{h}(x) \geq 1$ and $\mathrm{i}(y) \geq 1$. First, assume that $x=x^{\prime} x^{\prime \prime}$ for some $x^{\prime}, x^{\prime \prime} \in \mathbf{U}^{+, h}(>)$ with $\mathrm{h}\left(x^{\prime}\right)<\mathrm{h}(x), \mathrm{h}\left(x^{\prime \prime}\right)<\mathrm{h}(x)$. Since $\mathrm{h}\left(x^{\prime}\right)+\mathrm{i}(y)<\mathrm{h}(x)+\mathrm{i}(y)$, by the induction hypothesis, we have $y x=y x^{\prime} x^{\prime \prime}=\sum x_{1} y_{1} x^{\prime \prime}$ for some $x_{1} \in \mathbf{U}^{+, h}(>$ $; \alpha), y_{1} \in \mathbf{U}^{+, h}(0 ; n)$ with $\mathrm{h}\left(x_{1}\right)=\mathrm{h}\left(x^{\prime}\right), \mathrm{i}\left(y_{1}\right) \leq \mathrm{i}(y)$. Since $\mathrm{h}\left(x^{\prime \prime}\right)+\mathrm{i}\left(y_{1}\right)<$ $\mathrm{h}(x)+\mathrm{i}(y)$, by the induction hypothesis, we have $y x=\sum x_{1} x_{2} y_{2}$ for some $x_{2} \in$ $\mathbf{U}^{+, h}(>; \alpha), y_{2} \in \mathbf{U}^{+, h}(0 ; n)$ with $\mathrm{h}\left(x_{2}\right)=\mathrm{h}\left(x^{\prime \prime}\right), \mathrm{i}\left(y_{2}\right) \leq \mathrm{i}\left(y_{1}\right)$. By Corollary 4.7, we have $x_{1} x_{2} \in \mathbf{U}^{+, h}(>; \alpha)$. We also have $\mathrm{h}\left(x_{1} x_{2}\right)=\mathrm{h}\left(x^{\prime}\right)+\mathrm{h}\left(x^{\prime \prime}\right)=\mathrm{h}(x)$ and $\mathrm{i}\left(y_{2}\right) \leq \mathrm{i}(y)$. Thus, (1) is reduced to the case where $x=E_{k \delta+\alpha_{1}}$ with $k \delta+\alpha_{1} \geq \alpha$ or $E_{(2 k+2) \delta-\alpha_{0}}$ with $(2 k+2) \delta-\alpha_{0} \geq \alpha$. Similarly, we can also assume that $y=P_{i}$ for $1 \leq i \leq n$. Thus, (1) follows from Lemmas 4.15 and 4.16 .

We prove (2). Let $x, x^{\prime} \in \mathbf{U}^{+, h}(>; \alpha), y, y^{\prime} \in \mathbf{U}^{+, h}(0 ; n)$. By (1), we have $x y x^{\prime} y^{\prime}=x x_{1} y_{1} y^{\prime}$ for some $x_{1} \in \mathbf{U}^{+, h}(>; \alpha), y_{1} \in \mathbf{U}^{+, h}(0 ; n)$. Thus, (2) follows from Corollary 4.7.

Applying $T_{1}^{-1} *$ to (1) and (2), we obtain (3) and (4) respectively.

Remark 4.18. In the above proof of (1), we understand that each suffix of $x, y$ indicates the number of real suffices: $x_{1}=x_{i}, y_{1}=y_{i}, x_{2}=x_{i j}, y_{2}=y_{i j}$ where $i, j$ belong to some finite sets $I_{1}, I_{2}$ respectively. We shall sometimes use such a notation.

Proposition 4.19. Let $\alpha \in R_{r e}^{+}(>), \beta \in R_{r e}^{+}(<)$.

(1) Let $x \in \mathbf{U}^{+, h}(>; \alpha), z \in \mathbf{U}^{+, h}(<; \beta)$. Then, $z x=\sum_{i} x_{i} y_{i} z_{i}$ for some $x_{i} \in$ $\mathbf{U}^{+, h}(>; \alpha), y_{i} \in \mathbf{U}^{+, h}(0), z_{i} \in \mathbf{U}^{+, h}(<; \beta)$ with $\mathrm{h}\left(x_{i}\right) \leq \mathrm{h}(x), \mathrm{h}\left(z_{i}\right) \geq$ $\mathrm{h}(z)$.

(2) $\mathbf{U}^{+}(>; \alpha) \mathbf{U}^{+}(0) \mathbf{U}^{+}(<; \beta)$ is closed under multiplication.

Proof. (1) We argue by the induction on $\mathrm{h}(x)-\mathrm{h}(z)$. If $\mathrm{h}(x)=0$ or $\mathrm{h}(z)=$ 0 , the statement is clear. We assume that $\mathrm{h}(x) \geq 1$ and $\mathrm{h}(z) \leq-1$. First, assume that $x=x^{\prime} x^{\prime \prime}$ with $x^{\prime}, x^{\prime \prime} \in \mathbf{U}^{+, h}(>)$ and $\mathrm{h}\left(x^{\prime}\right)<\mathrm{h}(x), \mathrm{h}\left(x^{\prime \prime}\right)<\mathrm{h}(x)$. 
Since $\mathrm{h}\left(x^{\prime}\right)-\mathrm{h}(z)<\mathrm{h}(x)-\mathrm{h}(z)$, by the induction hypothesis, we have $z x=$ $z x^{\prime} x^{\prime \prime}=\sum x_{1} y_{1} z_{1} x^{\prime \prime}$ for some $x_{1} \in \mathbf{U}^{+, h}(>; \alpha), y_{1} \in \mathbf{U}^{+, h}(0), z_{1} \in \mathbf{U}^{+, h}(<; \beta)$ with $\mathrm{h}\left(x_{1}\right) \leq \mathrm{h}\left(x^{\prime}\right), \mathrm{h}\left(z_{1}\right) \geq \mathrm{h}(z)$. Since $\mathrm{h}\left(x^{\prime \prime}\right)-\mathrm{h}\left(z_{1}\right)<\mathrm{h}(x)-\mathrm{h}(z)$, by the induction hypothesis, we have $z x=\sum x_{1} y_{1} x_{2} y_{2} z_{2}$ for some $x_{2} \in \mathbf{U}^{+, h}(>$ $; \alpha), y_{2} \in \mathbf{U}^{+, h}(0), z_{2} \in \mathbf{U}^{+, h}(<; \beta)$ with $\mathrm{h}\left(x_{2}\right) \leq \mathrm{h}\left(x^{\prime \prime}\right), \mathrm{h}\left(z_{2}\right) \geq \mathrm{h}\left(z_{1}\right)$. By Proposition 4.17 (1), we have $z x=\sum x_{1} x_{3} y_{3} y_{2} z_{2}$ for some $x_{3} \in \mathbf{U}^{+, h}(>$ $; \alpha), y_{3} \in \mathbf{U}^{+, h}(0)$ with $\mathrm{h}\left(x_{3}\right)=\mathrm{h}\left(x_{2}\right)$. Then, $x_{1} x_{3} \in \mathbf{U}^{+, h}(>; \alpha), y_{3} y_{2} \in$ $\mathrm{U}^{+, h}(0)$ with $\mathrm{h}\left(x_{1} x_{3}\right) \leq \mathrm{h}\left(x^{\prime}\right)+\mathrm{h}\left(x^{\prime \prime}\right)=\mathrm{h}(x), \mathrm{h}\left(z_{2}\right) \geq \mathrm{h}(z)$. Thus, (1) is reduced to the case where $x=E_{k \delta+\alpha_{1}}$ with $k \delta+\alpha_{1} \geq \alpha$ or $x=E_{2 k \delta-\alpha_{0}}$ with $2 k \delta-\alpha_{0} \geq \alpha$. Similarly, we can also assume that $z=E_{k \delta-\alpha_{1}}$ with $k \delta-\alpha_{1} \leq \beta$ or $z=E_{2 k \delta+\alpha_{0}}$ with $2 k \delta+\alpha_{0} \leq \beta$. Now, (1) follows from Proposition 3.26 (2), Corollary 3.27 and Corollary A.3, which will be shown in Appendix A.

(2) Let $x, x^{\prime} \in \mathbf{U}_{\mathbf{Z}}^{+, h}(>; \alpha), y, y^{\prime} \in \mathbf{U}_{\mathbf{Z}}^{+, h}(0), z, z^{\prime} \in \mathbf{U}_{\mathbf{Z}}^{+, h}(<; \beta)$. By (1) and Proposition 4.17, we have $x y z x^{\prime} y^{\prime} z^{\prime}=x y x_{1} y_{1} z_{1} y^{\prime} z^{\prime}=x x_{2} y_{2} y_{1} y_{2}^{\prime} z_{2} z^{\prime}$ for some $x_{1}, x_{2} \in \mathbf{U}_{\mathbf{Z}}^{+, h}(>; \alpha), y_{1}, y_{2}, y_{2}^{\prime} \in \mathbf{U}_{\mathbf{Z}}^{+, h}(0), z_{1}, z_{2} \in \mathbf{U}_{\mathbf{Z}}^{+, h}(<; \beta)$. Hence, (2) follows from Corollary 4.7.

Corollary 4.20. The $\mathbf{Q}\left(q_{1}\right)$-linear map $\mathbf{U}^{+}(>) \otimes \mathbf{U}^{+}(0) \otimes \mathbf{U}^{+}(<) \longrightarrow$ $\mathbf{U}^{+}$given by multiplication is surjective.

Proof. Let $V=\mathbf{U}^{+}(>) \mathbf{U}^{+}(0) \mathbf{U}^{+}(<)$be the image of the above morphism. Since $V$ contains the generators of the $\mathbf{Q}\left(q_{1}\right)$-algebra $\mathbf{U}^{+}$, it follows from Proposition 4.19 (2) that $V=\mathbf{U}^{+}$.

In Section 6, we shall prove that the above morphism is an isomorphism and that both of $\left\{P_{n} \mid n \geq 1\right\}$ and $\left\{\tilde{\psi}_{n} \mid n \geq 1\right\}$ are algebraically independent over $\mathbf{Q}\left(q_{1}\right)$; thus, we shall obtain $\mathbf{Q}\left(q_{1}\right)$-bases of $\mathbf{U}^{+}$.

\section{$\S 5 . \quad$ Lemmas on Coproduct}

In this section, $\equiv$ means the congruence modulo $\mathbf{U}^{0} \mathbf{U}^{+}(<)_{\leq-2} \otimes \mathbf{U}^{+}$, unless otherwise stated.

Lemma 5.1. We have

$$
\begin{aligned}
\Delta\left(E_{\delta-\alpha_{1}}\right)= & c k_{1}^{-1} \otimes E_{\delta-\alpha_{1}}+E_{\delta-\alpha_{1}} \otimes 1+\left(q^{2}-q^{-2}\right) k_{1} E_{\alpha_{0}} \otimes E_{\alpha_{1}}, \\
\Delta\left(\tilde{\psi}_{1}\right)= & c \otimes \tilde{\psi}_{1}+\tilde{\psi}_{1} \otimes 1+\left(q-q^{-1}\right)[3]_{1} k_{1} E_{\delta-\alpha_{1}} \otimes E_{\alpha_{1}} \\
& +\left(1-q^{-3}\right)\left(q^{4}-1\right) k_{1}^{2} E_{\alpha_{0}} \otimes E_{\alpha_{1}}^{2} .
\end{aligned}
$$

Proof. (1) By Lemma 3.6 (1), we have

$$
\Delta\left(E_{\delta-\alpha_{1}}\right)=\Delta\left(\left[E_{\alpha_{0}}, E_{\alpha_{1}}\right]_{q^{-2}}\right)
$$




$$
\begin{aligned}
= & {\left[E_{\alpha_{0}} \otimes 1+k_{0} \otimes E_{\alpha_{0}}, E_{\alpha_{1}} \otimes 1+k_{1} \otimes E_{\alpha_{1}}\right]_{q^{-2}} } \\
= & E_{\delta-\alpha_{1}} \otimes 1+\left(k_{0} E_{\alpha_{1}}-q^{-2} E_{\alpha_{1}} k_{0}\right) \otimes E_{\alpha_{0}} \\
& +\left(E_{\alpha_{0}} k_{1}-q^{-2} k_{1} E_{\alpha_{0}}\right) \otimes E_{\alpha_{1}}+c k_{1}^{-1} \otimes E_{\delta-\alpha_{1}} .
\end{aligned}
$$

The second term vanishes and we obtain (1).

(2) By Definition 3.3 and (1), we have

$$
\begin{aligned}
\Delta\left(\tilde{\psi}_{1}\right)= & \Delta\left(\left[E_{\delta-\alpha_{1}}, E_{\alpha_{1}}\right]_{q^{-1}}\right) \\
= & {\left[c k_{1}^{-1} \otimes E_{\delta-\alpha_{1}}+E_{\delta-\alpha_{1}} \otimes 1+\left(q^{2}-q^{-2}\right) k_{1} E_{\alpha_{0}} \otimes E_{\alpha_{1}}\right.} \\
& \left.k_{1} \otimes E_{\alpha_{1}}+E_{\alpha_{1}} \otimes 1\right]_{q^{-1}} \\
= & c \otimes \tilde{\psi}_{1}+\left(E_{\delta-\alpha_{1}} k_{1}-q^{-1} k_{1} E_{\delta-\alpha_{1}}\right) \otimes E_{\alpha_{1}} \\
& +\left(q^{2}-q^{-2}\right)\left(k_{1} E_{\alpha_{0}} k_{1}-q^{-1} k_{1}^{2} E_{\alpha_{0}}\right) \otimes E_{\alpha_{1}}^{2} \\
& +c\left(k_{1}^{-1} E_{\alpha_{1}}-q^{-1} E_{\alpha_{1}} k_{1}^{-1}\right) \otimes E_{\delta-\alpha_{1}}+\tilde{\psi}_{1} \otimes 1 \\
& +\left(q^{2}-q^{-2}\right)\left(k_{1} E_{\alpha_{0}} E_{\alpha_{1}}-q^{-1} E_{\alpha_{1}} k_{1} E_{\alpha_{0}}\right) \otimes E_{\alpha_{1}} \\
= & c \otimes \tilde{\psi}_{1}+\tilde{\psi}_{1} \otimes 1+\left(\left(q-q^{-1}\right)+\left(q^{2}-q^{-2}\right)\right) k_{1} E_{\delta-\alpha_{1}} \otimes E_{\alpha_{1}} \\
& +\left(q^{2}-q^{-2}\right)\left(q^{2}-q^{-1}\right) k_{1}^{2} E_{\alpha_{0}} \otimes E_{\alpha_{1}}^{2} .
\end{aligned}
$$

Thus, we obtain (2).

Lemma 5.2. Let $n, r \geq 1$, let $\beta \in R_{r e}^{+}(<)$, and let $x \in \mathbf{U}^{+}(<; \beta) \cap$ $\mathbf{U}_{n \delta-r \alpha_{1}}^{+}$. Then, $\left[x, \tilde{\psi}_{1}\right] \in \mathbf{U}^{+}(<; \beta)$.

Proof. We argue by the induction on $r$. First, assume that $x=x_{1} x_{2}$ for some $x_{i} \in \mathbf{U}^{+}(<; \beta) \cap \mathbf{U}_{n_{i} \delta-r_{i} \alpha_{1}}^{+}$with $r_{i} \geq 1$. Then,

$$
\left[x, \tilde{\psi}_{1}\right]=\left[x_{1} x_{2}, \tilde{\psi}_{1}\right]=x_{1}\left[x_{2}, \tilde{\psi}_{1}\right]+\left[x_{1}, \tilde{\psi}_{1}\right] x_{2}
$$

and this belongs to $\mathbf{U}^{+}(<; \beta)$ by the induction hypothesis and Corollary 4.7. Thus, the lemma is reduced to the case where $x=E_{n \delta-\alpha_{1}}$ or $x=E_{2 m \delta+\alpha_{0}}$ with $m \geq 0$, but it follows from Corollary 3.10.

Lemma 5.3. Let $n \geq 1$. Then,

$$
\Delta\left(E_{n \delta-\alpha_{1}}\right) \equiv c^{n} k_{1}^{-1} \otimes E_{n \delta-\alpha_{1}}+\left(q_{1}-q_{1}^{-1}\right) \sum_{i=1}^{n} c^{n-i} E_{i \delta-\alpha_{1}} \otimes \tilde{\psi}_{n-i} .
$$

Proof. We argue by the induction on $n$. The case where $n=1$ follows from Lemma 5.1 (1). Assuming the case for $n$, we shall prove the case for $n+1$. 
Using Corollary 3.10, the induction hypothesis, and Lemma 5.1 (2), in view of Proposition 4.7, we have

$$
\begin{aligned}
\Delta\left(E_{(n+1) \delta-\alpha_{1}}\right)[3]_{1} != & \Delta\left(\left[E_{n \delta-\alpha_{1}}, \tilde{\psi}_{1}\right]\right) \\
\equiv & {\left[c^{n} k_{1}^{-1} \otimes E_{n \delta-\alpha_{1}}+\left(q_{1}-q_{1}^{-1}\right) \sum_{i=1}^{n} c^{n-i} E_{i \delta-\alpha_{1}} \otimes \tilde{\psi}_{n-i}\right.} \\
& \left.c \otimes \tilde{\psi}_{1}+\tilde{\psi}_{1} \otimes 1+\left(q-q^{-1}\right)[3]_{1} k_{1} E_{\delta-\alpha_{1}} \otimes E_{\alpha_{1}}\right] \\
\equiv & c^{n+1} k_{1}^{-1} \otimes E_{(n+1) \delta-\alpha_{1}}[3]_{1} ! \\
& +\left(q_{1}-q_{1}^{-1}\right) \sum_{i=1}^{n} c^{n-i} E_{(i+1) \delta-\alpha_{1}}[3]_{1} ! \otimes \tilde{\psi}_{n-i} \\
& +\left(q-q^{-1}\right)[3]_{1} c^{n} E_{\delta-\alpha_{1}} \otimes\left[E_{n \delta-\alpha_{1}}, E_{\alpha_{1}}\right]_{q^{-1}} .
\end{aligned}
$$

Applying Proposition 3.26 to the last term, we obtain the case for $n+1$.

Lemma 5.4. Let $n \geq 0$. Then,

$$
\begin{aligned}
\Delta\left(E_{2 n \delta+\alpha_{0}}\right) \equiv & c^{2 n+1} k_{1}^{-2} \otimes E_{2 n \delta+\alpha_{0}} \\
& +\left(q_{1}-q_{1}^{-1}\right)^{2} \sum_{i=1}^{n} c^{2 n-i+1} k_{1}^{-1} E_{i \delta-\alpha_{1}} \\
& \otimes \sum_{j=0}^{n-i} b_{2(n-i-j)+1} \tilde{\psi}_{j} E_{(2 n-i-j+1) \delta-\alpha_{1}} \theta(n \geq 1) .
\end{aligned}
$$

Proof. We can assume that $n \geq 1$. Using Corollary 3.10 (6) and Lemma 5.3, in view of Proposition 4.7, we have

$$
\begin{aligned}
\Delta & \left(E_{2 n \delta+\alpha_{0}}\right)[4]_{1} \\
= & \Delta\left(\left[E_{n \delta-\alpha_{1}}, E_{(n+1) \delta-\alpha_{1}}\right]_{q}\right) \\
\equiv & {\left[c^{n} k_{1}^{-1} \otimes E_{n \delta-\alpha_{1}}+\left(q_{1}-q_{1}^{-1}\right) \sum_{i=1}^{n} c^{n-i} E_{i \delta-\alpha_{1}} \otimes \tilde{\psi}_{n-i},\right.} \\
& \left.c^{n+1} k_{1}^{-1} \otimes E_{(n+1) \delta-\alpha_{1}}+\left(q_{1}-q_{1}^{-1}\right) \sum_{i=1}^{n+1} c^{n-i+1} E_{i \delta-\alpha_{1}} \otimes \tilde{\psi}_{n-i+1}\right]_{q} \\
\equiv & c^{2 n+1} k_{1}^{-2} \otimes E_{2 n \delta+\alpha_{0}}[4]_{1}+\left(q_{1}-q_{1}^{-1}\right) \sum_{i=1}^{n} c^{2 n-i+1} k_{1}^{-1} E_{i \delta-\alpha_{1}} \otimes A_{i}
\end{aligned}
$$


where we set

$$
A_{i}=q^{-1} \tilde{\psi}_{n-i} E_{(n+1) \delta-\alpha_{1}}-q E_{(n+1) \delta-\alpha_{1}} \tilde{\psi}_{n-i}+\left[E_{n \delta-\alpha_{1}}, \tilde{\psi}_{n-i+1}\right] .
$$

Using Proposition 3.26 (5), we have

$$
\begin{aligned}
A_{i}= & \left(q^{-1}-q\right) \tilde{\psi}_{n-i} E_{(n+1) \delta-\alpha_{1}} \\
& -q\left(1+q^{-1}\right) \sum_{j=0}^{n-i-1} b_{2(n-i-j)} \tilde{\psi}_{j} E_{(2 n-i-j+1) \delta-\alpha_{1}} \\
& +\left(1+q^{-1}\right) \sum_{j=0}^{n-i} b_{2(n-i-j+1)} \tilde{\psi}_{j} E_{(2 n-i-j+1) \delta-\alpha_{1}} \\
= & \left(1+q^{-1}\right) \sum_{j=0}^{n-i}\left(b_{2(n-i-j+1)}-q b_{2(n-i-j)}\right) \tilde{\psi}_{j} E_{(2 n-i-j+1) \delta-\alpha_{1}} .
\end{aligned}
$$

Applying Lemma 3.14 (2), we obtain the lemma.

Corollary 5.5. Let $x \in \mathbf{U}^{+}(<)$. Then, $\Delta(x) \in \mathbf{U}^{0} \mathbf{U}^{+}(<) \otimes \mathbf{U}^{+}$.

Proof. This follows from Lemmas 5.3 and 5.4 together with Corollary 4.7 .

Lemma 5.6. Let $x \in \mathbf{U}^{+}(<)$. Then,

$$
\begin{aligned}
\Delta\left(T_{i}(x)\right) \equiv & \left(T_{\varpi_{1}} \otimes T_{\varpi_{1}}\right)(\Delta(x)) \\
& +\left(q_{1}-q_{1}^{-1}\right)\left[\left(T_{\varpi_{1}} \otimes T_{\varpi_{1}}\right)(\Delta(x)), c^{-1} k_{1} E_{\delta-\alpha_{1}} \otimes T_{\varpi_{1}}\left(E_{\alpha_{1}}\right)\right] \\
& \quad \bmod \mathbf{U}^{0} \mathbf{U}^{+}(<)_{\leq-2} \otimes \mathbf{U} .
\end{aligned}
$$

Proof. It is known [L, 37.3.2] that for $i \in I, x \in \mathbf{U}$, we have the following equality in $\sum_{\lambda, \mu \in Q} \prod_{\xi \in Q^{+}} \mathbf{U}_{\lambda+\xi} \otimes \mathbf{U}_{\mu-\xi} \subset \prod_{\lambda, \mu \in Q} \mathbf{U}_{\lambda} \otimes \mathbf{U}_{\mu}$ :

$$
\Delta\left(T_{\varpi_{1}}(x)\right)=R_{i}\left(T_{i} \otimes T_{i}\right)(\Delta(x)) R_{i}^{\prime}
$$

where we set

$$
\begin{aligned}
& R_{i}=\sum_{k \geq 0} q_{i}^{k(k-1) / 2}\left(q_{i}-q_{i}^{-1}\right)^{k}[k]_{i} ! T_{i}\left(f_{i}^{(k)}\right) \otimes T_{i}\left(e_{i}^{(k)}\right) \\
& R_{i}^{\prime}=\sum_{k \geq 0}(-1)^{k} q_{i}^{-k(k-1) / 2}\left(q_{i}-q_{i}^{-1}\right)^{k}[k]_{i} ! T_{i}\left(f_{i}^{(k)}\right) \otimes T_{i}\left(e_{i}^{(k)}\right)
\end{aligned}
$$


Noting that $T_{i}\left(f_{i}\right)=-k_{i}^{-1} e_{i}$ and $T_{i}\left(e_{i}\right)=-f_{i} k_{i}$, we have

$$
\begin{aligned}
\Delta\left(T_{\varpi_{1}}(x)\right) & =\Delta\left(T_{0}\left(T_{1}(x)\right)\right) \\
& =R_{0}\left(T_{0} \otimes T_{0}\right)\left(\Delta\left(T_{1}(x)\right)\right) R_{0}^{\prime} \\
& =R_{0}\left(T_{0} \otimes T_{0}\right)\left(R_{1}\left(T_{1} \otimes T_{1}\right)(\Delta(x)) R_{1}^{\prime}\right) R_{0}^{\prime} .
\end{aligned}
$$

Noting that $T_{\varpi_{1}}\left(f_{1}\right)=-c^{-1} k_{1} E_{\delta-\alpha_{1}}$ and $T_{\varpi_{1}}\left(e_{1}\right)=-c^{-1} \Omega\left(E_{\delta-\alpha_{1}}\right) k_{1}^{-1}$, we have

$$
\Delta\left(T_{\varpi_{1}}(x)\right)=R_{0} R_{\varpi_{1}}\left(T_{\varpi_{1}} \otimes T_{\varpi_{1}}\right)(\Delta(x)) R_{\varpi_{1}}^{\prime} R_{0}^{\prime}
$$

where we set

$$
\begin{aligned}
& R_{\varpi_{1}}=\sum_{k \geq 0} q_{1}^{k(k-1) / 2}\left(q_{1}-q_{1}^{-1}\right)^{k}[k]_{1} ! T_{\varpi_{1}}\left(f_{1}^{(k)}\right) \otimes T_{\varpi_{1}}\left(e_{1}^{(k)}\right), \\
& R_{\varpi_{1}}^{\prime}=\sum_{k \geq 0}(-1)^{k} q_{1}^{-k(k-1) / 2}\left(q_{1}-q_{1}^{-1}\right)^{k}[k]_{1} ! T_{\varpi_{1}}\left(f_{1}^{(k)}\right) \otimes T_{\varpi_{1}}\left(e_{1}^{(k)}\right) .
\end{aligned}
$$

Hence, for $x \in \mathbf{U}^{+}(<)$, in view of Corollaries 5.5 and 4.7, we have

$$
\begin{aligned}
\Delta\left(T_{\varpi_{1}}(x)\right) \equiv & \left(1-\left(q_{1}-q_{1}^{-1}\right) c^{-1} k_{1} E_{\delta-\alpha_{1}} \otimes T_{\varpi_{1}}\left(E_{\alpha_{1}}\right)\right)\left(T_{\varpi_{1}} \otimes T_{\varpi_{1}}\right)(\Delta(x)) \\
& \times\left(1+\left(q_{1}-q_{1}^{-1}\right) c^{-1} k_{1} E_{\delta-\alpha_{1}} \otimes T_{\varpi_{1}}\left(E_{\alpha_{1}}\right)\right) \\
\equiv & \left(T_{\varpi_{1}} \otimes T_{\varpi_{1}}\right)(\Delta(x)) \\
& +\left(q_{1}-q_{1}^{-1}\right)\left[\left(T_{\varpi_{1}} \otimes T_{\varpi_{1}}\right)(\Delta(x)), c^{-1} k_{1} E_{\delta-\alpha_{1}} \otimes T_{\varpi_{1}}\left(E_{\alpha_{1}}\right)\right]
\end{aligned}
$$

where $\equiv$ means the congruence modulo $\sum_{\lambda, \mu \in Q} \prod_{\xi \in Q^{+}}\left(\mathbf{U}^{0} \mathbf{U}^{+}(<)_{\leq-2} \cap \mathbf{U}_{\lambda+\xi}\right)$ $\otimes \mathbf{U}_{\mu-\xi}$. Since both sides belong to $\mathbf{U} \otimes \mathbf{U}$, we obtain the lemma.

Lemma 5.7. Let $m \geq 1, s \geq 0, t \geq 0$. Then we have

$$
\begin{aligned}
\Delta & \left(E_{2 m \delta+\alpha_{0}}^{(s)} E_{m \delta-\alpha_{1}}^{(t)}\right) \\
\equiv & c^{(2 m+1) s+m t} k_{1}^{-2 s-t} \otimes E_{2 m \delta+\alpha_{0}}^{(s)} E_{m \delta-\alpha_{1}}^{(t)} \\
& +c^{(2 m+1) s+m t-m} k_{1}^{-2 s-t+1} E_{m \delta-\alpha_{1}} \\
& \otimes\left(\left(q_{1}-q_{1}^{-1}\right) q^{-t} E_{(m+1) \delta-\alpha_{1}} E_{2 m \delta+\alpha_{0}}^{(s-1)} E_{m \delta-\alpha_{1}}^{(t)}+q_{1}^{-t+1} E_{2 m \delta+\alpha_{0}}^{(s)} E_{m \delta-\alpha_{1}}^{(t-1)}\right) \\
& +\sum_{i=1}^{m-1} c^{(2 m+1) s+m t-i} k_{1}^{-2 s-t+1} E_{i \delta-\alpha_{1}} \otimes x_{i} \theta(m \geq 2)
\end{aligned}
$$

for some $x_{i} \in \mathbf{U}^{+}$. 
Proof. Using Lemmas 5.3 and 5.4, in view of Proposition 4.7, we have

$$
\begin{aligned}
& \Delta\left(E_{2 m \delta+\alpha_{0}}^{s} E_{m \delta-\alpha_{1}}^{t}\right) \\
& \equiv\left(c^{2 m+1} k_{1}^{-2} \otimes E_{2 m \delta+\alpha_{0}}+\left(q_{1}-q_{1}^{-1}\right) c^{m+1} k_{1}^{-1} E_{m \delta-\alpha_{1}} \otimes E_{(m+1) \delta-\alpha_{1}}\right. \\
& \left.\quad+\sum_{i=1}^{m-1} c^{2 m-i+1} k_{1}^{-1} E_{i \delta-\alpha_{1}} \otimes y_{i} \theta(m \geq 2)\right)^{s} \\
& \quad \times\left(c^{m} k_{1}^{-1} \otimes E_{m \delta-\alpha_{1}}+E_{m \delta-\alpha_{1}} \otimes 1+\sum_{i=1}^{m-1} c^{m-i} E_{i \delta-\alpha_{1}} \otimes y_{i}^{\prime} \theta(m \geq 2)\right)^{t}
\end{aligned}
$$

for some $y_{i}, y_{i}^{\prime} \in \mathbf{U}^{+}$. By Corollary 3.10 (3) and Proposition 4.17, we see that

$$
\begin{aligned}
\Delta\left(E_{2 m \delta+\alpha_{0}}^{s} E_{m \delta-\alpha_{1}}^{t}\right) \equiv & c^{(2 m+1) s+m t} k_{1}^{-2 s-t} \otimes E_{2 m \delta+\alpha_{0}}^{s} E_{m \delta-\alpha_{1}}^{t} \\
& +c^{(2 m+1) s+m t-m} k_{1}^{-2 s-t+1} E_{m \delta-\alpha_{1}} \\
& \otimes\left(\left(q_{1}-q_{1}^{-1}\right)\left(q^{2(s-1)}+q^{2(s-2)} q^{-2}+\cdots+q^{-2(s-1)}\right) q^{-t}\right. \\
& \times E_{(m+1) \delta-\alpha_{1}} E_{2 m \delta+\alpha_{0}}^{s-1} E_{m \delta-\alpha_{1}}^{t} \\
& \left.+\left(1+q^{-1}+\cdots+q^{-(t-1)}\right) E_{2 m \delta+\alpha_{0}}^{s} E_{m \delta-\alpha_{1}}^{t-1}\right) \\
& +\sum_{i=1}^{m-1} c^{(2 m+1) s+m t-i} k_{1}^{-2 s-t+1} E_{i \delta-\alpha_{1}} \otimes y_{i}^{\prime \prime}
\end{aligned}
$$

for some $y_{i}^{\prime \prime} \in \mathbf{U}^{+}$. Thus, the lemma follows.

Corollary 5.8. $\quad$ Let $m \geq 1, n \delta+r \alpha \in Q^{+}$and let $x \in \mathbf{U}^{+}(<; 2 m \delta+$ $\left.\alpha_{0}, \delta-\alpha_{1}\right) \cap \mathbf{U}_{n \delta+r \alpha}^{+}$. Then we have

$$
\Delta(x) \equiv c^{n} k_{1}^{r} \otimes x+\sum_{i=1}^{m} c^{n-i} k_{1}^{r+1} E_{i \delta-\alpha_{1}} \otimes z_{i}
$$

for some $z_{i} \in \mathbf{U}^{0} \mathbf{U}^{+}\left(<; \delta-\alpha_{1}\right)$.

Proof. This follows from Lemma 5.7 together with Proposition 4.7 by the induction on $m$.

Lemma 5.9. Let $m \geq 1, n \delta+r \alpha_{1} \in Q^{+} \backslash\{0\}$ and let $x \in \mathbf{U}^{+}(<; 2 m \delta+$ $\left.\alpha_{0}, \delta-\alpha_{1}\right) \cap \mathbf{U}_{n \delta+r \alpha_{1}}^{+}$. If $\Delta(x) \equiv c^{n} k_{1}^{r} \otimes x$, then $x=0$. 
Proof. We argue by the induction on $m$. First, we consider the case where $m=1$. Then, $x=c_{s, t} E_{2 \delta+\alpha_{0}}^{(s)} E_{\delta-\alpha_{1}}^{(t)}$ for some $c_{s, t} \in \mathbf{Q}\left(q_{1}\right)$ where $s=n+r, t=$ $-2 n-3 r, s, t \geq 0$, and $s \geq 1$ or $t \geq 1$. By Lemma 5.7 with $m=1$, we have

$$
\begin{aligned}
\Delta(x) \equiv & c^{n} k_{1}^{r} \otimes x+c^{n-1} k_{1}^{r+1} E_{\delta-\alpha_{1}} \\
& \otimes c_{s, t}\left(\left(q_{1}-q_{1}^{-1}\right) q^{-t} E_{2 \delta-\alpha_{1}} E_{2 \delta+\alpha_{0}}^{(s-1)} E_{\delta-\alpha_{1}}^{(t)}+q_{1}^{-t+1} E_{2 \delta+\alpha_{0}}^{(s)} E_{\delta-\alpha_{1}}^{(t-1)}\right) .
\end{aligned}
$$

Since $s \geq 1$ or $t \geq 1$, we have $c_{s, t}=0$; thus, $x=0$. Now, assume that $m \geq 2$. Then, $x=\sum_{s, t \geq 0} c_{s, t} E_{2 m \delta+\alpha_{0}}^{(s)} E_{m \delta-\alpha_{1}}^{(t)} x_{s, t}$ where $c_{s, t} \in \mathbf{Q}\left(q_{1}\right), x_{s, t} \in \mathbf{U}^{+, h}(<$; $\left.2(m-1) \delta+\alpha_{0}, \delta-\alpha_{1}\right)$. By Lemma 5.7 and by Corollary 5.8 for $m-1$ with $x=x_{s, t}$, in view of Proposition 4.7, we have

$$
\begin{aligned}
\Delta(x) \equiv & c^{n} k_{1}^{r} \otimes x+\sum_{i=1}^{m-1} c^{n-i} k_{1}^{r+1} E_{i \delta-\alpha_{1}} \otimes w_{i} \\
& +c^{n-m} k_{1}^{r+1} E_{m \delta-\alpha_{1}} \\
& \otimes \sum_{s, t \geq 0} c_{s, t}\left(\left(q_{1}-q_{1}^{-1}\right) q^{-t} E_{(m+1) \delta-\alpha_{1}} E_{2 m \delta+\alpha_{0}}^{(s-1)} E_{m \delta-\alpha_{1}}^{(t)}\right. \\
& \left.+q_{1}^{-t+1} E_{2 m \delta+\alpha_{0}}^{(s)} E_{m \delta-\alpha_{1}}^{(t-1)}\right) x_{s, t}
\end{aligned}
$$

for some $w_{i} \in \mathbf{U}^{+}$. Since $\Delta(x) \equiv c^{n} k_{1} \otimes x$, we have $\left(w_{i}=0\right.$ for $1 \leq i \leq m-1$, and) $c_{s, t} x_{s, t}=0$ unless $s=t=0$. Thus, $x \in \mathbf{U}^{+, h}\left(<; 2(m-1) \delta+\alpha_{0}, \delta-\alpha_{1}\right)$. Applying the induction hypothesis, we obtain $x=0$. Thus, the induction proceeds.

Corollary 5.10. Let $n \delta+r \alpha_{1} \in Q^{+} \backslash\{0\}$ and let $x \in \mathbf{U}^{+}\left(<; \delta-\alpha_{1}\right) \cap$ $\mathbf{U}_{n \delta+r \alpha_{1}}^{+}$. If $\Delta(x) \equiv c^{n} k_{1}^{r} \otimes x$, then $x=0$.

Proof. This follows from Lemma 5.9.

\section{$\S 6 . \quad$ Inner Product}

We set $\mathbf{A}=\mathbf{Q}\left(q_{1}\right) \cap \mathbf{Z}\left[\left[q_{1}^{-1}\right]\right] \subset \mathbf{Q}\left(\left(q_{1}^{-1}\right)\right)$.

Definition 6.1 [L, 1.2.2, 3.1.5]. Let $r$ be the $\mathbf{Q}\left(q_{1}\right)$-linear map from $\mathbf{U}^{+}$to $\mathbf{U}^{+} \otimes \mathbf{U}^{+}$defined as the composition of $\Delta: \mathbf{U}^{+} \rightarrow \oplus_{\xi, \mu \in Q^{+}}\left(\mathbf{U}_{\xi}^{+} k_{\mu} \otimes \mathbf{U}_{\mu}^{+}\right)$ and the vector space isomorphism from $\oplus_{\xi, \mu \in Q^{+}}\left(\mathbf{U}_{\xi}^{+} k_{\mu} \otimes \mathbf{U}_{\mu}^{+}\right)$to $\oplus_{\xi, \mu \in Q^{+}}\left(\mathbf{U}_{\xi}^{+} \otimes\right.$ $\left.\mathbf{U}_{\mu}^{+}\right)=\mathbf{U}^{+} \otimes \mathbf{U}^{+}$that sends $x k_{\mu} \otimes x^{\prime}$ to $x \otimes x^{\prime}$ for $x \in \mathbf{U}_{\xi}^{+}$and $x^{\prime} \in \mathbf{U}_{\mu}^{+}$. 
Lemma 6.2. Let $n \geq 1$. Then,

$$
r\left(E_{n \delta-\alpha_{1}}\right) \equiv 1 \otimes E_{n \delta-\alpha_{1}}+\left(q_{1}-q_{1}^{-1}\right) \sum_{i=1}^{n} E_{i \delta-\alpha_{1}} \otimes \tilde{\psi}_{n-i}
$$

where $\equiv$ means the congruence modulo $\mathbf{U}^{+}(<) \leq-2 \otimes \mathbf{U}^{+}$.

Proof. This follows from Lemma 5.3.

Let us recall the inner product on $\mathbf{U}^{+}$introduced by Drinfeld.

Definition 6.3 [K1, 3.4.4, 3.4.8], [L, 1.2.3, 1.2.5, 1.2.13]. Let $(\cdot, \cdot)$ be the $\mathbf{Q}\left(q_{1}\right)$-valued symmetric bilinear form on $\mathbf{U}^{+}$such that

(1) $(1,1)=1$,

(2) $\left(E_{i}, E_{j}\right)=\delta_{i j}\left(1-q_{i}^{-2}\right)^{-1}$ for $i, j \in I$,

(3) $\left(x, y y^{\prime}\right)=\left(r(x), y \otimes y^{\prime}\right)$ for $x, y, y^{\prime} \in \mathbf{U}^{+}$,

(4) $\left(E_{i} x, y\right)=\left(E_{i}, E_{i}\right)\left(x,{ }_{i} r(y)\right)$ for $x, y \in \mathbf{U}^{+}, i \in I$,

(5) $\left(x E_{i}, y\right)=\left(E_{i}, E_{i}\right)\left(x, r_{i}(y)\right)$ for $x, y \in \mathbf{U}^{+}, i \in I$,

(6) for $\xi, \mu \in Q^{+}$such that $\xi \neq \mu$ and for $x \in \mathbf{U}_{\xi}^{+}, y \in \mathbf{U}_{\mu}^{+}$, we have $(x, y)=0$.

(7) for $\nu \in Q^{+}$, the restriction of $(\cdot, \cdot)$ on $\mathbf{U}_{\nu}^{+}$is non-degenerate,

In $(3)$, the bilinear form on $\mathbf{U}^{+} \otimes \mathbf{U}^{+}$that sends a pair $\left(x_{1} \otimes x_{2}, x_{1}^{\prime} \otimes x_{2}^{\prime}\right)$ to $\left(x_{1}, x_{2}\right)\left(x_{1}^{\prime}, x_{2}^{\prime}\right) \in \mathbf{Q}\left(q_{1}\right)$ is also denoted by $(\cdot, \cdot)$.

Proposition 6.4 [L, 40.2.4]. Let $\mathbf{c}_{+}=\left(\mathbf{c}_{+_{i}}\right), \quad \mathbf{c}_{+}^{\prime}=\left(\mathbf{c}_{+i}^{\prime}\right) \in$ $\oplus_{i \in R_{r e}^{+}(>)} \mathbf{Z}_{\geq 0}^{i}$, let $\mathbf{c}_{-}=\left(\mathbf{c}_{-i}\right), \mathbf{c}_{-}^{\prime}=\left(\mathbf{c}_{-i}^{\prime}\right) \in \oplus_{i \in R_{r e}^{+}(>)} \mathbf{Z}_{\geq 0}^{i}$, and let $x, x^{\prime} \in$ $\mathbf{U}^{+}(0)$. Then,

(1) $\left(\mathbf{E}_{\mathbf{c}_{+}} x \mathbf{E}_{\mathbf{c}_{-}}, \mathbf{E}_{\mathbf{c}_{+}^{\prime}} x^{\prime} \mathbf{E}_{\mathbf{c}_{-}^{\prime}}\right)=\delta_{\mathbf{c}_{+}, \mathbf{c}_{+}^{\prime}} \delta_{\mathbf{c}_{-}, \mathbf{c}_{-}^{\prime}}\left(\mathbf{E}_{\mathbf{c}_{+}}, \mathbf{E}_{\mathbf{c}_{+}}\right)\left(x, x^{\prime}\right)\left(\mathbf{E}_{\mathbf{c}_{-}}, \mathbf{E}_{\mathbf{c}_{-}}\right)$,

(2) $\left(\mathbf{E}_{\mathbf{c}_{+}}, \mathbf{E}_{\mathbf{c}_{+}}\right) \equiv 1 \bmod q_{1}^{-1} \mathbf{A}$,

(3) $\left(\mathbf{E}_{\mathbf{c}_{-}}, \mathbf{E}_{\mathbf{c}_{-}}\right) \equiv 1 \bmod q_{1}^{-1} \mathbf{A}$.

Proof. If we set $\mathbf{h}=\left(i_{k}\right)_{k \in \mathbf{Z}}$ where $i_{k}$ is 0 if $k$ is even and 1 otherwise, and $p=0$, then our symbols $\mathbf{U}^{+}(>)$and $\mathbf{U}^{+}(<)$coincide with those in $[\mathrm{L}$, 40.2.5], and $\mathbf{U}^{+}(0)$ is contained in the space $\mathbf{P}$ defined in [L, 40.2.3] by Lemma 4.10 and the fact that $\mathbf{U}^{+}$is invariant under $*$.

Remark 6.5. It follows from Corollary 4.20 that $\mathbf{U}^{+}(0)$ coincides with the above $\mathbf{P}$. 
Proposition 6.6. The $\mathbf{Q}\left(q_{1}\right)$-linear map $\mathbf{U}^{+}(>) \otimes \mathbf{U}^{+}(0) \otimes \mathbf{U}^{+}(<) \longrightarrow$ $\mathbf{U}^{+}$given by multiplication is an isomorphism.

Proof. By Corollary 4.20, it is enough to show the injectivity. Let $\sum \mathbf{E}_{\mathbf{c}_{+}} x_{\mathbf{c}_{+}, \mathbf{c}_{-}} \mathbf{E}_{\mathbf{c}_{-}}=0$ where the sum is taken over $\mathbf{c}_{+} \in \oplus_{i \in R_{r e}^{+}(>)} \mathbf{Z}_{\geq 0}^{i}$ and $\mathbf{c}_{-} \in \oplus_{i \in R_{r e}^{+}(<)} \mathbf{Z}_{\geq 0}^{i}$; and $x_{\mathbf{c}_{+}, \mathbf{c}_{-}} \in \mathbf{U}^{+}(0)$. We fix $\mathbf{c}_{+} \in \oplus_{i \in R_{r e}^{+}(>)} \mathbf{Z}_{\geq 0}^{i}$ and $\mathbf{c}_{-} \in \oplus_{i \in R_{r e}^{+}(<)} \mathbf{Z}_{\geq 0}^{i}$. Then, for any $x \in \mathbf{U}^{+}(0)$, by Proposition 6.4, we have $\left(\mathbf{E}_{\mathbf{c}_{+}}, \mathbf{E}_{\mathbf{c}_{+}}\right)\left(x_{\mathbf{c}_{+}, \mathbf{c}_{-}}, x\right)\left(\mathbf{E}_{\mathbf{c}_{-}}, \mathbf{E}_{\mathbf{c}_{-}}\right)=0$; thus, $\left(x_{\mathbf{c}_{+}, \mathbf{c}_{-}}, x\right)=0$. Hence, by Proposition 6.4 together with Corollary 4.20 , we have $\left(x_{\mathbf{c}_{+}, \mathbf{c}_{-}}, x^{\prime}\right)=0$ for any $x^{\prime} \in \mathbf{U}^{+}$. In view of Definition $6.3(7)$, we conclude that $x_{\mathbf{c}_{+}, \mathbf{c}_{-}}=0$. The proposition is proved.

Lemma 6.7. Let $n \geq 1$. Then,

$$
\left(\tilde{\psi}_{n}, \tilde{\psi}_{n}\right)=\left(1+q^{-1}\right)\left(q_{1}-q_{1}^{-1}\right)^{-2} b_{2 n}
$$

Proof. By Definitions 3.3 and $6.3((4),(5))$, we have

$$
\begin{aligned}
\left(\tilde{\psi}_{n}, \tilde{\psi}_{n}\right) & =\left(E_{n \delta-\alpha_{1}} E_{\alpha_{1}}-q^{-1} E_{\alpha_{1}} E_{n \delta-\alpha_{1}}, \tilde{\psi}_{n}\right) \\
& =\left(E_{\alpha_{1}}, E_{\alpha_{1}}\right)\left(E_{n \delta-\alpha_{1}}, r_{1}\left(\tilde{\psi}_{n}\right)-{ }_{1} r\left(\tilde{\psi}_{n}\right) q^{-1}\right) .
\end{aligned}
$$

Applying Corollary 3.28, we have

$$
\left(\tilde{\psi}_{n}, \tilde{\psi}_{n}\right)=\left(E_{\alpha_{1}}, E_{\alpha_{1}}\right)\left(q_{1}-q_{1}^{-1}\right) q^{-1}\left(1+q^{-1}\right) \sum_{i=1}^{n} b_{2 i}\left(E_{n \delta-\alpha_{1}}, \tilde{\psi}_{n-i} E_{i \delta-\alpha_{1}}\right) .
$$

By Definition $6.3((3),(6))$ and Lemma 6.2, we have

$$
\left(\tilde{\psi}_{n}, \tilde{\psi}_{n}\right)=\left(E_{\alpha_{1}}, E_{\alpha_{1}}\right)\left(E_{n \delta-\alpha_{1}}, E_{n \delta-\alpha_{1}}\right) q^{-1}\left(1+q^{-1}\right) b_{2 n} .
$$

Since $\left(E_{n \delta-\alpha_{1}}, E_{n \delta-\alpha_{1}}\right)=\left(E_{\alpha_{1}}, E_{\alpha_{1}}\right)=\left(1-q^{-1}\right)^{-1}$ by [L, 40.2.4], we obtain the desired result.

Lemma 6.8. Let $n, r \geq 1$, let $\beta \in R_{r e}^{+}(<)$, and let $x \in \mathbf{U}^{+}(<; \beta) \cap$ $\mathbf{U}_{n \delta-r \alpha_{1}}^{+}$. Then, $\left[x, E_{\alpha_{1}}\right]_{q^{-r}} \in \mathbf{U}^{+}(0) \mathbf{U}^{+}(<; \beta)$.

Proof. We argue by the induction on $r$. First, assume that $x=x_{1} x_{2}$ for some $x_{i} \in \mathbf{U}^{+}(0) \cap \mathbf{U}_{n_{i} \delta-r_{i} \alpha_{1}}^{+}$with $r_{i} \geq 1$. By Lemma 2.11, we have

$$
\begin{aligned}
{\left[x, E_{\alpha_{1}}\right]_{q^{-r}} } & =\left[x_{1} x_{2}, E_{\alpha_{1}}\right]_{q^{-r_{1}-r_{2}}} \\
& =x_{1}\left[x_{2}, E_{\alpha_{1}}\right]_{q^{-r_{2}}}+\left[x_{1}, E_{\alpha_{1}}\right]_{q^{-r_{1}}} x_{2} q^{-r_{2}},
\end{aligned}
$$


and this belongs to $\mathbf{U}^{+}(0) \mathbf{U}^{+}(<; \beta)$ by the induction hypothesis and Proposition 4.17. Thus, the lemma is reduced to the case where $x=E_{n \delta-\alpha_{1}}$ or $x=E_{2 m \delta-\alpha_{0}}$ with $m \geq 1$, but it follows from Proposition 3.26 (2) and Corollary $3.27(1)$.

Lemma 6.9. Let $n \geq 0$. Then,

(1) $\Delta\left(\tilde{\psi}_{n}\right) \equiv\left(q_{1}-q_{1}^{-1}\right) \sum_{i=0}^{n} c^{n-i} \tilde{\psi}_{i} \otimes \tilde{\psi}_{n-i}$,

(2) $\Delta\left(P_{n}\right) \equiv \sum_{i=0}^{n} c^{n-i} P_{i} \otimes P_{n-i}$

where $\equiv$ means the congruence modulo $\mathbf{U}^{0} \mathbf{U}^{+}(0) \mathbf{U}^{+}(<)_{\leq-1} \otimes \mathbf{U}^{+}$.

Proof. (1) We can assume that $n \geq 1$. Note that in Lemma 6.2, the truncated part can be written as $\sum_{\xi, \mu \in Q^{+} ; \xi+\mu=n \delta-\alpha_{1}} k_{\mu} x_{\xi} \otimes y_{\mu}$ with $x_{\xi} \in$ $\mathbf{U}^{+}(<)_{\leq-2} \cap \mathbf{U}_{\xi}^{+}, y_{\mu} \in \mathbf{U}_{\mu}^{+}$, for which we have $\left[k_{\mu} x_{\xi}, E_{\alpha_{1}}\right]_{q^{-1}}=k_{\mu}\left[x_{\xi}, E_{\alpha_{1}}\right]_{q^{\mathrm{h}(\xi)}}$ and this belongs to $\mathbf{U}^{0} \mathbf{U}^{+}(0) \mathbf{U}^{+}(<) \leq-1$ by Lemma 6.8. Hence, using Definition 3.3 and Lemma 6.2, we have

$$
\begin{aligned}
\Delta\left(\tilde{\psi}_{n}\right)= & {\left[\Delta\left(E_{n \delta-\alpha_{1}}\right), \Delta\left(E_{\alpha_{1}}\right)\right]_{q^{-1}} } \\
\equiv & {\left[c^{n} k_{1}^{-1} \otimes E_{n \delta-\alpha_{1}}+\left(q_{1}-q_{1}^{-1}\right) \sum_{i=1}^{n} c^{n-i} E_{i \delta-\alpha_{1}} \otimes \tilde{\psi}_{n-i}\right.} \\
& \left.k_{1} \otimes E_{\alpha_{1}}+E_{\alpha_{1}} \otimes 1\right]_{q^{-1}} \\
\equiv & c^{n} \otimes \tilde{\psi}_{n}+c^{n}\left(k_{1}^{-1} E_{\alpha_{1}}-q^{-1} E_{\alpha_{1}} k_{1}^{-1}\right) \otimes E_{n \delta-\alpha_{1}} \\
& +\left(q_{1}-q_{1}^{-1}\right) \sum_{i=1}^{n} c^{n-i} \tilde{\psi}_{i} \otimes \tilde{\psi}_{n-i} .
\end{aligned}
$$

The second term vanishes and we obtain (1).

(2) We argue by the induction on $n$. We can assume that $n \geq 1$. By Definition 4.9, the induction hypothesis, (1), and Proposition 4.17, we have

$$
\begin{aligned}
\Delta\left(P_{n}\right) & {[2 n]_{1} } \\
& =\sum_{k=0}^{n-1} \Delta\left(P_{k} \tilde{\psi}_{n-k}\right) q^{-k} \\
& \equiv \sum_{k=0}^{n-1}\left(\sum_{i=0}^{k} c^{k-i} P_{i} \otimes P_{k-i}\right)\left(\sum_{j=0}^{n-k} c^{n-k-j} \tilde{\psi}_{j} \otimes \tilde{\psi}_{n-k-j}\right) q^{-k}\left(q_{1}-q_{1}^{-1}\right) \\
& =\sum_{k=0}^{n-1} \sum_{i=0}^{k} \sum_{j=0}^{n-k} c^{n-i-j} P_{i} \tilde{\psi}_{j} \otimes P_{k-i} \tilde{\psi}_{n-k-j} q^{-k}\left(q_{1}-q_{1}^{-1}\right) .
\end{aligned}
$$


Putting $i+j=i^{\prime}, i=j^{\prime}, k-i=k^{\prime}$, we see that $j=i^{\prime}-j^{\prime}, k=j^{\prime}+k^{\prime}$ and that $0 \leq k \leq n-1,0 \leq i \leq k, 0 \leq j \leq n-k$ is equivalent to $0 \leq i^{\prime} \leq n, 0 \leq$ $j^{\prime} \leq i^{\prime}, 0 \leq k^{\prime} \leq \min \left(n-i^{\prime}, n-j^{\prime}-1\right)$. Hence,

$$
\begin{aligned}
& \Delta\left(P_{n}\right)[2 n]_{1} \\
& \equiv \sum_{i=0}^{n} \sum_{j=0}^{i} \sum_{k=0}^{\min (n-i, n-j-1)} c^{n-i} P_{j} \tilde{\psi}_{i-j} \otimes P_{k} \tilde{\psi}_{n-i-k} q^{-j-k}\left(q_{1}-q_{1}^{-1}\right) \\
& =\sum_{i=0}^{n}\left(\sum_{k=0}^{n-i-1} c^{n-i} P_{i} \tilde{\psi}_{0} \otimes P_{k} \tilde{\psi}_{n-i-k} q^{-i-k}\right. \\
& \quad+\theta(i \geq 1) \sum_{j=0}^{i-1} c^{n-i} P_{j} \tilde{\psi}_{i-j} q^{-j} \\
& \left.\quad \otimes\left(P_{n-i} \tilde{\psi}_{0} q^{-n+i}+P_{n-i}[2 n-2 i]_{1}\right)\right)\left(q_{1}-q_{1}^{-1}\right) \\
& =\sum_{i=0}^{n} c^{n-i} P_{i} \otimes P_{n-i}\left([2 n-2 i]_{1} q^{-i}+[2 i]_{1}\left(q^{-n+i}+[2 n-2 i]_{1}\left(q_{1}-q_{1}^{-1}\right)\right)\right) .
\end{aligned}
$$

Thus, the induction proceeds.

Corollary 6.10. Let $n \geq 0$. Then,

(1) $r\left(\tilde{\psi}_{n}\right) \equiv \sum_{i=0}^{n} \tilde{\psi}_{i} \otimes \tilde{\psi}_{n-i} \bmod \mathbf{U}^{+}(0) \mathbf{U}^{+}(<)_{\leq-1} \otimes \mathbf{U}^{+}$,

(2) $r\left(P_{n}\right) \equiv \sum_{i=0}^{n} P_{i} \otimes P_{n-i} \bmod \mathbf{U}^{+}(0) \mathbf{U}^{+}(<)_{\leq-1} \otimes \mathbf{U}^{+}$.

Proof. This follows from Lemma 6.9.

Lemma 6.11. For $n \geq 0$, we have

$$
\left(\tilde{\psi}_{n}, P_{n}\right)=\left(q_{1}-q_{1}^{-1}\right)^{-1}[2 n+1]_{1} .
$$

Proof. We argue by the induction on $n$. We can assume that $n \geq 1$. Using Corollary 6.10 (1) and Definition 4.9, in view of Definition 6.3 (6), we have

$$
\begin{aligned}
\left(\tilde{\psi}_{n}, P_{n}\right)[2 n]_{1} & =\left(\sum_{i=0}^{n} \tilde{\psi}_{i} \otimes \tilde{\psi}_{n-i}, \sum_{j=0}^{n-1} P_{j} \otimes \tilde{\psi}_{n-j} q^{-j}\right)\left(q_{1}-q_{1}^{-1}\right) \\
& =\sum_{i=0}^{n-1}\left(\tilde{\psi}_{i}, P_{i}\right)\left(\tilde{\psi}_{n-i}, \tilde{\psi}_{n-i}\right) q^{-i}\left(q_{1}-q_{1}^{-1}\right) .
\end{aligned}
$$


Applying the induction hypothesis and Lemma 6.7, we have

$$
\left(\tilde{\psi}_{n}, P_{n}\right)[2 n]_{1}=\sum_{i=0}^{n-1} b_{2(n-i)}[2 i+1]_{1} q^{-i}\left(1+q^{-1}\right)\left(q_{1}-q_{1}^{-1}\right)^{-2} .
$$

By Lemma 3.17 (1), the induction proceeds.

Lemma 6.12. Let $n \geq 0$. Then,

$$
\left(P_{n}, P_{n}\right) \equiv 1 \quad \bmod q_{1}^{-1} \mathbf{A} .
$$

Proof. We argue by the induction on $n$. We can assume that $n \geq 1$. By Corollary 6.10 (2) and Definition 4.9, and in view of Definition 6.3 (6), we have

$$
\begin{aligned}
\left(P_{n}, P_{n}\right)[2 n]_{1} & =\left(\sum_{i=0}^{n} P_{i} \otimes P_{n-i}, \sum_{j=0}^{n-1} P_{j} \otimes \tilde{\psi}_{n-j} q^{-j}\right) \\
& =\sum_{i=1}^{n}\left(P_{n-i}, P_{n-i}\right)\left(P_{i}, \tilde{\psi}_{i}\right) q^{-n+i} .
\end{aligned}
$$

Applying the induction hypothesis and Lemma 6.11, we have

$$
\begin{aligned}
\left(P_{n}, P_{n}\right) & =\sum_{i=1}^{n}\left(1+\alpha_{i}\right)[2 n]_{1}^{-1}\left(q_{1}-q_{1}^{-1}\right)^{-1}[2 i+1]_{1} q^{-n+i} \\
& =\sum_{i=1}^{n}\left(1+\alpha_{i}\right)\left(q^{n}-q^{-n}\right)^{-1}\left(q^{i}+q^{i-1}+\cdots+q^{-i}\right) q^{-n+i} \\
& =\sum_{i=1}^{n}\left(1+\alpha_{i}\right)\left(1+q^{-1}+\cdots+q^{-2 i}\right)\left(1-q^{-2 n}\right)^{-1} q^{-2 n+2 i}
\end{aligned}
$$

for some $\alpha_{i} \in q_{1}^{-1} \mathbf{A}$. Thus, $\left(P_{n}, P_{n}\right) \equiv 1 \bmod q_{1}^{-1} \mathbf{A}$ and the induction proceeds.

Definition 6.13 (see [M, Chapter 1]). Let us recall the Z-algebra $\Lambda$ of symmetric functions.

(1) For $n \geq 1$, let $h_{n} \in \Lambda$ be the complete symmetric functions, which are algebraically independent and generate $\Lambda$. We also set $h_{0}=1$.

(2) We define the grading on $\Lambda$ by $\operatorname{deg}\left(h_{n}\right)=n$ for $n \geq 0$.

(3) Let $\Delta^{\prime}$ be the algebra homomorphism from $\Lambda$ to $\Lambda \otimes \Lambda$ given by $\Delta^{\prime}\left(h_{n}\right)=$ $\sum_{i=0}^{n} h_{i} \otimes h_{n-i}$. 
(4) Let $(\cdot, \cdot)_{\Lambda}$ be the $\mathbf{Z}$-valued positive definite symmetric bilinear form determined by the following properties: $\left(h_{n}, h_{n}\right)_{\Lambda}=1$ for $n \geq 0 ;(f, g)_{\Lambda}=0$ for homogeneous $f, g \in \Lambda$ with $\operatorname{deg}(f) \neq \operatorname{deg}(g)$; and $(f, g h)_{\Lambda}=\left(\Delta^{\prime}(f), g \otimes\right.$ $h)_{\Lambda \otimes \Lambda}$ for $f, g, h \in \Lambda$. Here, $(\cdot, \cdot)_{\Lambda \otimes \Lambda}$ is the symmetric bilinear form on $\Lambda \otimes$ $\Lambda$ such that $\left(f_{1} \otimes f_{2}, g_{1} \otimes g_{2}\right)_{\Lambda \otimes \Lambda}=\left(f_{1}, g_{1}\right)_{\Lambda}\left(f_{2}, g_{2}\right)_{\Lambda}$ for $f_{1}, f_{2}, g_{1}, g_{2} \in \Lambda$.

(5) Let $s_{\lambda} \in \Lambda$ be the Schur functions where $\lambda$ runs through the set of partitions. The $s_{\lambda}$ form an orthonormal basis of $\Lambda$ with respect to $(\cdot, \cdot)_{\Lambda}$.

Definition 6.14. Let $S$ be the $\mathbf{Z}$-subalgebra of $\mathbf{U}^{+}(0)$ generated by $P_{n}$ for $n \geq 1$. Let $\varphi$ be the surjective $\mathbf{Z}$-algebra homomorphism from $\mathbf{Z}\left[h_{1}, h_{2}, \cdots\right]$ to $S$ given by $\varphi\left(h_{n}\right)=P_{n}$ for $n \geq 1$.

Lemma 6.15. Let $f \in \Lambda$. Then,

$$
r(\varphi(f)) \equiv(\varphi \otimes \varphi)\left(\Delta^{\prime}(f)\right) \quad \bmod \mathbf{U}^{+}(0) \mathbf{U}^{+}(<) \leq-1 \otimes \mathbf{U}^{+} .
$$

Proof. Let $f, g \in \Lambda$. By Lemma 6.9 (2) and Proposition 4.17, we have

$$
\begin{aligned}
\Delta(\varphi(f)) & \equiv \sum_{i} c^{n_{i}} x_{i} \otimes y_{i} \quad \bmod \mathbf{U}^{0} \mathbf{U}^{+}(0) \mathbf{U}^{+}(<)_{\leq-1} \otimes \mathbf{U}^{+}, \\
\Delta(\varphi(g)) & \equiv \sum_{j} c^{n_{j}^{\prime}} x_{j}^{\prime} \otimes y_{j}^{\prime} \quad \bmod \mathbf{U}^{0} \mathbf{U}^{+}(0) \mathbf{U}^{+}(<)_{\leq-1} \otimes \mathbf{U}^{+}
\end{aligned}
$$

for some $x_{i}, y_{i}, x_{j}^{\prime}, y_{j}^{\prime} \in S^{h}$ with $n_{i}=\mathrm{i}\left(y_{i}\right), n_{j}^{\prime}=\mathrm{i}\left(y_{j}^{\prime}\right)$. Then,

$$
\begin{aligned}
& r(\varphi(f)) \equiv \sum_{i} x_{i} \otimes y_{i} \quad \bmod \mathbf{U}^{+}(0) \mathbf{U}^{+}(<)_{\leq-1} \otimes \mathbf{U}^{+} \\
& r(\varphi(g)) \equiv \sum_{i} x_{i}^{\prime} \otimes y_{i}^{\prime} \quad \bmod \mathbf{U}^{+}(0) \mathbf{U}^{+}(<)_{\leq-1} \otimes \mathbf{U}^{+}
\end{aligned}
$$

By Proposition 4.17, we have

$$
\begin{aligned}
\Delta(\varphi(f g)) & =\Delta(\varphi(f) \varphi(g))=\Delta(\varphi(f)) \Delta(\varphi(g)) \\
& \equiv \sum_{i, j} c^{n_{i}+n_{j}^{\prime}} x_{i} x_{j}^{\prime} \otimes y_{i} y_{j}^{\prime} \quad \bmod \mathbf{U}^{0} \mathbf{U}^{+}(0) \mathbf{U}^{+}(<)_{\leq-1} \otimes \mathbf{U}^{+}
\end{aligned}
$$

and

$$
r(\varphi(f)) r(\varphi(g)) \equiv \sum_{i, j} x_{i} x_{j}^{\prime} \otimes y_{i} y_{j}^{\prime} \quad \bmod \mathbf{U}^{+}(0) \mathbf{U}^{+}(<)_{\leq-1} \otimes \mathbf{U}^{+} .
$$

Thus,

$$
r(\varphi(f g)) \equiv r(\varphi(f)) r(\varphi(g)) \quad \bmod \mathbf{U}^{+}(0) \mathbf{U}^{+}(<)_{\leq-1} \otimes \mathbf{U}^{+}
$$


On the other hand, we have

$$
(\varphi \otimes \varphi)\left(\Delta^{\prime}(f g)\right)=(\varphi \otimes \varphi)\left(\Delta^{\prime}(f)\right)(\varphi \otimes \varphi)\left(\Delta^{\prime}(g)\right) .
$$

Thus, the lemma is reduced to the case where $f=h_{n}$ for $n \geq 0$, but it follows from Corollary $6.10(2)$ and Definition 6.3 (4).

Lemma 6.16. Let $f, g \in \Lambda$. Then,

(1) $(\varphi(f), \varphi(g)) \in \mathbf{A}$,

(2) $(\varphi(f), \varphi(g)) \equiv(f, g)_{\Lambda} \bmod q_{1}^{-1} \mathbf{A}$.

Proof. We can assume that $f, g \in \Lambda$ are homogeneous. We can also assume that $\operatorname{deg}(f)=\operatorname{deg}(g)$; otherwise, both sides vanish by Definitions 6.3 (6) and 6.13 (4). We prove (1) and (2) at once by the induction on $\operatorname{deg}(f)$. First, assume that $g=g_{1} g_{2}$ for homogeneous $g_{i} \in \Lambda$ with $\operatorname{deg}\left(g_{i}\right) \geq 1$. Then, by Lemma 6.16 and Definition 6.3 (6), we have

$$
\begin{aligned}
(\varphi(f), \varphi(g)) & =\left(\varphi(f), \varphi\left(g_{1}\right) \varphi\left(g_{2}\right)\right) \\
& =\left(r(\varphi(f)), \varphi\left(g_{1}\right) \otimes \varphi\left(g_{2}\right)\right) \\
& =\left((\varphi \otimes \varphi)\left(\Delta^{\prime}(f)\right), \varphi\left(g_{1}\right) \otimes \varphi\left(g_{2}\right)\right) .
\end{aligned}
$$

Hence, by the induction hypothesis, we obtain $(1):(\varphi(f), \varphi(g)) \in \mathbf{A}$; and

$$
(\varphi(f), \varphi(g)) \equiv\left(\Delta^{\prime}(f), g_{1} \otimes g_{2}\right)_{\Lambda \otimes \Lambda} \quad \bmod q_{1}^{-1} \mathbf{A} .
$$

Hence, in view of Definition $6.13(4)$, we obtain $(2):(\varphi(f), \varphi(g)) \equiv(f, g)_{\Lambda}$ $\bmod q_{1}^{-1} \mathbf{A}$, and the induction proceeds. Thus, the lemma is reduced to the case where $g=h_{n}$ for $n \geq 0$. Similarly, we can also assume that $f=h_{n}$ for $n \geq 0$. Thus, the lemma follows from Lemma 6.12 and Definition 6.13 (4).

Definition 6.17. For a partition $\lambda$, we set $\mathbf{S}_{\lambda}=\varphi\left(s_{\lambda}\right)$.

Proposition 6.18. We have

(1) $\varphi$ is an isomorphism of $\mathbf{Z}$-algebras from $\Lambda$ to $S$,

(2) $\left(\mathbf{S}_{\lambda}, \mathbf{S}_{\mu}\right) \equiv \delta_{\lambda, \mu} \bmod q_{1}^{-1} \mathbf{A}$,

(3) the $\mathbf{S}_{\lambda}$ form a $\mathbf{Q}\left(q_{1}\right)$-basis of $\mathbf{U}^{+}(0)$,

(4) both of $\left\{P_{n} \mid n \geq 1\right\}$ and $\left\{\tilde{\psi}_{n} \mid n \geq 1\right\}$ are algebraically independent over $\mathbf{Q}\left(q_{1}\right)$. 
Proof. By virtue of Lemma 6.16, if we define the $\mathbf{Z}$-valued symmetric bilinear form $(\cdot, \cdot)^{\prime}$ on $S$ as the composition of $(\cdot, \cdot)$ and the canonical projection from $\mathbf{A}$ to $\mathbf{A} / q_{1}^{-1} \mathbf{A}=\mathbf{Z}$, then we have

$$
(\varphi(f), \varphi(g))^{\prime}=(f, g)_{\Lambda} \quad \text { for } f, g \in \Lambda .
$$

Since $(\cdot, \cdot)_{\Lambda}$ is positive definite, we see that $\varphi$ is injective; hence, we obtain (1). Thus, the $\mathbf{S}_{\lambda}$ 's form a $\mathbf{Z}$-basis of $S$ orthonormal with respect to $(\cdot, \cdot)^{\prime}$; hence, they span $\mathbf{U}^{+}(0)$ over $\mathbf{Q}\left(q_{1}\right)$ and satisfy (2). Now, let $\sum_{\lambda} c_{\lambda} \mathbf{S}_{\lambda}=0$ with $c_{\lambda} \in \mathbf{Z}\left[q_{1}\right]$. Assume that there exists a nonzero $c_{\lambda}$. For each of such $c_{\lambda}$, take the smallest $n_{\lambda} \geq 0$ such that $c_{\lambda} q_{1}^{-n_{\lambda}} \in \mathbf{Z}+q_{1}^{-1} \mathbf{Z}\left[q_{1}^{-1}\right]$. Let $n_{\lambda_{0}}$ be the largest of $n_{\lambda}$. Then, $c_{\lambda} q_{1}^{-n_{\lambda_{0}}} \in \mathbf{A}$ and $c_{\lambda_{0}} q_{1}^{-n_{\lambda_{0}}} \notin q_{1}^{-1} \mathbf{A}$. Then, $0=\left(q_{1}^{-n_{\lambda_{0}}} \sum c_{\lambda} \mathbf{S}_{\lambda}, \mathbf{S}_{\lambda_{0}}\right) \equiv$ $c_{\lambda_{0}} q_{1}^{-n_{\lambda_{0}}} \bmod q_{1}^{-1} \mathbf{A}$. A contradiction is deduced. Hence, the $\mathbf{S}_{\lambda}$ 's are linearly independent over $\mathbf{Z}\left[q_{1}\right]$; thus, they are linearly independent over $\mathbf{Q}\left(q_{1}\right)$. (3) is proved. It follows from (3) that for $n \geq 1$, the dimension of $\mathbf{U}^{+}(0) \cap \mathbf{U}_{n \delta}^{+}$over $\mathbf{Q}\left(q_{1}\right)$ is equal to the partition number of $n$. (4) follows.

Theorem 6.19. Each of the following is a $\mathbf{Q}\left(q_{1}\right)$-basis of $\mathbf{U}^{+}$:

(1) $\left\{\mathbf{E}_{\mathbf{c}_{+}} \mathbf{E}_{\mathbf{c}_{\mathbf{0}}}^{\prime} \mathbf{E}_{\mathbf{c}_{-}} \mid \mathbf{c}_{+} \in \oplus_{i \in R_{r e}^{+}(>)} \mathbf{Z}_{\geq 0}^{(i)}, \mathbf{c}_{\boldsymbol{0}} \in \oplus_{n \geq 1} \mathbf{Z}_{\geq 0}^{(n)}, \mathbf{c}_{-} \in \oplus_{i \in R_{r e}^{+}(<)} \mathbf{Z}_{\geq 0}^{(i)}\right\}$,

(2) $\left\{\mathbf{E}_{\mathbf{c}_{+}} \mathbf{E}_{\mathbf{c}_{\mathbf{0}}} \mathbf{E}_{\mathbf{c}_{-}} \mid \mathbf{c}_{+} \in \oplus_{i \in R_{r e}^{+}(>)} \mathbf{Z}_{\geq 0}^{(i)}, \mathbf{c}_{\mathbf{o}} \in \oplus_{n \geq 1} \mathbf{Z}_{\geq 0}^{(n)}, \mathbf{c}_{-} \in \oplus_{i \in R_{r e}^{+}(<)} \mathbf{Z}_{\geq 0}^{(i)}\right\}$,

(3) $\left\{\mathbf{E}_{\mathbf{c}_{+}} \mathbf{S}_{\lambda} \mathbf{E}_{\mathbf{c}_{-}} \mid \mathbf{c}_{+} \in \oplus_{i \in R_{r e}^{+}(>)} \mathbf{Z}_{\geq 0}^{(i)}, \lambda\right.$ is a partition, $\left.\mathbf{c}_{-} \in \oplus_{i \in R_{r e}^{+}(<)} \mathbf{Z}_{\geq 0}^{(i)}\right\}$.

Proof. This follows from Propositions 6.6 and 6.18 .

Remark 6.20. Corollary 4.7 (3), Proposition 4.17 ((2), (4)), and Proposition 4.19 (2) are referred to as the convexity of the bases in (1) and (2) of Theorem 6.19.

Lemma 6.21. The basis in Proposition 6.19 (3) is quasi-orthonormal with respect to the inner product; namely, for $\mathbf{c}_{+}, \mathbf{c}_{+}^{\prime} \in \oplus_{i \in R_{r e}^{+}(>)} \mathbf{Z}_{\geq 0}^{(i)}$, for $\mathbf{c}_{-}, \mathbf{c}_{-}^{\prime} \in \oplus_{i \in R_{r e}^{+}(<)} \mathbf{Z}_{\geq 0}^{(i)}$, and for partitions $\lambda$ and $\mu$, we have

$$
\left(\mathbf{E}_{\mathbf{c}_{+}} \mathbf{S}_{\lambda} \mathbf{E}_{\mathbf{c}_{-}}, \mathbf{E}_{\mathbf{c}_{+}^{\prime}} \mathbf{S}_{\mu} \mathbf{E}_{\mathbf{c}_{-}^{\prime}}\right) \equiv \delta_{\mathbf{c}_{+}, \mathbf{c}_{+}^{\prime}} \delta_{\lambda, \mu} \delta_{\mathbf{c}_{-}, \mathbf{c}_{-}^{\prime}} \bmod q_{1}^{-1} \mathbf{A} .
$$

Proof. This follows from Propositions 6.4 and 6.18 (2).

Definition 6.22. Let $n \geq 0$. In view of Proposition 6.18 (4), we set

$$
\begin{aligned}
& \mathbf{U}_{\mathbf{Z}}^{+}(0)=\mathbf{Z}\left[q_{1}, q_{1}^{-1}\right]\left[P_{1}, P_{2}, \cdots\right] \subset \mathbf{U}^{+}(0), \\
& \mathbf{U}_{\mathbf{Z}}^{+}(0 ; n)=\mathbf{Z}\left[q_{1}, q_{1}^{-1}\right]\left[P_{1}, \cdots, P_{n}\right] \subset \mathbf{U}^{+}(0 ; n) .
\end{aligned}
$$

We understand that $\mathbf{U}_{\mathbf{Z}}^{+}(0 ; 0)=\mathbf{Z}\left[q_{1}, q_{1}^{-1}\right]$. 
It follows from Lemma 4.11 that $\tilde{\psi}_{n} \in \mathbf{U}_{\mathbf{Z}}^{+}(0 ; n)$ for $n \geq 1$.

Lemma 6.23. The $\mathbf{S}_{\lambda}$ form a $\mathbf{Z}\left[q_{1}, q_{1}^{-1}\right]$-basis of $\mathbf{U}_{\mathbf{Z}}^{+}(0)$.

Proof. In the proof of Proposition 6.18, it is shown that the $\mathbf{S}_{\lambda}$ form a $\mathbf{Z}$ basis of $S$; thus, they span $\mathbf{U}_{\mathbf{Z}}^{+}(0)$ over $\mathbf{Z}\left[q_{1}, q_{1}^{-1}\right]$. Their linearly independence over $\mathbf{Z}\left[q_{1}, q_{1}^{-1}\right]$ follows from Proposition 6.18 (3).

Definition 6.24. We set $V_{\mathbf{Z}}=\mathbf{U}_{\mathbf{Z}}^{+}(>) \mathbf{U}_{\mathbf{Z}}^{+}(0) \mathbf{U}_{\mathbf{Z}}^{+}(<) \subset \mathbf{U}^{+}$.

It is clear that the basis in Theorem 6.19 (2) is a $\mathbf{Z}\left[q_{1}, q_{1}^{-1}\right]$-basis of $V_{\mathbf{Z}}$. By Lemma 6.23, the basis in Theorem 6.19 (3) is also a $\mathbf{Z}\left[q_{1}, q_{1}^{-1}\right]$-basis of $V_{\mathbf{Z}}$. We are going to prove that $\mathbf{U}_{\mathbf{Z}}^{+}(0) \subset \mathbf{U}_{\mathbf{Z}}^{+}$(hence $V_{\mathbf{Z}} \subset \mathbf{U}_{\mathbf{Z}}^{+}$) and that $V_{\mathbf{Z}}$ is closed under multiplication (Section 8). As a result of these, we conclude that $V_{\mathbf{Z}}=\mathbf{U}_{\mathbf{Z}}^{+}$, since $V_{\mathbf{Z}}$ contains the generators of the $\mathbf{Z}\left[q_{1}, q_{1}^{-1}\right]$-algebra $\mathbf{U}_{\mathbf{Z}}^{+}$; thus, we obtain $\mathbf{Z}\left[q_{1}, q_{1}^{-1}\right]$-bases of $\mathbf{U}_{\mathbf{Z}}^{+}$.

\section{$\S 7 . \quad$ Key Proposition}

In this section, $\equiv$ means the congruence modulo $\mathbf{U}^{0} \mathbf{U}^{+}(<)_{\leq-2} \otimes \mathbf{U}^{+}$, unless otherwise stated.

Definition 7.1. For $s, t \geq 0$, we define the elements $D_{s \alpha_{0}+t \alpha_{1}}^{-}$of $\mathbf{U}_{\mathbf{Z}}^{+}(<) \cap \mathbf{U}_{s \alpha_{0}+t \alpha_{1}}^{+}$by the induction on $s$ as follows:

$$
\begin{aligned}
D_{s \alpha_{0}+t \alpha_{1}}^{-}= & \sum_{k=[(t+2) / 2]}^{\min (t-1, s)} \sum_{l=t-k+1}^{\min (3(t-k), k)} T_{\varpi_{1}}\left(D_{(t-k) \alpha_{0}+(3(t-k)-l) \alpha_{1}}^{-}\right) E_{\delta-\alpha_{1}}^{(k-l)} E_{\alpha_{0}}^{(s-k)} \\
& \times q_{1}^{-(3(t-k)-l)(k-l)} q^{-2(t-k)(s-k)} \theta(s \geq 2) \theta(3 \leq t \leq 2 s-1) \\
& +E_{\delta-\alpha_{1}}^{(t)} E_{\alpha_{0}}^{(s-t)} \theta(s \geq t) .
\end{aligned}
$$

Note that if $s \geq 2,3 \leq t \leq 2 s-1,[(t+2) / 2] \leq k \leq \min (t-1, s), t-k+1 \leq$ $l \leq \min (3(t-k), k)$, we have $0 \leq t-k \leq s-1,3(t-k)-l \geq 0$; hence, the defining process by the induction on $s$ works.

Lemma 7.2. Let $s, t \geq 0$. Then,

(1) $D_{s \alpha_{0}}^{-}=E_{\alpha_{0}}^{(s)}$,

(2) $D_{t \alpha_{1}}^{-}=\delta_{t, 0}$,

(3) $D_{s \alpha_{0}+t \alpha_{1}}^{-}=0$ if $s \geq 1, t \geq 2 s$, 
(4) $D_{s \alpha_{0}+\alpha_{1}}^{-}=E_{\delta-\alpha_{1}} E_{\alpha_{0}}^{(s-1)}$ if $s \geq 1$,

(5) $D_{s \alpha_{0}+(2 s-1) \alpha_{1}}^{-}=E_{s \delta-\alpha_{1}}$ if $s \geq 1$.

Proof. This follows from Definition 7.1: (1)-(4) are clear and (5) is checked by the induction on $s$.

Definition 7.3. For $s \geq 1,1 \leq t \leq 2 s-1,1 \leq p \leq[(t+1) / 2]$, we define the elements $d_{s \alpha_{0}+t \alpha_{1}, p}$ of $\mathbf{U}_{\mathbf{Z}}^{+}(0) \mathbf{U}_{\mathbf{Z}}^{+}(<) \cap \mathbf{U}_{(s-p) \alpha_{0}+(t-2 p+1) \alpha_{1}}^{+}$by

$$
\begin{aligned}
& d_{s \alpha_{0}+t \alpha_{1}, p} \\
& \quad=\sum_{i=0}^{[(t-2 p+1) / 2]} \tilde{\psi}_{i} D_{(s-p-i) \alpha_{0}+(t-2 p-2 i+1) \alpha_{1}}^{-} q_{1}^{(-2 s+t)(2 p+2 i)+t+1}\left(q_{1}-q_{1}^{-1}\right) .
\end{aligned}
$$

Example 7.4. We have $d_{\alpha_{0}+\alpha_{1}, 1}=1$.

Let us study some properties of $D_{s \alpha_{0}+t \alpha_{1}}^{-}$.

Proposition 7.5. Let $s \geq 1$ and let $0 \leq t \leq 2 s-1$. Then,

(1) $D_{s \alpha_{0}+t \alpha_{1}}^{-}[t]_{1}$

$$
=\sum_{i=1}^{[(t+1) / 2]} E_{i \delta-\alpha_{1}} D_{(s-i) \alpha_{0}+(t-2 i+1) \alpha_{1}}^{-}[2 i-1]_{1} q^{(-2 s+t+1)(i-1)} \theta(t \geq 1),
$$

(2) $\left[D_{s \alpha_{0}+t \alpha_{1}}^{-}, E_{\alpha_{1}}\right]_{q^{-2 s+t}}$

$$
\begin{aligned}
= & D_{s \alpha_{0}+(t+1) \alpha_{1}}^{-}[t+1]_{1} \\
& +\sum_{i=1}^{[(t+1) / 2]} \tilde{\psi}_{i} D_{(s-i) \alpha_{0}+(t-2 i+1) \alpha_{1}}^{-} q_{1}^{(-2 s+t)(2 i+1)+2 s+1} \theta(t \geq 1),
\end{aligned}
$$

(3) $\Delta\left(D_{s \alpha_{0}+t \alpha_{1}}^{-}\right)$

$$
\begin{aligned}
\equiv & c^{s} k_{1}^{-2 s+t} \otimes D_{s \alpha_{0}+t \alpha_{1}}^{-} \\
& +\sum_{p=1}^{[(t+1) / 2]} c^{s-p} k_{1}^{-2 s+t+1} E_{p \delta-\alpha_{1}} \otimes d_{s \alpha_{0}+t \alpha_{1}, p} \theta(t \geq 1),
\end{aligned}
$$

(4) $\Delta\left(T_{\varpi_{1}}\left(D_{s \alpha_{0}+t \alpha_{1}}^{-}\right)\right)$

$$
\begin{aligned}
\equiv & c^{3 s-t} k_{1}^{-2 s+t} \otimes T_{\varpi_{1}}\left(D_{s \alpha_{0}+t \alpha_{1}}^{-}\right) \\
& +\sum_{p=2}^{[(t+3) / 2]} c^{3 s-t-p} k_{1}^{-2 s+t+1} E_{p \delta-\alpha_{1}} \otimes T_{\varpi_{1}}\left(d_{s \alpha_{0}+t \alpha_{1}, p-1}\right) \theta(t \geq 1) \\
& +c^{3 s-t-1} k_{1}^{-2 s+t+1} E_{\delta-\alpha_{1}} \otimes T_{\varpi_{1}}\left(\left[D_{s \alpha_{0}+t \alpha_{1}}^{-}, E_{\alpha_{1}}\right]_{q^{-2 s+t}}\right)\left(q_{1}-q_{1}^{-1}\right),
\end{aligned}
$$


(5) $\left[E_{\delta-\alpha_{1}}, T_{\varpi_{1}}\left(D_{s \alpha_{0}+t \alpha_{1}}^{-}\right)\right]_{q^{2 s-t}}$

$$
\begin{aligned}
= & T_{\varpi_{1}}\left(D_{s \alpha_{0}+(t-1) \alpha_{1}}^{-}\right)[4 s-t+1]_{1} \theta(t \geq 1) \\
& -\sum_{i=1}^{[t / 2]} E_{(i+1) \delta-\alpha_{i}} T_{\varpi_{1}}\left(D_{(s-i) \alpha_{0}+(t-2 i) \alpha_{1}}^{-}\right)[2 i+1]_{1} q^{(-2 s+t) i} \theta(t \geq 2) .
\end{aligned}
$$

Proof. We denote by $(\text { a })_{r}$ the statement (a) for $s=1, \ldots, r$. We prove $(1)_{s}-(5)_{s}$ at once by the induction on $s$. If $s=1$, we have (1) clearly, (2) and (5) by Corollary 3.10, and (3) and (4) by Lemma 5.3 and Lemma 5.4. Now, assume that $s \geq 2$.

$(1)_{s}$ We use $(5)_{s-1}$. We rewrite the the right hand side of (1), which is denoted by RHS. By Definition 7.1, we have

$$
\begin{aligned}
\mathrm{RHS}= & \sum_{i=1}^{[(t+1) / 2]} E_{i \delta-\alpha_{1}}\left(\sum_{k=[(t-2 i+3) / 2]}^{\min (t-2 i, s-i)} \sum_{l=t-2 i-k+2}^{\min (3(t-2 i-k+1), k)}\right. \\
& T_{\varpi_{1}}\left(D_{(t-2 i-k+1) \alpha_{0}+(3(t-2 i-k+1)-l) \alpha_{1}}^{-}\right) E_{\delta-\alpha_{1}}^{(k-l)} E_{\alpha_{0}}^{(s-i-k)} \\
& \times q_{1}^{-(3(t-2 i-k+1)-l)(k-l)} q^{-2(t-2 i-k+1)(s-i-k)} \\
& \times \theta(s-i \geq 2) \theta(3 \leq t-2 i+1 \leq 2(s-i)-1) \\
& \left.+E_{\delta-\alpha_{1}}^{(t-2 i+1)} E_{\alpha_{0}}^{(s-t+i-1)} \theta(s-t+i-1 \geq 0)\right)[2 i-1]_{1} q^{(-2 s+t+1)(i-1)} .
\end{aligned}
$$

By $(5)_{s-1}$, the $i=1$ part is equal to

$$
\begin{aligned}
& \sum_{k=[(t+1) / 2]}^{\min (t-2, s-1)} \sum_{l=t-k}^{\min (3(t-k-1), k)}\left(T_{\varpi_{1}}\left(D_{(t-k-1) \alpha_{0}+(3(t-k-1)-l) \alpha_{1}}^{-}\right) E_{\delta-\alpha_{1}} q^{-t+k+l+1}\right. \\
& +T_{\varpi_{1}}\left(D_{(t-k-1) \alpha_{0}+(3(t-k-1)-l-1) \alpha_{1}}^{-}\right)[t-k+l]_{1} \theta(3(t-k-1)-l \geq 1) \\
& \quad-\sum_{i=1}^{[(3(t-k-1)-l) / 2]} E_{(i+1) \delta-\alpha_{1}} T_{\varpi_{1}}\left(D_{(t-k-i-1) \alpha_{0}+(3(t-k-1)-l-2 i) \alpha_{1}}^{-}\right) \\
& \left.\times[2 i+1]_{1} q^{(t-k-l-1) i} \theta(3(t-k-1)-l \geq 2)\right) E_{\delta-\alpha_{1}}^{(k-l)} E_{\alpha_{0}}^{(s-k-1)} \\
& \times q_{1}^{-(3(t-k-1)-l)(k-l)} q^{-2(t-k-1)(s-k-1)} \theta(s \geq 3) \theta(4 \leq t \leq 2 s-2) \\
& +E_{\delta-\alpha_{1}}^{(t)} E_{\alpha_{0}}^{(s-t)}[t]_{1} \theta(s \geq t) .
\end{aligned}
$$

Hence, if we set

$$
A=\sum_{k=[(t+1) / 2]}^{\min (t-2, s-1)} \sum_{l=t-k}^{\min (3(t-k-1), k)} T_{\varpi_{1}}\left(D_{(t-k-1) \alpha_{0}+(3(t-k-1)-l) \alpha_{1}}^{-}\right)
$$




$$
\begin{aligned}
& \times E_{\delta-\alpha_{1}}^{(k-l+1)} E_{\alpha_{0}}^{(s-k-1)}[k-l+1]_{1} q^{-t+k+l+1} \\
& \times q_{1}^{-(3(t-k-1)-l)(k-l)} q^{-2(t-k-1)(s-k-1)} \theta(s \geq 3) \theta(4 \leq t \leq 2 s-2), \\
& B=\sum_{k=[(t+1) / 2]}^{\min (t-2, s-1)} \sum_{l=t-k}^{\min (3(t-k-1)} \\
& T_{\varpi_{1}}\left(D_{(t-k-1) \alpha_{0}+(3(t-k-1)-l-1) \alpha_{1}}^{-}\right) E_{\delta-\alpha_{1}}^{(k-l)} E_{\alpha_{0}}^{(s-k-1)} \\
& \times[t-k+l]_{1} q_{1}^{-(3(t-k-1)-l)(k-l)} q^{-2(t-k-1)(s-k-1)} \\
& \times \theta(s \geq 3) \theta(4 \leq t \leq 2 s-2), \\
& C=-\sum_{k=[(t+1) / 2]}^{\min (t-3, s-1)} \sum_{l=t-k}^{\min (3(t-k-1)-2, k)} \sum_{i=1}^{[(3(t-k-1)-l) / 2]} \\
& E_{(i+1) \delta-\alpha_{1}} T_{\varpi_{1}}\left(D_{(t-k-i-1) \alpha_{0}+(3(t-k-1)-l-2 i) \alpha_{1}}^{-}\right) E_{\delta-\alpha_{1}}^{(k-l)} E_{\alpha_{0}}^{(s-k-1)} \\
& \times[2 i+1]_{1} q_{1}^{-(3(t-k-1)-l)(k-l)} q^{-2(t-k-1)(s-k-1)} q^{(t-k-l-1) i} \\
& \times \theta(s \geq 4) \theta(6 \leq t \leq 2 s-2), \\
& {[(t+1) / 2] \min (t-2 i, s-i) \min (3(t-2 i+1-k), k)} \\
& D=\sum_{i=2} \sum_{k=[(t-2 i+3) / 2]} \sum_{l=t-2 i-k+2} \\
& E_{i \delta-\alpha_{1}} T_{\varpi_{1}}\left(D_{(t-2 i-k+1) \alpha_{0}+(3(t-2 i-k+1)-l) \alpha_{1}}^{-}\right) E_{\delta-\alpha_{1}}^{(k-l)} E_{\alpha_{0}}^{(s-i-k)} \\
& \times[2 i-1]_{1} q_{1}^{-(3(t-2 i-k+1)-l)(k-l)} q^{-2(t-2 i-k+1)(s-i-k)} q^{(-2 s+t+1)(i-1)}, \\
& E=\sum_{i=2}^{[(t+1) / 2]} E_{i \delta-\alpha_{1}} E_{\delta-\alpha_{1}}^{(t-2 i+1)} E_{\alpha_{0}}^{(s-t+i-1)} \\
& \times[2 i-1]_{1} q^{(-2 s+t+1)(i-1)} \theta(s-t+i-1 \geq 0) \theta(t \geq 3),
\end{aligned}
$$

then we have

$$
\mathrm{RHS}=A+B+C+D+E+E_{\delta-\alpha_{1}}^{(t)} E_{\alpha_{0}}^{(s-t)} \theta(s \geq t)[t]_{1} .
$$

We rewrite $A$. Putting $k-l+1=k^{\prime}-l^{\prime}, s-k-1=s-k^{\prime}$, we see that $l=l^{\prime}, k=$ $k^{\prime}-1$ and that $[(t+1) / 2] \leq k \leq \min (t-2, s-1), t-k \leq l \leq \min (3(t-k-1), k)$ is equivalent to $[(t+3) / 2] \leq k^{\prime} \leq \min (t-1, s), t-k^{\prime}+1 \leq l^{\prime} \leq \min \left(3\left(t-k^{\prime}\right), k^{\prime}-1\right)$. Hence,

$$
\begin{aligned}
A= & \sum_{k=[(t+3) / 2]}^{\min (t-1, s)} \sum_{l=t-k+1}^{\min (3(t-k), k-1)} T_{\varpi_{1}}\left(D_{(t-k) \alpha_{0}+(3(t-k)-l) \alpha_{1}}^{-}\right) E_{\delta-\alpha_{1}}^{(k-l)} E_{\alpha_{0}}^{(s-k)} \\
& \times[k-l]_{1} q^{-t+k+l} q_{1}^{-(3(t-k)-l)(k-l-1)} q^{-2(t-k)(s-k)} \\
& \quad \times \theta(s \geq 3) \theta(4 \leq t \leq 2 s-2) .
\end{aligned}
$$


We rewrite $B$. Putting $k-l=k^{\prime}-l^{\prime}, s-k-1=s-k^{\prime}$, we see that $l=l^{\prime}-1, k=$ $k^{\prime}-1$ and that $[(t+1) / 2] \leq k \leq \min (t-2, s-1), t-k \leq l \leq \min (3(t-k-1)-1, k)$ is equivalent to $[(t+3) / 2] \leq k^{\prime} \leq \min (t-1, s), t-k^{\prime}+2 \leq l^{\prime} \leq \min \left(3\left(t-k^{\prime}\right), k^{\prime}\right)$.

Hence,

$$
\begin{aligned}
B= & \sum_{k=[(t+3) / 2]}^{\min (t-1, s)} \sum_{l=t-k+2}^{\min (3(t-k), k-1)} T_{\varpi_{1}}\left(D_{(t-k) \alpha_{0}+(3(t-k)-l) \alpha_{1}}^{-}\right) E_{\delta-\alpha_{1}}^{(k-l)} E_{\alpha_{0}}^{(s-k)} \\
& \times[t-k+l]_{1} q_{1}^{-(3(t-k)-l+1)(k-l)} q^{-2(t-k)(s-k)} \theta(s \geq 3) \theta(4 \leq t \leq 2 s-2) .
\end{aligned}
$$

We rewrite $D$. Putting $i=i^{\prime}+1, t-2 i-k+1=t-k^{\prime}-i^{\prime}-1,3(t-2 i-$ $k+1)-l=3\left(t-k^{\prime}-1\right)-l^{\prime}-2 i^{\prime}$, we see that $k=k^{\prime}-i^{\prime}, l=l^{\prime}-i^{\prime}$ and that $2 \leq i \leq[(t-2) / 2],[(t-2 i+3) / 2] \leq k \leq \min (t-2 i, s-i), t-2 i-k+2 \leq l \leq$ $\min (3(t-2 i-k+1), k)$ is equivalent to $[(t+1) / 2] \leq k^{\prime} \leq \min (t-3, s-1), t-k^{\prime} \leq$ $l^{\prime} \leq \min \left(3\left(t-k^{\prime}-1\right)-2, k^{\prime}\right), 1 \leq i^{\prime} \leq\left[\left(3\left(t-k^{\prime}-1\right)-l^{\prime}\right) / 2\right]$. Hence,

$$
\begin{aligned}
D= & \sum_{k=[(t+1) / 2]}^{\min (t-3, s-1)} \sum_{l=t-k}^{\min (3(t-k-1)-2, k)[(3(t-k-1)-l) / 2]} \sum_{i=1}{ }^{E_{(i+1) \delta-\alpha_{1}} T_{\varpi_{1}}\left(D_{(t-k-i-1) \alpha_{0}+(3(t-k+1)-l-2 i) \alpha_{1}}^{-}\right) E_{\delta-\alpha_{1}}^{(k-l)} E_{\alpha_{0}}^{(s-k-1)}} \\
& \times[2 i+1]_{1} q_{1}^{-(3(t-k-i-1)-l+i)(k-l)} q^{-2(t-k-i-1)(s-k-1)} q^{(-2 s+t+1) i} \\
& \times \theta(s \geq 4) \theta(6 \leq t \leq 2 s-2),
\end{aligned}
$$

which cancels out with $C$. We rewrite $E$. Putting $t-2 i+1=k^{\prime}-l^{\prime}, s-t+i$ $-1=s-k^{\prime}$, we see that $i=l^{\prime}=t-k^{\prime}+1$ and that $2 \leq i \leq[(t+1) / 2], s-t+i-1$ is equivalent to $[(t+2) / 2] \leq k^{\prime} \leq \min (t-1, s)$. Hence,

$$
\begin{aligned}
E= & \sum_{k=[(t+2) / 2]}^{\min (t-1, s)} T_{\varpi_{1}}\left(D_{(t-k) \alpha_{0}+(3(t-k)-l) \alpha_{1}}^{-}\right) E_{\delta-\alpha_{1}}^{(k-l)} E_{\alpha_{0}}^{(s-k)} \\
& \times[2 l-1]_{1} q^{(l-1)(-2 s+t+1)} \theta(k+l=t+1) \theta(s \geq 2) \theta(3 \leq t \leq 2 s-1) .
\end{aligned}
$$

It is now easy to see that

$$
\begin{aligned}
A+B+E= & \sum_{k=[(t+2) / 2]}^{\min (t-1, s)} \sum_{l=t-k+1}^{\min (3(t-k), k)} T_{\varpi_{1}}\left(D_{(t-k) \alpha_{0}+(3(t-k)-l) \alpha_{1}}^{-}\right) E_{\delta-\alpha_{1}}^{(k-l)} E_{\alpha_{0}}^{(s-k)} \\
& \times q_{1}^{-(3(t-k)-l)(k-l)} q^{-2(t-k)(s-k)} \theta(s \geq 2) \theta(3 \leq t \leq 2 s-1)[t]_{1} .
\end{aligned}
$$

Thus, RHS $=D_{s \alpha_{0}+t \alpha_{1}}^{-}[t]_{1}$. We obtain (1) for $s$.

$(2)_{s}$ We use $(1)_{s}$. The case where $t=0$ is directly checked by the induction on $s$ in view of Lemma $7.2(5)$. The case where $t=2 s-1$ is clear. We assume 
that $s \geq 2,1 \leq t \leq 2 s-2$. We rewrite the left hand side, which is denoted by LHS. By $(1)_{s}$, we have

\section{$\operatorname{LHS}[t]_{1}$}

$$
=\sum_{l=1}^{[(t+1) / 2]}\left[E_{l \delta-\alpha_{1}} D_{(s-l) \alpha_{0}+(t-2 l+1) \alpha_{1}}^{-}, E_{\alpha_{1}}\right]_{q^{-2 s+t}}[2 l-1]_{1} q^{(-2 s+t+1)(l-1)} .
$$

For $1 \leq l \leq[(t+1) / 2]$, using Lemma 2.11, $(2)_{s-1}$, and Proposition $3.26((2)$, $(5))$, we have

$$
\begin{aligned}
& {\left[E_{l \delta-\alpha_{1}} D_{(s-l) \alpha_{0}+(t-2 l+1) \alpha_{1}}^{-}, E_{\alpha_{1}}\right]_{q^{-2 s+t}}} \\
& =E_{l \delta-\alpha_{1}}\left[D_{(s-l) \alpha_{0}+(t-2 l+1) \alpha_{1}}^{-}, E_{\alpha_{1}}\right]_{q^{-2 s+t+1}} \\
& \quad+\left[E_{l \delta-\alpha_{1}}, E_{\alpha_{1}}\right]_{q^{-1}} D_{(s-l) \alpha_{0}+(t-2 l+1) \alpha_{1}}^{-} q^{-2 s+t+1} \\
& =\tilde{\psi}_{l} D_{(s-l) \alpha_{0}+(t-2 l+1) \alpha_{1}}^{-} q^{-2 s+t+1}+E_{l \delta-\alpha_{1}} D_{(s-l) \alpha_{0}+(t-2 l+2) \alpha_{1}}^{-}[t-2 l+2]_{1} \\
& \quad+\sum_{i=1}^{[(t-2 l+2) / 2]}\left(\tilde{\psi}_{i} E_{l \delta-\alpha_{1}}+\left(1+q^{-1}\right) \sum_{j=0}^{i-1} \tilde{\psi}_{j} E_{(l+i-j) \delta-\alpha_{1}} b_{2(i-j)}\right) \\
& \quad+D_{(s-l-i) \alpha_{0}+(t-2 l-2 i+2) \alpha_{1}}^{-} q_{1}^{(-2 s+t+1)(2 i+1)+2(s-l)+1} \theta(t \geq 2 l) .
\end{aligned}
$$

Hence, if we set

$$
\begin{aligned}
A= & \sum_{l=1}^{[(t+1) / 2]} \tilde{\psi}_{l} D_{(s-l) \alpha_{0}+(t-2 l+1) \alpha_{1}}^{-}[2 l-1]_{1} q^{(-2 s+t+1) l}, \\
B= & \sum_{l=1}^{[(t+1) / 2]} E_{l \delta-\alpha_{1}} D_{(s-l) \alpha_{0}+(t-2 l+2) \alpha_{1}}^{-}[t-2 l+2]_{1}[2 l-1]_{1} q^{(-2 s+t+1)(l-1)}, \\
C= & \sum_{l=1}^{[t / 2]} \sum_{i=1}^{[(t-2 l+2) / 2]} \tilde{\psi}_{i} E_{l \delta-\alpha_{1}} D_{(s-l-i) \alpha_{0}+(t-2 l-2 i+2) \alpha_{1}}^{-} \\
& \times[2 l-1]_{1} q^{(-2 s+t+1)(l+i-1)} q_{1}^{t-2 l+2}, \\
D= & \sum_{l=1}^{[t / 2][(t-2 l+2) / 2]} \sum_{i=1}^{i-1} \sum_{j=0}^{i} \tilde{\psi}_{j} E_{(l+i-j) \delta-\alpha_{1}} D_{(s-l-i) \alpha_{0}+(t-2 l-2 i+2) \alpha_{1}}^{-} \\
& \times b_{2(i-j)}[2 l-1]_{1}\left(1+q^{-1}\right) q^{(-2 s+t+1)(l+i-1)} q_{1}^{t-2 l+2},
\end{aligned}
$$

then we have

$$
\operatorname{LHS}[t]_{1}=A+B+C+D \text {. }
$$


In the above expression of $B$, on account of the factor $[t-2 l+2]_{1}$, we can include the case where $l=[(t+2) / 2]$. We rewrite $C$. Since $1 \leq l \leq[t / 2], 1 \leq$ $i \leq[(t-2 l+2) / 2]$ is equivalent to $1 \leq i \leq[t / 2], 1 \leq l \leq[(t-2 i+2) / 2]$, we have

$$
\begin{aligned}
C= & \sum_{i=1}^{[t / 2]} \sum_{l=1}^{[(t-2 i+2) / 2]} \tilde{\psi}_{i} E_{l \delta-\alpha_{1}} D_{(s-l-i) \alpha_{0}+(t-2 i-2 l+2) \alpha_{1}}^{-} \\
& \times[2 l-1]_{1} q^{(-2 s+t+1)(i+l-1)} q_{1}^{t-2 l+2} \theta(t \geq 2) .
\end{aligned}
$$

We rewrite $D$. Putting $j=i^{\prime}, l+i-j=l^{\prime}, i-j=j^{\prime}$, we see that $i=$ $i^{\prime}+j^{\prime}, l=l^{\prime}-j^{\prime}$ and that $1 \leq l \leq[t / 2], 1 \leq i \leq[(t-2 l+2) / 2], 0 \leq j \leq i-1$ is equivalent to $0 \leq i^{\prime} \leq[(t-2) / 2], 2 \leq l^{\prime} \leq\left[\left(t-2 i^{\prime}+2\right) / 2\right], 1 \leq j \leq l^{\prime}-1$. Hence, using Lemma 3.17 (1), we have

$$
\begin{aligned}
D= & \sum_{i=0}^{[(t-2) / 2]} \sum_{l=2}^{[(t-2 i+2) / 2]} \sum_{j=1}^{l-1} \tilde{\psi}_{i} E_{l \delta-\alpha_{1}} D_{(s-l-i) \alpha_{0}+(t-2 i-2 l+2) \alpha_{1}}^{-} \\
& \times[2 l-2 j-1]_{1}\left(1+q^{-1}\right) b_{2 j} q^{(-2 s+t+1)(i+l-1)+j} q_{1}^{t-2 l+2} \theta(t \geq 2) \\
= & \sum_{i=0}^{[(t-2) / 2]} \sum_{l=2}^{[(t-2 i+2) / 2]} \tilde{\psi}_{i} E_{l \delta-\alpha_{1}} D_{(s-l-i) \alpha_{0}+(t-2 i-2 l+2) \alpha_{1}}^{-} \\
& \times[2 l-2]_{1}[2 l-1]_{1}\left(q_{1}-q_{1}^{-1}\right) q^{(-2 s+t+1)(i+l-1)} q_{1}^{t} \theta(t \geq 2) .
\end{aligned}
$$

Here, on account of the factor $[2 l-2]_{1}$, we can include the case where $l=1$; hence, we can also include the case where $i=[t / 2]$ and thus the case where $t=1$. Thus,

$$
\begin{aligned}
D= & \sum_{i=0}^{[t / 2]} \sum_{l=1}^{[(t-2 i+2) / 2]} \tilde{\psi}_{i} E_{l \delta-\alpha_{1}} D_{(s-l-i) \alpha_{0}+(t-2 i-2 l+2) \alpha_{1}}^{-} \\
& \times[2 l-1]_{1} q^{(-2 s+t+1)(i+l-1)} q_{1}^{t}\left(q_{1}^{2 l-2}-q_{1}^{-2 l+2}\right),
\end{aligned}
$$

the $i=0$ part of which is denoted by $E$. Then,

$$
\begin{aligned}
C+(D-E)= & \sum_{i=1}^{[t / 2]} \sum_{l=1}^{[(t-2 i+2) / 2]} \tilde{\psi}_{i} E_{l \delta-\alpha_{1}} D_{(s-l-i) \alpha_{0}+(t-2 i-2 l+2) \alpha_{1}}^{-} \\
& \times[2 l-1]_{1} q^{(-2 s+t+1)(i+l-1)} q_{1}^{t+2 l-2} \theta(t \geq 2) .
\end{aligned}
$$

Applying (1) $)_{s-1}$, we have

$$
C+(D-E)=\sum_{i=1}^{[t / 2]} \tilde{\psi}_{i} D_{(s-i) \alpha_{0}+(t-2 i+1) \alpha_{1}}^{-}[t-2 i+1]_{1} q_{1}^{t-2 i}
$$


where the range of $i$ can be changed to $1 \leq i \leq[(t+1) / 2]$ on account of the factor $[t-2 i+1]_{1}$. Thus,

$$
A+C+(D-E)=\sum_{i=1}^{[(t+1) / 2]} \tilde{\psi}_{i} D_{(s-i) \alpha_{0}+(t-2 i+1) \alpha_{1}}^{-}[t]_{1} q^{(-2 s+t) i} q_{1}^{t+1}
$$

On the other hand, by $(1)_{s}$, we have

$$
\begin{aligned}
B+E & =\sum_{l=1}^{[(t+2) / 2]} E_{l \delta-\alpha_{1}} D_{(s-l) \alpha_{0}+(t-2 l+2) \alpha_{1}}^{-}[2 l-1]_{1}[t]_{1} q^{(-2 s+t+2)(l-1)} \\
& =D_{s \alpha_{0}+(t+1) \alpha_{1}}^{-}[t+1]_{1}[t]_{1}
\end{aligned}
$$

Hence, we obtain (2) for $s$.

$(3)_{s}$ We use $(1)_{s}$. We can assume that $t \geq 1$. By $(1)_{s}$, we have

$$
\begin{aligned}
\Delta\left(D_{s \alpha_{0}+t \alpha_{1}}^{-}\right)[t]_{1}= & \sum_{l=1}^{[(t+1) / 2]} \Delta\left(E_{l \delta-\alpha_{1}} D_{(s-l) \alpha_{0}+(t-2 l+1) \alpha_{1}}^{-}\right) \\
& \times[2 l-1]_{1} q^{(-2 s+t+1) l}\left(q_{1}-q_{1}^{-1}\right) .
\end{aligned}
$$

For $1 \leq l \leq[(t+1) / 2]$, using Lemma 5.3, $(3)_{s-1}$, and Corollary 4.7, we have

$$
\begin{aligned}
\Delta & \left(E_{l \delta-\alpha_{1}} D_{(s-l) \alpha_{0}+(t-2 l+1) \alpha_{1}}^{-}\right) \\
\equiv & \left(c^{l} k_{1}^{-1} \otimes E_{l \delta-\alpha_{1}}+\left(q_{1}-q_{1}^{-1}\right) \sum_{i=1}^{l} c^{l-i} E_{i \delta-\alpha_{1}} \otimes \tilde{\psi}_{l-i}\right) \\
& \times\left(c^{s-l} k_{1}^{-2 s+t+1} \otimes D_{(s-l) \alpha_{0}+(t-2 l+1) \alpha_{1}}^{-}\right. \\
& \left.+\sum_{p=1}^{[(t-2 l+2) / 2]} c^{s-l-p} k_{1}^{-2 s+t+2} E_{p \delta-\alpha_{1}} \otimes d_{(s-l) \alpha_{0}+(t-2 l+1) \alpha_{1}, p} \theta(t \geq 2 l)\right) \\
\equiv & c^{s} k_{1}^{-2 s+t} \otimes E_{l \delta-\alpha_{1}} D_{(s-l) \alpha_{0}+(t-2 l+1) \alpha_{1}}^{-} \\
& +\sum_{i=1}^{l} c^{s-i} k_{1}^{-2 s+t+1} E_{i \delta-\alpha_{1}} \otimes \tilde{\psi}_{l-i} D_{(s-l) \alpha_{0}+(t-2 l+1) \alpha_{1}}^{-} q^{-2 s+t+1}\left(q_{1}-q_{1}^{-1}\right) \\
& +\sum_{p=1}^{[(t-2 l+2) / 2]} c^{s-p} k_{1}^{-2 s+t+1} E_{p \delta-\alpha_{1}} \otimes E_{l \delta-\alpha_{1}} d_{(s-l) \alpha_{0}+(t-2 l+1) \alpha_{1}, p} \theta(t \geq 2 l) .
\end{aligned}
$$


Hence, if we set

$$
\begin{aligned}
A= & \sum_{l=1}^{[(t+1) / 2]} \sum_{i=1}^{l} c^{s-i} k_{1}^{-2 s+t+1} E_{i \delta-\alpha_{1}} \otimes \tilde{\psi}_{l-i} D_{(s-l) \alpha_{0}+(t-2 l+1) \alpha_{1}}^{-} \\
& \times[2 l-1]_{1} q^{(-2 s+t+1) l}\left(q_{1}-q_{1}^{-1}\right), \\
B= & \sum_{l=1}^{[t / 2]} \sum_{p=1}^{[(t-2 l+2) / 2]} c^{s-p} k_{1}^{-2 s+t+1} E_{p \delta-\alpha_{1}} \otimes E_{l \delta-\alpha_{1}} d_{(s-l) \alpha_{0}+(t-2 l+1) \alpha_{1}, p} \\
& \times[2 l-1]_{1} q^{(-2 s+t+1)(l-1)} \theta(t \geq 2),
\end{aligned}
$$

then we have

$$
\Delta\left(D_{s \alpha_{0}+t \alpha_{1}}^{-}\right)[t]_{1} \equiv c^{s} k_{1}^{-2 s+t} \otimes D_{s \alpha_{0}+t \alpha_{1}}^{-}[t]_{1}+A+B .
$$

We rewrite $A$. Putting $i=p, l-i=i^{\prime}$, we see that $l=p+i^{\prime}$ and that $1 \leq l \leq$ $[(t+1) / 2], 1 \leq i \leq l$ is equivalent to $1 \leq p \leq[(t+1) / 2], 0 \leq i \leq[(t-2 p+1) / 2]$.

Hence,

$$
A=\sum_{p=1}^{[(t+1) / 2]} c^{s-p} k_{1}^{-2 s+t+1} E_{p \delta-\alpha_{1}} \otimes A_{p}
$$

where we set

$$
\begin{aligned}
A_{p}= & \sum_{i=0}^{[(t-2 p+1) / 2]} \tilde{\psi}_{i} D_{(s-p-i) \alpha_{0}+(t-2 p-2 i+1) \alpha_{1}}^{-} \\
& \times[2 p+2 i-1]_{1} q^{(-2 s+t+1)(p+i)}\left(q_{1}-q_{1}^{-1}\right) .
\end{aligned}
$$

We rewrite $B$. Since $1 \leq l \leq[t / 2], 1 \leq p \leq[(t-2 l+2) / 2]$ is equivalent to $1 \leq p \leq[t / 2], 1 \leq l \leq[(t-2 p+2) / 2]$, we have

$$
B=\sum_{p=1}^{[t / 2]} c^{s-p} k_{1}^{-2 s+t+1} E_{p \delta-\alpha_{1}} \otimes B_{p}
$$

where we set

$$
B_{p}=\sum_{l=1}^{[(t-2 p+2) / 2]} E_{l \delta-\alpha_{1}} d_{(s-l) \alpha_{0}+(t-2 l+1) \alpha_{1}, p}[2 l-1]_{1} q^{(-2 s+t+1)(l-1)} \theta(t \geq 2) .
$$

Note that if $t$ is odd and $p=(t+1) / 2$, then $A_{p}=D_{(s-p) \alpha_{0}}^{-}[t]_{1} q^{(-2 s+t+1) p}=$ $d_{s \alpha_{0}+t \alpha_{1}, p}[t]_{1}$. Hence, the proof of (3) for $s$ is reduced to showing that $A_{p}+B_{p}=$ 
$d_{s \alpha_{0}+t \alpha_{1}}[t]_{1}$ for $t \geq 2,1 \leq p \leq[t / 2]$. We rewrite $B_{p}$. Using Proposition 3.26 (5), we have

$$
\begin{aligned}
B_{p}= & \sum_{l=1}^{[(t-2 p+2) / 2]} \sum_{i=0}^{[(t-2 p-2 l+2) / 2]} E_{l \delta-\alpha_{1}} \tilde{\psi}_{i} D_{(s-l-p-i) \alpha_{0}+(t-2 p-2 l-2 i+2) \alpha_{1}}^{-} \\
& \times[2 l-1]_{1} q_{1}^{(-2 s+t+1)(2 p+2 i)+t-2 l+2} q^{(-2 s+t+1)(l-1)}\left(q_{1}-q_{1}^{-1}\right) \\
= & \sum_{l=1}^{[(t-2 p+2) / 2]} \sum_{i=0}^{[(t-2 p-2 l+2) / 2]}\left(\tilde{\psi}_{i} E_{l \delta-\alpha_{1}}\right. \\
& \left.+\left(1+q^{-1}\right) \sum_{j=0}^{i-1} b_{2(i-j)} \tilde{\psi}_{j} E_{(l+i-j) \delta-\alpha_{1}} \theta(i \geq 1)\right) \\
& \times D_{(s-p-l-i) \alpha_{0}+(t-2 p-2 l-2 i+2) \alpha_{1}}^{-} \\
& \times[2 l-1]_{1} q^{(-2 s+t+1)(p+l+i-1)} q_{1}^{t-2 l+2}\left(q_{1}-q_{1}^{-1}\right)
\end{aligned}
$$

In the double summation, $1 \leq l \leq[(t-2 p+2) / 2], 0 \leq i \leq[(t-2 p-2 l+2) / 2]$ is equivalent to $0 \leq i \leq[(t-2 p) / 2], 1 \leq l \leq[(t-2 p-2 i+2) / 2]$. In the triple one, putting $j=i^{\prime}, l+i-j=l^{\prime}, i-j=j^{\prime}$, we see that $i=i^{\prime}+j^{\prime}, l=l^{\prime}-j^{\prime}$ and that $1 \leq l \leq[(t-2 p+2) / 2], 1 \leq i \leq[(t-2 l-2 p+2) / 2], 0 \leq j \leq i-1$ is equivalent to $0 \leq i^{\prime} \leq[(t-2 p-2) / 2], 2 \leq l^{\prime} \leq\left[\left(t-2 p-2 i^{\prime}+2\right) / 2\right], 1 \leq j \leq l^{\prime}-1$. Thus,

$$
\begin{aligned}
B_{p}= & \sum_{i=0}^{[(t-2 p) / 2]} \sum_{l=1}^{[(t-2 p-2 i+2) / 2]} \tilde{\psi}_{i} E_{l \delta-\alpha_{1}} D_{(s-l-p-i) \alpha_{0}+(t-2 l-2 p-2 i+2) \alpha_{1}}^{-} \\
& \times[2 l-1]_{1} q^{(-2 s+t+1)(p+l+i-1)} q_{1}^{t-2 l+2}\left(q_{1}-q_{1}^{-1}\right) \\
& +\sum_{i=0}^{[(t-2 p-2) / 2]} \sum_{l=2}^{[(t-2 p-2 i) / 2]} \sum_{j=1}^{l-1} \tilde{\psi}_{i} E_{l \delta-\alpha_{1}} D_{(s-p-l-i) \alpha_{0}+(t-2 p-2 l-2 i+2) \alpha_{1}}^{-} \\
& \times[2 l-2 j-1]_{1} b_{2 j} q^{(-2 s+t+1)(p+i+l-1)+j} q_{1}^{t-2 l+2}\left(1+q^{-1}\right)\left(q_{1}-q_{1}^{-1}\right) .
\end{aligned}
$$

By Lemma 3.17, the latter term is equal to

$$
\begin{aligned}
& \sum_{i=0}^{[(t-2 p-2) / 2]} \sum_{l=2}^{[(t-2 p-2 i) / 2]} \tilde{\psi}_{i} E_{l \delta-\alpha_{1}} D_{(s-p-l-i) \alpha_{0}+(t-2 p-2 l-2 i+2) \alpha_{1}}^{-} \\
& \quad \times[2 l-1]_{1}\left(q^{l-1}-q^{-l+1}\right) q^{l-1} q^{(-2 s+t+1)(p+i+l-1)} q_{1}^{t-2 l+2}\left(q_{1}-q_{1}^{-1}\right) .
\end{aligned}
$$

Here, on account of the factor $\left(q^{l-1}-q^{-l+1}\right)$, we can include the case where $l=1$; hence, we can also include the case where $i=[(t-2 p-2) / 2]+1=$ 
$[(t-2 p) / 2]$. Thus,

$$
\begin{aligned}
B_{p}= & \sum_{i=0}^{[(t-2 p) / 2]} \sum_{l=1}^{[(t-2 p-2 i+2) / 2]} \tilde{\psi}_{i} E_{l \delta-\alpha_{1}} D_{(s-p-l-i) \alpha_{0}+(t-2 p-2 l-2 i+2) \alpha_{1}}^{-} \\
& \times[2 l-1]_{1} q^{(-2 s+t+1)(p+i+l-1)} q_{1}^{t+2 l-2}\left(q_{1}-q_{1}^{-1}\right) .
\end{aligned}
$$

Applying (1) $)_{s-1}$, we have

$$
\begin{aligned}
B_{p}= & \sum_{i=0}^{[(t-2 p) / 2]} \tilde{\psi}_{i} D_{(s-p-i) \alpha_{0}+(t-2 p-2 i+1) \alpha_{1}}^{-} \\
& \times[t-2 p-2 i+1]_{1} q^{(-2 s+t+1)(p+i)} q_{1}^{t}\left(q_{1}-q_{1}^{-1}\right),
\end{aligned}
$$

where the range of $i$ can be changed to $0 \leq i \leq[(t-2 p+1) / 2]$ on account of the factor $[t-2 p-2 i+1]_{1}$. Hence,

$$
\begin{aligned}
A_{p}+B_{p}= & \sum_{i=0}^{[(t-2 p+1) / 2]} \tilde{\psi}_{i} D_{(s-p-i) \alpha_{0}+(t-2 p-2 i+1) \alpha_{1}}^{-} \\
& \times\left([2 p+2 i-1]_{1}+[t-2 p-2 i+1]_{1} q_{1}^{t}\right) q^{(-2 s+t+1)(p+i)}\left(q_{1}-q_{1}^{-1}\right),
\end{aligned}
$$

which is equal to $d_{s \alpha_{0}+t \alpha_{1}, p}[t]_{1}$. We obtain (3) for $s$.

$(4)_{s}$ We use $(3)_{s}$. By Lemma 5.6 and $(3)_{s}$, we have

$$
\begin{aligned}
\Delta & \left(T_{\varpi_{1}}\left(D_{s \alpha_{0}+t \alpha_{1}}^{-}\right)\right) \\
\equiv & T_{\varpi_{1}}\left(c^{s} k_{1}^{-2 s+t}\right) \otimes T_{\varpi_{1}}\left(D_{s \alpha_{0}+t \alpha_{1}}^{-}\right) \\
& +\sum_{p=1}^{[(t+1) / 2]} T_{\varpi_{1}}\left(c^{s-p} k_{1}^{-2 s+t+1} E_{p \delta-\alpha_{1}}\right) \otimes T_{\varpi_{1}}\left(d_{s \alpha_{0}+t \alpha_{1}, p}\right) \theta(t \geq 1) \\
& +\left(q_{1}-q_{1}^{-1}\right)\left[T_{\varpi_{1}}\left(c^{s} k_{1}^{-2 s+t}\right) \otimes T_{\varpi_{1}}\left(D_{s \alpha_{0}+t \alpha_{1}}^{-}\right), c^{-1} k_{1} E_{\delta-\alpha_{1}} \otimes T_{\varpi_{1}}\left(E_{\alpha_{1}}\right)\right] \\
\equiv & c^{3 s-t} k_{1}^{-2 s+t} \otimes T_{\varpi_{1}}\left(D_{s \alpha_{0}+t \alpha_{1}}^{-}\right) \\
& +\sum_{p=2}^{[(t+3) / 2]} c^{3 s-t-p} k_{1}^{-2 s+t+1} E_{p \delta-\alpha_{1}} \otimes T_{\varpi_{1}}\left(d_{s \alpha_{0}+t \alpha_{1}, p-1}\right) \theta(t \geq 1) \\
& +\left(q_{1}-q_{1}^{-1}\right) c^{3 s-t-1} k_{1}^{-2 s+t+1} E_{\delta-\alpha_{1}} \otimes T_{\varpi_{1}}\left(\left[D_{s \alpha_{0}+t \alpha_{1}}^{-}, E_{\alpha_{1}}\right]_{q^{-2 s+t}}\right)
\end{aligned}
$$

where $\equiv$ means the congruence modulo $\mathbf{U}^{0} \mathbf{U}^{+}(<)_{\leq-2} \otimes \mathbf{U}$. Noting that both sides belong to $\mathbf{U}^{0} \mathbf{U}^{+}(<) \otimes \mathbf{U}^{+}$by Corollary 5.5 and Lemma 6.8 , we obtain (4) for $s$.

(5) for $s$ with $t=2 s-1$ We use (1) $s$. We rewrite the right hand side of (5) for $s$ with $t=2 s-1$, which is denoted by RHS. Using Lemma 7.2 (2), we 
have

$\mathrm{RHS}=T_{\varpi_{1}}\left(D_{s \alpha_{0}+(2 s-2) \alpha_{1}}^{-}\right)[2 s+2]_{1}-\sum_{l=1}^{s-1} E_{(l+1) \delta-\alpha_{1}} E_{(s-l+1) \delta-\alpha_{1}}[2 l+1]_{1} q^{-l}$.

By $(1)_{s}$ and Lemma $7.2(2)$, we have

$$
T_{\varpi_{1}}\left(D_{s \alpha_{0}+(2 s-2) \alpha_{1}}^{-}\right)[2 s-2]_{1}=\sum_{l=1}^{s-1} E_{(l+1) \delta-\alpha_{1}} E_{(s-l+1) \delta-\alpha_{1}}[2 l-1]_{1} q^{-l+1} .
$$

Hence,

$$
\mathrm{RHS}[2 s-2]_{1}=\sum_{l=1}^{s-1} E_{(l+1) \delta-\alpha_{1}} E_{(s-l+1) \delta-\alpha_{1}} c_{s, l}
$$

where we set

$$
c_{s, l}=\left([2 s+2]_{1}[2 l-1]_{1} q-[2 s-2]_{1}[2 l+1]_{1}\right) q^{-l}
$$

for $s, l \in \mathbf{Z}$. Using Lemma 3.17, for $s \in \mathbf{Z}, l \geq 1$, we have

$$
\begin{aligned}
\sum_{i=1}^{l-1} b_{2 i} c_{s, l-i} \theta(l \geq 2)= & {[2 s+2]_{1}[2 l-1]_{1}[l-1](q-1) } \\
& -[2 s-2]_{1}[2 l+1]_{1}[l](q-1)+b_{2 l}[2 s-2]_{1} .
\end{aligned}
$$

Hence, for $s \in \mathbf{Z}, l \geq 1$, we have

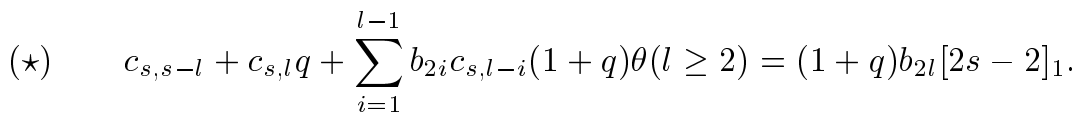

Using Lemma 3.17, we also have

$$
\sum_{i=1}^{m} b_{2 i-1} c_{2 m+1, m-i+1}=b_{2 m+1}[4 m]_{1} \quad \text { for } m \geq 1 .
$$

Now, we consider the case where $s=2 m$ with $m \geq 1$. Using Corollary 3.20 (2), we have

$$
\begin{aligned}
\operatorname{RHS}[4 m-2]_{1}= & \sum_{l=m}^{2 m-1} E_{(l+1) \delta-\alpha_{1}} E_{(2 m-l+1) \delta-\alpha_{1}} c_{2 m, l} \\
& +\sum_{l=1}^{m-1}\left(E_{(2 m-l+1) \delta-\alpha_{1}} E_{(l+1) \delta-\alpha_{1}} q\right.
\end{aligned}
$$




$$
\begin{aligned}
& +\sum_{i=1}^{m-l-1} E_{(2 m-l-i+1) \delta-\alpha_{1}} E_{(l+i+1) \delta-\alpha_{1}} b_{2 i}(1+q) \theta(l \leq m-2) \\
& \left.+E_{(m+1) \delta-\alpha_{1}}^{2} b_{2(m-l)}\right) c_{2 m, l} \theta(m \geq 2) .
\end{aligned}
$$

In the first term, putting $l=2 m-l^{\prime}$, we see that $m \leq l \leq 2 m-1$ is equivalent to $1 \leq l^{\prime} \leq m$. In the double summation, putting $l=l^{\prime}-i$, we see that $1 \leq l \leq m-2,1 \leq i \leq m-l-1$ is equivalent to $2 \leq l^{\prime} \leq m-1,1 \leq i \leq l^{\prime}-1$. Hence,

$$
\begin{aligned}
\operatorname{RHS}[4 m-2]_{1}= & \theta(m \geq 2) \sum_{l=1}^{m-1} E_{(2 m-l+1) \delta-\alpha_{1}} E_{(l+1) \delta-\alpha_{1}} \\
& \times\left(c_{2 m, 2 m-l}+c_{2 m, l} q+\sum_{i=1}^{l-1} b_{2 i} c_{2 m, l-i}(1+q) \theta(l \geq 2)\right) \\
& +E_{(m+1) \delta-\alpha_{1}}^{2}\left(c_{2 m, m}+\sum_{i=1}^{m-1} b_{2 i} c_{2 m, m-i} \theta(m \geq 2)\right)
\end{aligned}
$$

Applying ( $\star$, we have

$$
\mathrm{RHS}=(1+q) \sum_{l=1}^{m-1} b_{2 l} E_{(2 m-l+1) \delta-\alpha_{1}} E_{(l+1) \delta-\alpha_{1}}+b_{2 m} E_{(m+1) \delta-\alpha_{1}}^{2},
$$

which is equal to $\left[E_{\delta-\alpha_{1}}, E_{(2 m+1) \delta-\alpha_{1}}\right]_{q}$ by Corollary $3.20(2)$. Next, we consider the case where $s=2 m+1$ with $m \geq 1$. Using Corollary 3.20 (2), we have

$$
\begin{aligned}
\operatorname{RHS}[4 m]_{1}= & \sum_{l=m+1}^{2 m} E_{(l+1) \delta-\alpha_{1}} E_{(2 m-l+2) \delta-\alpha_{1}} c_{2 m+1, l} \\
& +\sum_{l=1}^{m}\left(E_{(2 m-l+2) \delta-\alpha_{1}} E_{(l+1) \delta-\alpha_{1}} q\right. \\
& +\sum_{i=1}^{m-l} E_{(2 m-l-i+2) \delta-\alpha_{1}} E_{(l+i+1) \delta-\alpha_{1}} b_{2 i}(1+q) \theta(l \leq m-1) \\
& \left.+E_{(2 m+2) \delta-\alpha_{0}} b_{2(m-l)+1}[4]_{1}\right) c_{2 m+1, l} .
\end{aligned}
$$

In the first term, putting $l=2 m-l^{\prime}+1$, we see that $m+1 \leq l \leq 2 m$ is equivalent to $1 \leq l^{\prime} \leq m$. In the double summation, putting $l=l^{\prime}-i$, we see 
that $1 \leq l \leq m-1,1 \leq i \leq m-l$ is equivalent to $2 \leq l^{\prime} \leq m, 1 \leq l^{\prime}-1$. Hence,

$$
\begin{aligned}
\operatorname{RHS}[4 m]_{1}= & \sum_{l=1}^{m} E_{(2 m-l+2) \delta-\alpha_{1}} E_{(l+1) \delta-\alpha_{1}} \\
& \times\left(c_{2 m+1,2 m-l+1}+c_{2 m+1, l} q+\sum_{i=1}^{l-1} b_{2 i} c_{2 m+1, l-i}(1+q) \theta(l \geq 2)\right) \\
& +E_{(2 m+2) \delta-\alpha_{0}} \sum_{i=1}^{m} b_{2 i-1} c_{2 m+1, m-i+1}[4]_{1} .
\end{aligned}
$$

Applying $(\star)$ and $(\star \star)$, we have

$$
\mathrm{RHS}=(1+q) \sum_{l=1}^{m} b_{2 l} E_{(2 m-l+2) \delta-\alpha_{1}} E_{(l+1) \delta-\alpha_{1}}+[4]_{1} b_{2 m+1} E_{(2 m+2) \delta+\alpha_{0}},
$$

which is equal to $\left[E_{\delta-\alpha_{1}}, E_{(2 m+2) \delta-\alpha_{1}}\right]_{q}$ by Corollary 3.20 (2). Thus, we obtain (5) for $s$ with $t=2 s-1$.

$(5)_{s}$ We use $(2)_{s}$ and $(4)_{s}$. The case where $t=2 s-1$ is proved above. The case where $t=0$ is clear from Lemma 3.9. We assume that $s \geq 2,1 \leq t \leq 2 s-2$ and argue by the descending induction on $t$. The left and right hand sides of (5) (for $s$ and $t$ ) are denoted by LHS and RHS respectively. By Corollary 4.7, both of LHS and RHS belong to $\mathbf{U}^{+}\left(<; \delta-\alpha_{1}\right)$; thus, we can use Corollary 5.10 to prove (5) for $s$. Using Lemma 5.3 with $n=1$ and $(4)_{s}$, we have

$$
\begin{aligned}
\Delta(\mathrm{LHS})= & {\left[\Delta\left(E_{\delta-\alpha_{1}}\right), \Delta\left(T_{\varpi_{1}}\left(D_{s \alpha_{0}+t \alpha_{1}}^{-}\right)\right)\right]_{q^{2 s-t}} } \\
\equiv & {\left[c k_{1}^{-1} \otimes E_{\delta-\alpha_{1}}+E_{\delta-\alpha_{1}} \otimes 1, c^{3 s-t} k_{1}^{-2 s+t} \otimes T_{\varpi_{1}}\left(D_{s \alpha_{0}+t \alpha_{1}}^{-}\right)\right.} \\
& +\sum_{p=2}^{[(t+3) / 2]} c^{3 s-t-p} k_{1}^{-2 s+t+1} E_{p \delta-\alpha_{1}} \otimes T_{\varpi_{1}}\left(d_{s \alpha_{0}+t \alpha_{1}, p-1}\right) \theta(t \geq 1) \\
& +c^{3 s-t-1} k_{1}^{-2 s+t+1} E_{\delta-\alpha_{1}} \\
& \left.\otimes T_{\varpi_{1}}\left(\left[D_{s \alpha_{0}+t \alpha_{1}}^{-}, E_{\alpha_{1}}\right]_{q^{-2 s+t}}\right)\left(q_{1}-q_{1}^{-1}\right)\right] \\
\equiv & c^{3 s-t+1} k_{1}^{-2 s+t-1} \otimes \mathrm{LHS}+\sum_{p=1}^{[(t+3) / 2]} c^{3 s-t-p+1} k_{1}^{-2 s+t} E_{p \delta-\alpha_{1}} \otimes A_{p}
\end{aligned}
$$

where we set

$$
A_{p}=\left[E_{\delta-\alpha_{1}}, T_{\varpi_{1}}\left(d_{s \alpha_{0}+t \alpha_{1}, p-1}\right)\right]_{q^{2 s-t-1}}
$$


for $2 \leq p \leq[(t+3) / 2]$, and

$$
\begin{aligned}
A_{1}= & {\left[E_{\delta-\alpha_{1}}, T_{\varpi_{1}}\left(\left[D_{s \alpha_{0}+t \alpha_{1}}^{-}, E_{\alpha_{1}}\right]_{q^{-2 s+t}}\right)\right]_{q^{2 s-t-1}}\left(q_{1}-q_{1}^{-1}\right) } \\
& +T_{\varpi_{1}}\left(D_{s \alpha_{0}+t \alpha_{1}}^{-}\right)\left(q^{-2 s+t}-q^{2 s-t}\right) .
\end{aligned}
$$

Note that if $t$ is odd and $p=(t+3) / 2$, then $A_{p}=0$, so that we have $A_{p}$ for $1 \leq p \leq[(t+2) / 2]$. Next, we calculate the coproduct of RHS. By $(4)_{s}$, we have

$$
\begin{aligned}
\Delta(\mathrm{RHS})= & \Delta\left(T_{\varpi_{1}}\left(D_{s \alpha_{0}+(t-1) \alpha_{1}}^{-}\right)[4 s-t+1]_{1}\right. \\
& \left.-\sum_{i=1}^{[t / 2]} E_{(i+1) \delta-\alpha_{i}} T_{\varpi_{1}}\left(D_{(s-i) \alpha_{0}+(t-2 i) \alpha_{1}}^{-}\right)[2 i+1]_{1} q^{(-2 s+t) i} \theta(t \geq 2)\right) .
\end{aligned}
$$

For $t \geq 2,1 \leq i \leq[t / 2]$, using Lemma 5.3 and $(4)_{s-1}$, we have

$$
\begin{aligned}
\Delta & \left(E_{(i+1) \delta-\alpha_{1}} T_{\varpi_{1}}\left(D_{(s-i) \alpha_{0}+(t-2 i) \alpha_{1}}^{-}\right)\right) \\
\equiv & \left(c^{i+1} k_{1}^{-1} \otimes E_{(i+1) \delta-\alpha_{1}}+\left(q_{1}-q_{1}^{-1}\right) \sum_{p=1}^{i+1} c^{i-p+1} E_{p \delta-\alpha_{1}} \otimes \tilde{\psi}_{i-p+1}\right) \\
& \times\left(c^{3 s-t-i} k_{1}^{-2 s+t} \otimes T_{\varpi_{1}}\left(D_{(s-i) \alpha_{0}+(t-2 i) \alpha_{1}}^{-}\right)\right. \\
& +\sum_{p=2}^{[(t-2 i+3) / 2]} c^{3 s-t-i-p} k_{1}^{-2 s+t+1} E_{p \delta-\alpha_{1}} \\
& \otimes T_{\varpi_{1}}\left(d_{\left.(s-i) \alpha_{0}+(t-2 i) \alpha_{1}, p-1\right)}\right)(t-2 i \geq 1) \\
& \left.+c^{3 s-t-i-1} k_{1}^{-2 s+t+1} E_{\delta-\alpha_{1}} \otimes T_{\varpi_{1}}\left(\left[D_{(s-i) \alpha_{0}+(t-2 i) \alpha_{1}}^{-}, E_{\alpha_{1}}\right]_{q^{-2 s+t}}\right)\left(q_{1}-q_{1}^{-1}\right)\right) \\
\equiv & c^{3 s-t+1} k_{1}^{-2 s+t-1} \otimes E_{(i+1) \delta-\alpha_{1}} T_{\varpi_{1}}\left(D_{(s-i) \alpha_{0}+(t-2 i) \alpha_{1}}^{-}\right) \\
& +\sum_{p=2}^{[(t-2 i+3) / 2]} c^{3 s-t-p+1} k_{1}^{-2 s+t} E_{p \delta-\alpha_{1}} \otimes E_{(i+1) \delta-\alpha_{1}} T_{\varpi_{1}}\left(d_{(s-i) \alpha_{0}+(t-2 i) \alpha_{1}, p-1}\right) \\
& +E_{\delta-\alpha_{1}} c^{3 s-t} k_{1}^{-2 s+t} \\
& \otimes E_{(i+1) \delta-\alpha_{1}} T_{\varpi_{1}}\left(\left[D_{(s-i) \alpha_{0}+(t-2 i) \alpha_{1}}^{-}, E_{\alpha_{1}}\right]_{q^{-2 s+t}}\right)\left(q_{1}-q_{1}^{-1}\right) q^{2 s-t} \\
& +\sum_{p=1}^{i+1} k_{1}^{-2 s+t} E_{p \delta-\alpha_{1}} \otimes \tilde{\psi}_{i-p+1} T_{\varpi_{1}}\left(D_{(s-i) \alpha_{0}+(t-2 i) \alpha_{1}}^{-}\right) q^{-2 s+t}\left(q_{1}-q_{1}^{-1}\right) .
\end{aligned}
$$

We sum up this for $1 \leq i \leq[t / 2]$ after multiplying $[2 i+1]_{1} q^{(-2 s+t) i}$. Note that $1 \leq i \leq[t / 2], 2 \leq p \leq[(t-2 i+3) / 2]$ is equivalent to $2 \leq p \leq[(t+1) / 2], 1 \leq$ 
$i \leq[(t-2 p+3) / 2]$ and that $1 \leq i \leq[t / 2], 1 \leq p \leq i+1$ is equivalent to $1 \leq p \leq[(t+2) / 2], \max (1, p-1) \leq i \leq[t / 2]$. Thus, for $t \geq 2$, we have

$$
\begin{aligned}
& \sum_{i=1}^{[t / 2]} \Delta\left(E_{(i+1) \delta-\alpha_{1}} T_{\varpi_{1}}\left(D_{(s-i) \alpha_{0}+(t-2 i) \alpha_{1}}^{-}\right)\right)[2 i+1]_{1} q^{(-2 s+t) i} \\
& \equiv c^{3 s-t+1} k_{1}^{-2 s+t-1} \otimes \sum_{i=1}^{[t / 2]} E_{(i+1) \delta-\alpha_{1}} T_{\varpi_{1}}\left(D_{(s-i) \alpha_{0}+(t-2 i) \alpha_{1}}^{-}\right)[2 i+1]_{1} q^{(-2 s+t) i} \\
& \quad+\sum_{p=2}^{[(t+1) / 2]} c^{3 s-t-p+1} k_{1}^{-2 s+t} E_{p \delta-\alpha_{1}} \\
& \quad \otimes \sum_{i=1}^{[(t-2 p+3) / 2]} E_{(i+1) \delta-\alpha_{1}} T_{\varpi_{1}}\left(d_{(s-i) \alpha_{0}+(t-2 i) \alpha_{1}, p-1}\right)[2 i+1]_{1} q^{(-2 s+t) i} \\
& \quad+E_{\delta-\alpha_{1}} c^{3 s-t} k_{1}^{-2 s+t} \otimes \sum_{i=1}^{[t / 2]} E_{(i+1) \delta-\alpha_{1}} T_{\varpi_{1}}\left(\left[D_{(s-i) \alpha_{0}+(t-2 i) \alpha_{1}}^{-}, E_{\alpha_{1}}\right]_{q^{-2 s+t}}\right) \\
& \quad \times[2 i+1]_{1} q^{(-2 s+t)(i-1)}\left(q_{1}-q_{1}^{-1}\right) \\
& \quad+\sum_{p=1}^{[(t+2) / 2]} k_{1}^{-2 s+t} E_{p \delta-\alpha_{1}} \otimes \sum_{i=\max _{(1, p-1)}}^{[t / 2]} \tilde{\psi}_{i-p+1} T_{\varpi_{1}}\left(D_{(s-i) \alpha_{0}+(t-2 i) \alpha_{1}}^{-}\right) \\
& \quad \times[2 i+1]_{1} q^{(-2 s+t)(i+1)}\left(q_{1}-q_{1}^{-1}\right) .
\end{aligned}
$$

Hence,

$$
\Delta(\mathrm{RHS}) \equiv c^{3 s-t+1} k_{1}^{-2 s+t-1} \otimes \mathrm{RHS}+\sum_{p=1}^{[(t+2) / 2]} c^{3 s-t-p+1} k_{1}^{-2 s+t} E_{p \delta-\alpha_{1}} \otimes B_{p}
$$

where we set

$$
\begin{aligned}
B_{p}= & T_{\varpi_{1}}\left(d_{s \alpha_{0}+(t-1) \alpha_{1}, p-1}\right)[4 s-t+1]_{1} \\
& -\sum_{i=1}^{[(t-2 p+3) / 2]} E_{(i+1) \delta-\alpha_{1}} T_{\varpi_{1}}\left(d_{(s-i) \alpha_{0}+(t-2 i) \alpha_{1}, p-1}\right) \\
& \times[2 i+1]_{1} q^{(-2 s+t) i} \theta(p \leq[(t+1) / 2]) \\
& -\sum_{i=p-1}^{[t / 2]} \tilde{\psi}_{i-p+1} T_{\varpi_{1}}\left(D_{(s-i) \alpha_{0}+(t-2 i) \alpha_{1}}^{-}\right)[2 i+1]_{1} q^{(-2 s+t)(i+1)}\left(q_{1}-q_{1}^{-1}\right)
\end{aligned}
$$

for $t \geq 2,2 \leq p \leq[(t+2) / 2]$ and

$$
B_{1}=T_{\varpi_{1}}\left(\left[D_{s \alpha_{0}+(t-1) \alpha_{1}}^{-}, E_{\alpha_{1}}\right]_{q^{-2 s+t-1}}\right)[4 s-t+1]_{1}\left(q_{1}-q_{1}^{-1}\right)
$$




$$
\begin{aligned}
& -\sum_{i=1}^{[t / 2]} E_{(i+1) \delta-\alpha_{1}} T_{\varpi_{1}}\left(\left[D_{(s-i) \alpha_{0}+(t-2 i) \alpha_{1}}^{-}, E_{\alpha_{1}}\right]_{q^{-2 s+t-1}}\right) \\
& \times[2 i+1]_{1}\left(q_{1}-q_{1}^{-1}\right) q^{(-2 s+t) i} \theta(t \geq 2) \\
& -\sum_{i=1}^{[t / 2]} \tilde{\psi}_{i} T_{\varpi_{1}}\left(D_{(s-i) \alpha_{0}+(t-2 i) \alpha_{1}}^{-}\right)[2 i+1]_{1} q^{(-2 s+t)(i+1)}\left(q_{1}-q_{1}^{-1}\right) \theta(t \geq 2) .
\end{aligned}
$$

By virtue of Corollary 5.10, the proof of (5) for $s$ and $t$ is reduced to showing that $A_{p}=B_{p}$ for $1 \leq p \leq[(t+2) / 2]$. First, assuming that $t \geq 2$, we shall check that $A_{p}=B_{p}$ for $2 \leq p \leq[(t+2) / 2]$. We rewrite $A_{p}$. Using Proposition 3.26 (5), we have

$$
\begin{aligned}
A_{p}= & {\left[E_{\delta-\alpha_{1}}, T_{\varpi_{1}}\left(d_{s \alpha_{0}+t \alpha_{1}, p-1}\right)\right]_{q^{2 s-t-1}} } \\
= & {\left[E_{\delta-\alpha_{1}}, \sum_{i=0}^{[(t-2 p+3) / 2]} \tilde{\psi}_{i} T_{\varpi_{1}}\left(D_{(s-p-i) \alpha_{0}+(t-2 p-2 i+3) \alpha_{1}}^{-}\right)\right]_{q^{2 s-t-1}} } \\
& \times q_{1}^{(-2 s+t)(2 p+2 i-2)+t+1}\left(q_{1}-q_{1}^{-1}\right) \\
= & \sum_{i=0}^{[(t-2 p+3) / 2]}\left(\tilde{\psi}_{i}\left[E_{\delta-\alpha_{1}}, T_{\varpi_{1}}\left(D_{(s-p-i+1) \alpha_{0}+(t-2 p-2 i+3) \alpha_{1}}^{-}\right)\right]_{q^{2 s-t-1}}\right. \\
& \left.+\left(1+q^{-1}\right) \sum_{j=0}^{i-1} b_{2(i-j)} \tilde{\psi}_{j} E_{(i-j+1) \delta-\alpha_{1}} T_{\varpi_{1}}\left(D_{(s-p-i) \alpha_{0}+(t-2 p-2 i+3) \alpha_{1}}^{-}\right)\right) \\
& \times q_{1}^{(-2 s+t)(2 p+2 i-2)+t+1}\left(q_{1}-q_{1}^{-1}\right) \theta(p \leq[(t+1) / 2]) .
\end{aligned}
$$

We apply $(5)_{s-1}$ to the first term. In the second one, putting $j=i^{\prime}, i-j=j^{\prime}$, we see that $i=i^{\prime}+j^{\prime}$ and that $1 \leq i \leq[(t-2 p+3) / 2], 0 \leq j \leq i-1$ is equivalent to $0 \leq i^{\prime} \leq[(t-2 p+1) / 2], 1 \leq j \leq[(t-2 p-2 i+3) / 2]$. Hence,

$$
\begin{aligned}
A_{p}= & \sum_{i=0}^{[(t-2 p+3) / 2]} \tilde{\psi}_{i}\left(T_{\varpi_{1}}\left(D_{(s-p-i+1) \alpha_{0}+(t-2 p-2 i+2) \alpha_{1}}^{-}\right)\right. \\
& \times[4 s-t-2 p-2 i+2]_{1} \theta(t-2 p-2 i+2 \geq 0) \\
& -\sum_{j=1}^{[(t-2 p-2 i+3) / 2]} E_{(j+1) \delta-\alpha_{i}} T_{\varpi_{1}}\left(D_{(s-p-i-j+1) \alpha_{0}+(t-2 p-2 i-2 j+3) \alpha_{1}}^{-}\right)[2 j+1]_{1} \\
& \left.\times q^{(-2 s+t+1) j} \theta(t-2 p-2 i+1 \geq 0)\right) q_{1}^{(-2 s+t)(2 p+2 i-2)+t+1}\left(q_{1}-q_{1}^{-1}\right) \\
& +\sum_{i=0}^{[(t-2 p+1) / 2][(t-2 p-2 i+3) / 2]} \tilde{\psi}_{i=1} E_{(j+1) \delta-\alpha_{1}}
\end{aligned}
$$




$$
\begin{aligned}
& \times T_{\varpi_{1}}\left(D_{(s-p-i-j+1) \alpha_{0}+(t-2 p-2 i-2 j+3) \alpha_{1}}^{-}\right) \\
& \times b_{2 j} q_{1}^{(-2 s+t)(2 p+2 i+2 j-1)+2 s+1}\left(1+q^{-1}\right)\left(q_{1}-q_{1}^{-1}\right) \theta(p \leq[(t+1) / 2]) \\
= & \sum_{i=0}^{[(t-2 p+2) / 2]} \tilde{\psi}_{i} T_{\varpi_{1}}\left(D_{(s-p-i+1) \alpha_{0}+(t-2 p-2 i+2) \alpha_{1}}^{-}\right)[4 s-t-2 p-2 i+2]_{1} \\
& \times q_{1}^{(-2 s+t)(2 p+2 i-1)+2 s+1}\left(q_{1}-q_{1}^{-1}\right) \\
& +\sum_{i=0} \sum_{j=1}^{(t-2 p+1) / 2][(t-2 p-2 i+3) / 2]} \tilde{\psi}_{i} E_{(j+1) \delta-\alpha_{1}} \\
& \times T_{\varpi_{1}}\left(D_{(s-p-i-j+1) \alpha_{0}+(t-2 p-2 i-2 j+3) \alpha_{1}}^{-}\right) \\
& \times\left(\left(1+q^{-1}\right) b_{2 j}-[2 j+1]_{1} q^{j}\right) q_{1}^{(-2 s+t)(2 p+2 i+2 j-1)+2 s+1} \\
& \times\left(q_{1}-q_{1}^{-1}\right) \theta(p \leq[(t+1) / 2]) .
\end{aligned}
$$

We rewrite $B_{p}$. Assuming that $p \leq[(t+1) / 2]$ and using Proposition $3.26(5)$, we have

$$
\begin{aligned}
\sum_{i=1}^{[(t-2 p+3) / 2]} E_{(i+1) \delta-\alpha_{1}} T_{\varpi_{1}}\left(d_{(s-i) \alpha_{0}+(t-2 i) \alpha_{1}, p-1}\right) \\
\times[2 i+1]_{1} q^{(-2 s+t) i} \theta(p \leq[(t+1) / 2]) \\
=\sum_{i=1}^{[(t-2 p+3) / 2][(t-2 p-2 i+3) / 2]} \sum_{j=0} E_{(i+1) \delta-\alpha_{1}} \\
\quad \times \tilde{\psi}_{j} T_{\varpi_{1}}\left(D_{(s-p-i-j+1) \alpha_{0}+(t-2 p-2 i-2 j+3) \alpha_{1}}^{-}\right) \\
\quad \times q_{1}^{(-2 s+t)(2 p+2 j-1)+2 s-2 i+1}\left(q_{1}-q_{1}^{-1}\right) \\
=\quad \sum_{i=1}^{[(t-2 p+3) / 2][(t-2 p-2 i+3) / 2]}\left(\tilde{\psi}_{j} E_{(i+1) \delta-\alpha_{1}}\right. \\
\left.\quad+\left(1+q^{-1}\right) \sum_{k=0}^{j-1} b_{2(j-k)} \tilde{\psi}_{k} E_{(i+j-k+1) \delta-\alpha_{1}} \theta(j \geq 1)\right) \\
\quad \times T_{\varpi_{1}}\left(D_{(s-p-i-j+1) \alpha_{0}+(t-2 p-2 i-2 j+3) \alpha_{1}}^{-}\right) \\
\quad \times q_{1}^{(-2 s+t)(2 p+2 j-1)+2 s-2 i+1}\left(q_{1}-q_{1}^{-1}\right) .
\end{aligned}
$$

In the double summation, we see that $1 \leq i \leq[(t-2 p+3) / 2], 0 \leq j \leq[(t-2 p-$ $2 i+3) / 2]$ is equivalent to $0 \leq j \leq[(t-2 p+1) / 2], 1 \leq i \leq[(t-2 p-2 j+3) / 2]$. In the triple one, putting $k=i^{\prime}, i+j-k=j^{\prime}, j-k=k^{\prime}$, we see that $i=j^{\prime}-k^{\prime}$, $j=i^{\prime}+k^{\prime}$ and that $1 \leq i \leq[(t-2 p+3) / 2], 1 \leq j \leq[(t-2 p-2 i+3) / 2], 0 \leq$ 
$k \leq j-1$ is equivalent to $0 \leq i^{\prime} \leq[(t-2 p+1) / 2], 2 \leq j^{\prime} \leq\left[\left(t-2 p-2 i^{\prime}+3\right) / 2\right]$, $0 \leq k^{\prime} \leq j^{\prime}-1$. Hence,

$$
\begin{aligned}
B_{p}= & \sum_{i=0}^{[(t-2 p+2) / 2]} \tilde{\psi}_{i} T_{\varpi_{1}}\left(D_{(s-p-i+1) \alpha_{0}+(t-2 p-2 i+2) \alpha_{1}}^{-}\right) c_{i}\left(q_{1}-q_{1}^{-1}\right) \\
& -\sum_{i=0}^{[(t-2 p+1) / 2]} \sum_{j=1}^{[(t-2 p-2 i+3) / 2]} \tilde{\psi}_{i} E_{(j+1) \delta-\alpha_{1}} \\
& \times T_{\varpi_{1}}\left(D_{(s-p-i-j+1) \alpha_{0}+(t-2 p-2 i-2 j+2) \alpha_{1}}^{-}\right) \\
& \times c_{i j}\left(q_{1}-q_{1}^{-1}\right) \theta(p \leq[(t+1) / 2])
\end{aligned}
$$

where we set

$$
c_{i}=[4 s-t+1]_{1} q_{1}^{(-2 s+t)(2 p+2 i-1)+2 s-2 p-2 i+2}-[2 p+2 i-1]_{1} q^{(-2 s+t)(p+i)}
$$

and

$$
\begin{aligned}
c_{i j}= & {[2 j+1]_{1} q_{1}^{(-2 s+t)(2 p+2 i+2 j-1)+2 s-2 p-2 j+1} } \\
& +\left(1+q^{-1}\right) \sum_{k=0}^{j-1} b_{2 k}[2 j-2 k+1]_{1} q_{1}^{(-2 s+t)(2 p+2 i+2 j-1)+2 s-2 j+2 k+1} \theta(j \geq 1) .
\end{aligned}
$$

It directly follows that

$$
c_{i}=[4 s-t-2 p-2 i+2]_{1} q_{1}^{(-2 s+t)(2 p+2 i-1)+2 s+1} .
$$

Using Lemma 3.17 (1), we have

$$
c_{i j}=\left([2 j+1]_{1} q^{j}-b_{2 j}\left(1+q^{-1}\right)\right) q_{1}^{(-2 s+t)(2 p+2 i+2 j-1)+2 s+1} .
$$

Hence we have $A_{p}=B_{p}$ for $2 \leq p \leq[(t+2) / 2]$. It remains to show that $A_{1}=B_{1}$. We rewrite $A_{1}$. Using $(2)_{s}$, Lemma 2.11, and Proposition 3.26 (5), we have

$$
\begin{aligned}
& {\left[E_{\delta-\alpha_{1}}, T_{\varpi_{1}}\left(\left[D_{s \alpha_{0}+t \alpha_{1}}^{-}, E_{\alpha_{1}}\right]_{q^{-2 s+t}}\right)\right]_{q^{2 s-t-1}}} \\
& =\left[E_{\delta-\alpha_{1}}, T_{\varpi_{1}}\left(D_{s \alpha_{0}+(t+1) \alpha_{1}}^{-}\right)[t+1]_{1}\right. \\
& \left.\quad+\sum_{i=1}^{[(t+1) / 2]} \tilde{\psi}_{i} T_{\varpi_{1}}\left(D_{(s-i) \alpha_{0}+(t-2 i+1) \alpha_{1}}^{-}\right) q_{1}^{(-2 s+t)(2 i+1)+2 s+1}\right]_{q^{2 s-t-1}} \\
& \quad=\left[E_{\delta-\alpha_{1}}, T_{\varpi_{1}}\left(D_{s \alpha_{0}+(t+1) \alpha_{1}}^{-}\right)\right]_{q^{2 s-t-1}}[t+1]_{1}
\end{aligned}
$$




$$
\begin{aligned}
& +\sum_{i=1}^{[(t+1) / 2]} \tilde{\psi}_{i}\left[E_{\delta-\alpha_{1}}, T_{\varpi_{1}}\left(D_{(s-i) \alpha_{0}+(t-2 i+1) \alpha_{1}}^{-}\right)\right]_{q^{2 s-t-1}} q_{1}^{(-2 s+t)(2 i+1)+2 s+1} \\
& +\sum_{i=1}^{[(t+1) / 2]} \sum_{j=0}^{i-1} \tilde{\psi}_{j} E_{(i-j+1) \delta-\alpha_{1}} T_{\varpi 1}\left(D_{(s-i) \alpha_{0}+(t-2 i+1) \alpha_{1}}^{-}\right) \\
& \times b_{2(i-j)} q_{1}^{(-2 s+t)(2 i+1)+2 s+1}\left(1+q^{-1}\right) .
\end{aligned}
$$

We apply the induction hypothesis $((5)$ for $s$ and $t+1)$ to the first term and $(5)_{s-1}$ to the second one. In the third one, putting $j=i^{\prime}, i-j=j^{\prime}$, we see that $i=i^{\prime}+j^{\prime}$ and that $1 \leq i \leq[(t+1) / 2], 0 \leq j \leq i-1$ is equivalent to $0 \leq i^{\prime} \leq[(t-1) / 2], 1 \leq j^{\prime} \leq\left[\left(t-2 i^{\prime}+1\right) / 2\right]$. Hence,

$$
\begin{aligned}
& {\left[E_{\delta-\alpha_{1}}, T_{\varpi_{1}}\left(\left[D_{s \alpha_{0}+t \alpha_{1}}^{-}, E_{\alpha_{1}}\right]_{q^{-2 s+t}}\right)\right]_{q^{2 s-t-1}}} \\
& =T_{\varpi_{1}}\left(D_{s \alpha_{0}+t \alpha_{1}}^{-}\right)[4 s-t]_{1}[t+1]_{1} \\
& -\sum_{i=1}^{[(t+1) / 2]} E_{(i+1) \delta-\alpha_{1}} T_{\varpi_{1}}\left(D_{(s-i) \alpha_{0}+(t-2 i+1) \alpha_{1}}^{-}\right)[2 i+1]_{1} q^{(-2 s+t+1) i}[t+1]_{1} \\
& +\sum_{i=1}^{[(t+1) / 2]} \tilde{\psi}_{i}\left(T_{\varpi_{1}}\left(D_{(s-i) \alpha_{0}+(t-2 i) \alpha_{1}}^{-}\right)[4 s-t-2 i]_{1} \theta(t-2 i \geq 0)\right. \\
& {[(t-2 i+1) / 2]} \\
& -\quad \sum_{j=1} E_{(j+1) \delta-\alpha_{1}} T_{\varpi_{1}}\left(D_{(s-i-j) \alpha_{0}+(t-2 i-2 j+1) \alpha_{1}}^{-}\right) \\
& \left.\times[2 j+1]_{1} q^{(-2 s+t+1) j} \theta(t-2 i \geq 1)\right) q_{1}^{(-2 s+t)(2 i+1)+2 s+1} \\
& +\sum_{i=0}^{[(t-1) / 2]} \sum_{j=1}^{[(t-2 i+1) / 2]} \tilde{\psi}_{i} E_{(j+1) \delta-\alpha_{1}} T_{\varpi_{1}}\left(D_{(s-i-j) \alpha_{0}+(t-2 i-2 j+1) \alpha_{1}}^{-}\right) \\
& \times b_{2 j} q_{1}^{(-2 s+t)(2 i+2 j+1)+2 s+1}\left(1+q^{-1}\right) .
\end{aligned}
$$

Thus,

$$
\begin{aligned}
A_{1}= & T_{\varpi_{1}}\left(D_{s \alpha_{0}+t \alpha_{1}}^{-}\right)\left([4 s-t]_{1}[t+1]_{1}\left(q_{1}-q_{1}^{-1}\right)+\left(q^{-2 s+t}-q^{2 s-t}\right)\right) \\
& +\sum_{i=1}^{[t / 2]} \tilde{\psi}_{i} T_{\varpi_{1}}\left(D_{(s-i) \alpha_{0}+(t-2 i) \alpha_{1}}^{-}\right)[4 s-t-2 i]_{1} q_{1}^{(-2 s+t)(2 i+1)+2 s+1}\left(q_{1}-q_{1}^{-1}\right) \\
& -\sum_{j=1}^{[(t+1) / 2]} E_{(j+1) \delta-\alpha_{1}} T_{\varpi_{1}}\left(D_{(s-j) \alpha_{0}+(t-2 j+1) \alpha_{1}}^{-}\right) \\
& \times[2 j+1]_{1}[t+1]_{1} q^{(-2 s+t+1) j}\left(q_{1}-q_{1}^{-1}\right)
\end{aligned}
$$




$$
\begin{aligned}
& -\sum_{i=0}^{[(t-1) / 2]} \sum_{j=1}^{[(t-2 i+1) / 2]} \tilde{\psi}_{i} E_{(j+1) \delta-\alpha_{1}} T_{\varpi_{1}}\left(D_{(s-i-j) \alpha_{0}+(t-2 i-2 j+1) \alpha_{1}}^{-}\right) \\
\times & {[2 j+1]_{1}[t+1]_{1} q^{(-2 s+t+1) j} q_{1}^{(-2 s+t)(2 i+1)+2 s+1}\left(q_{1}-q_{1}^{-1}\right) } \\
& -\sum_{i=0}^{[(t-1) / 2][(t-2 i+1) / 2]} \sum_{j=1}^{(-2 s+t+1) j} \tilde{\psi}_{i}^{(-2 s+t)(2 i+2 j+1)+2 s+1}\left(1+E_{(j+1) \delta-\alpha_{1}} T_{\varpi_{1}}\left(D_{(s-i-j) \alpha_{0}+(t-2 i-2 j+1) \alpha_{1}}^{-}\right)\left(q_{1}-q_{1}^{-1}\right)\right. \\
& \times b_{2 j} q^{(-2 s)} \\
= & T_{\varpi_{1}}\left(D_{s \alpha_{0}+t \alpha_{1}}^{-}\right)[4 s-t+1]_{1}[t]_{1}\left(q_{1}-q_{1}^{-1}\right) \\
+ & \sum_{i=1}^{[t / 2]} \tilde{\psi}_{i} T_{\varpi_{1}}\left(D_{(s-i) \alpha_{0}+(t-2 i) \alpha_{1}}^{-}\right)[4 s-t-2 i]_{1} \\
\times & q_{1}^{(-2 s+t)(2 i+1)+2 s+1}\left(q_{1}-q_{1}^{-1}\right) \theta(t \geq 2) \\
+ & \sum_{j=1}^{[(t+1) / 2]} E_{(j+1) \delta-\alpha_{1}} T_{\varpi_{1}}\left(D_{(s-j) \alpha_{0}+(t-2 j+1) \alpha_{1}}^{-}\right) \\
\times & \left(\left(1+q^{-1}\right) b_{2 j} q_{1}^{(-2 s+t)(2 j+1)+2 s+1}\right. \\
- & {\left.[2 j+1]_{1}[t+1]_{1} q^{(-2 s+t+1) j}\left(q_{1}-q_{1}^{-1}\right)\right) } \\
& {[(t-1) / 2][(t-2 i+1) / 2] } \\
+ & \sum_{i=1} \sum_{j=1} \tilde{\psi}_{i} E_{(j+1) \delta-\alpha_{1}} T_{\varpi_{1}}\left(D_{(s-i-j) \alpha_{0}+(t-2 i-2 j+1) \alpha_{1}}^{-}\right) \\
\times & \left(\left(1+q^{-1}\right) b_{2 j}-[2 j+1]_{1} q^{j}\right) q_{1}^{(-2 s+t)(2 i+2 j+1)+2 s+1}\left(q_{1}-q_{1}^{-1}\right) \theta(t \geq 3) .
\end{aligned}
$$

We rewrite $B_{1}$. Using $(2)_{s-1}$ and Proposition 3.26 (5), we have

$$
\begin{aligned}
& \sum_{i=1}^{[t / 2]} E_{(i+1) \delta-\alpha_{1}} T_{\varpi_{1}}\left(\left[D_{(s-i) \alpha_{0}+(t-2 i) \alpha_{1}}^{-}, E_{\alpha_{1}}\right]_{q^{-2 s+t-1}}\right)[2 i+1]_{1} q^{(-2 s+t) i} \theta(t \geq 2) \\
& =\sum_{i=1}^{[t / 2]} E_{(i+1) \delta-\alpha_{1}}\left(T_{\varpi_{1}}\left(D_{(s-i) \alpha_{0}+(t-2 i+1) \alpha_{1}}^{-}\right)[t-2 i+1]_{1}\right. \\
& \quad+\sum_{i=1}^{[(t-2 i+1) / 2]} \tilde{\psi}_{i} T_{\varpi_{1}}\left(D_{(s-i-j) \alpha_{0}+(t-2 i-2 j+1) \alpha_{1}}^{-}\right) \\
& \left.\quad \times q_{1}^{(-2 s+t)(2 j+1)+2(s-i)+1} \theta(t-2 i \geq 1)\right)[2 i+1]_{1} q^{(-2 s+t) i} \theta(t \geq 2) \\
& =\sum_{i=1}^{[t / 2]} E_{(i+1) \delta-\alpha_{1}} T_{\varpi_{1}}\left(D_{(s-i) \alpha_{0}+(t-2 i+1) \alpha_{1}}^{-}\right)[t-2 i+1]_{1}[2 i+1]_{1} q^{(-2 s+t) i} \theta(t \geq 2)
\end{aligned}
$$




$$
\begin{aligned}
& +\sum_{i=1}^{[t / 2]} \sum_{j=1}^{[(t-2 i+1) / 2]}\left(\tilde{\psi}_{j} E_{(i+1) \delta-\alpha_{1}}+\left(1+q^{-1}\right) \sum_{k=0}^{j-1} b_{2(j-k)} \tilde{\psi}_{k} E_{(i+j-k+1) \delta-\alpha_{1}}\right) \\
& \times T_{\varpi_{1}}\left(D_{(s-i-j) \alpha_{0}+(t-2 i-2 j+1) \alpha_{1}}^{-}\right)[2 i+1]_{1} \\
& \times q_{1}^{(-2 s+t)(2 i+2 j+1)+2(s-i)+1} \theta(t-2 i \geq 1) \theta(t \geq 3) .
\end{aligned}
$$

In the first term, on account of the factor $[t-2 i+1]_{1}$, the range of $i$ can be changed to $1 \leq i \leq[(t+1) / 2]$, so that the case where $t=1$ can be included. In the double summation, we see that $1 \leq i \leq[(t-1) / 2], 1 \leq j \leq[(t-2 i+1) / 2]$ is equivalent to $1 \leq j \leq[(t-1) / 2], 1 \leq i \leq[(t-2 j+1) / 2]$. In the triple one, putting $k=i^{\prime}, i+j-k=j^{\prime}, j-k=k^{\prime}$, we see that $i=j^{\prime}-k^{\prime}, j=i^{\prime}+k^{\prime}$ and that $1 \leq i \leq[(t-1) / 2], 1 \leq j \leq[(t-2 i+1) / 2], 0 \leq k \leq j-1$ is equivalent to $0 \leq i^{\prime} \leq[(t-3) / 2], 2 \leq j^{\prime} \leq\left[\left(t-2 i^{\prime}+1\right) / 2\right], 1 \leq k^{\prime} \leq j^{\prime}-1$. Hence,

$$
\begin{aligned}
& \sum_{i=1}^{[t / 2]} E_{(i+1) \delta-\alpha_{1}} T_{\varpi_{1}}\left(\left[D_{(s-i) \alpha_{0}+(t-2 i) \alpha_{1}}^{-}, E_{\alpha_{1}}\right]_{q^{-2 s+t-1}}\right)[2 i+1]_{1} q^{(-2 s+t) i} \theta(t \geq 2) \\
& =\sum_{i=1}^{[(t+1) / 2]} E_{(i+1) \delta-\alpha_{1}} T_{\varpi_{1}}\left(D_{(s-i) \alpha_{0}+(t-2 i+1) \alpha_{1}}^{-}\right)[t-2 i+1]_{1}[2 i+1]_{1} q^{(-2 s+t) i} \\
& \quad+\sum_{i=1}^{[(t-1) / 2]} \sum_{j=1}^{[(t-2 j+1) / 2]} \tilde{\psi}_{i} E_{(j+1) \delta-\alpha_{1}} T_{\varpi_{1}}\left(D_{(s-i-j) \alpha_{0}+(t-2 i-2 j+1) \alpha_{1}}^{-}\right) \\
& \quad \times[2 j+1]_{1} q_{1}^{(-2 s+t)(2 i+2 j+1)+2(s-j)+1} \theta(t \geq 3) \\
& \quad+\sum_{i=0}^{[(t-3) / 2][(t-2 i+1) / 2]} \sum_{j=2}^{j-1} \sum_{k=1}^{j} \tilde{\psi}_{i} E_{(j+1) \delta-\alpha_{1}} T_{\varpi_{1}}\left(D_{(s-i-j) \alpha_{0}+(t-2 i-2 j+1) \alpha_{1}}^{-}\right) \\
& \quad \times b_{2 k}[2 j-2 k+1]_{1} q_{1}^{(-2 s+t)(2 i+2 j+1)+2(s-j+k)+1}\left(1+q^{-1}\right) \theta(t \geq 3) .
\end{aligned}
$$

Using Lemma 3.17 (1), we see that the last term is equal to

$$
\begin{aligned}
& \sum_{i=0}^{[(t-3) / 2]} \sum_{j=2}^{[(t-2 i+1) / 2]} \tilde{\psi}_{i} E_{(j+1) \delta-\alpha_{1}} T_{\varpi_{1}}\left(D_{(s-i-j) \alpha_{0}+(t-2 i-2 j+1) \alpha_{1}}^{-}\right) \\
& \quad \times\left(\left(q^{j}-q^{-j}\right)[2 j+1]_{1}-b_{2 j}\left(1+q^{-1}\right)\right) q_{1}^{(-2 s+t)(2 i+2 j+1)+2 s+1} \theta(t \geq 3) .
\end{aligned}
$$

Here, we can include the case where $j=1$, in which the factor $\left(\left(q^{j}-q^{-j}\right)[2 j\right.$ $\left.+1]_{1}-b_{2 j}\left(1+q^{-1}\right)\right)$ vanishes, and the case where $i=[(t-1) / 2](=[(t-3) / 2]$ $+1)$, in which we have $j=1$ since $[(t+1) / 2]-[(t-1) / 2]=1$, so that we can also include the case where $t=1,2$. Hence, applying $(2)_{s}$ to the definition of $B_{1}$, we have

$$
B_{1}=T_{\varpi_{1}}\left(D_{s \alpha_{0}+t \alpha_{1}}^{-}\right)[4 s-t+1]_{1}[t]_{1}\left(q_{1}-q_{1}^{-1}\right)
$$




$$
\begin{aligned}
& +\left(q_{1}-q_{1}^{-1}\right) \sum_{i=1}^{[t / 2]} \tilde{\psi}_{i} T_{\varpi_{1}}\left(D_{(s-i) \alpha_{0}+(t-2 i) \alpha_{1}}^{-}\right) \\
& \times\left([4 s-t+1]_{1} q_{1}^{(-2 s+t-1)(2 i+1)+2 s+1}-[2 i+1]_{1} q^{(-2 s+t)(i+1)}\right) \theta(t \geq 2) \\
& -\sum_{j=1}^{[(t+1) / 2]} E_{(j+1) \delta-\alpha_{1}} T_{\varpi_{1}}\left(D_{(s-j) \alpha_{0}+(t-2 j+1) \alpha_{1}}^{-}\right) \\
& \times[t-2 j+1]_{1}[2 j+1]_{1} q^{(-2 s+t) j}\left(q_{1}-q_{1}^{-1}\right) \\
& -\sum_{i=1} \sum_{j=1}^{[(t-1) / 2][(t-2 i+1) / 2]} \tilde{\psi}_{i} E_{(j+1) \delta-\alpha_{1}} T_{\varpi_{1}}\left(D_{(s-i-j) \alpha_{0}+(t-2 i-2 j+1) \alpha_{1}}^{-}\right) \\
& \times[2 j+1]_{1} q_{1}^{(-2 s+t)(2 i+2 j+1)+2(s-j)+1}\left(q_{1}-q_{1}^{-1}\right) \theta(t \geq 3) \\
& -\sum_{i=0}^{[(t-1) / 2][(t-2 i+1) / 2]} \sum_{j=1} \tilde{\psi}_{i} E_{(j+1) \delta-\alpha_{1}} T_{\varpi_{1}}\left(D_{(s-i-j) \alpha_{0}+(t-2 i-2 j+1) \alpha_{1}}^{-}\right) \\
& \times\left(\left(q^{j}-q^{-j}\right)[2 j+1]_{1}-b_{2 j}\left(1+q^{-1}\right)\right) q_{1}^{(-2 s+t)(2 i+2 j+1)+2 s+1}\left(q_{1}-q_{1}^{-1}\right) .
\end{aligned}
$$

It is now easy to see that $A_{1}=B_{1}$. We obtain (5) for $s$.

The proposition is proved.

Definition 7.6. For $n, r \geq 0$, we define the elements $D_{n \delta+r \alpha_{1}}^{+}$of $\mathbf{U}_{\mathbf{Z}}^{+}(>) \cap \mathbf{U}_{n \delta+r \alpha_{1}}^{+}$by $D_{n \delta}^{+}=\delta_{n, 0}, D_{r \alpha_{1}}^{+}=E_{\alpha_{1}}^{(r)}$, and $D_{n \delta+r \alpha_{1}}^{+}=\sum_{i=1}^{\min (r, 2 n)} E_{\alpha_{1}}^{(r-i)}\left(T_{1}^{-1} *\right)\left(D_{n \alpha_{0}+(2 n-i) \alpha_{1}}^{-}\right) q_{1}^{-(2 n-i)(r-i)} \quad$ for $n, r \geq 1$

Example 7.7. Let $n \geq 0$. Then, $D_{n \delta+\alpha_{1}}^{+}=E_{n \delta+\alpha_{1}}$.

The following is a consequence of Proposition 7.5 (5) and will be used in the next section.

Corollary 7.8. Let $n, r \geq 0$. Then,

$$
\sum_{i=0}^{n} D_{(n-i) \delta+r \alpha_{1}}^{+} E_{i \delta+\alpha_{1}}[2 i+1]_{1} q^{-i r}=D_{n \delta+(r+1) \alpha_{1}}^{+}[2 n+r+1]_{1} .
$$

Proof. We can assume that $n \geq 1$. We rewrite the left hand side, which 
is denoted by LHS. By Definition 7.6, we have

$$
\begin{aligned}
\text { LHS }= & E_{\alpha_{1}}^{(r)} E_{n \delta+\alpha_{1}}[2 n+1]_{1} q^{-n r} \\
& +\sum_{i=0}^{n-1} \sum_{j=1}^{\min (r, 2 n-2 i)} E_{\alpha_{1}}^{(r-j)}\left(T_{1}^{-1} *\right)\left(D_{(n-i) \alpha_{0}+(2 n-2 i-j) \alpha_{1}}^{-}\right) E_{i \delta+\alpha_{1}} \\
& \times[2 i+1]_{1} q_{1}^{(-2 n+2 i+j)(r-j)} q^{-i r} .
\end{aligned}
$$

We rewrite the $l=0$ part. Using Proposition 7.5 (5), we have

$$
\begin{aligned}
&\left(T_{1}^{-1} *\right)\left(D_{n \alpha_{0}+(2 n-j) \alpha_{1}}^{-}\right) E_{\alpha_{1}} \\
&=\left(T_{1}^{-1} *\right)\left(\left(T_{1}^{-1} *\right)\left(E_{\alpha_{1}}\right) D_{n \alpha_{0}+(2 n-j) \alpha_{1}}^{-}\right) \\
&=\left(T_{1}^{-1} * T_{\varpi_{1}}^{-1} T_{\varpi_{1}}\right)\left(\left(T_{1}^{-1} *\right)\left(E_{\alpha_{1}}\right) D_{n \alpha_{0}+(2 n-j) \alpha_{1}}^{-}\right) \\
&=\left(T_{1}^{-1} * T_{\varpi_{1}}^{-1}\right)\left(E_{\delta-\alpha_{1}} T_{\varpi_{1}}\left(D_{n \alpha_{0}+(2 n-j) \alpha_{1}}^{-}\right)\right) \\
&=\left(T_{1}^{-1} * T_{\varpi_{1}}^{-1}\right)\left(T_{\varpi_{1}}\left(D_{n \alpha_{0}+(2 n-j) \alpha_{1}}^{-}\right) E_{\delta-\alpha_{1}} q^{j}\right. \\
&+T_{\varpi_{1}}\left(D_{n \alpha_{0}+(2 n-j-1) \alpha_{1}}^{-}\right)[2 n+j+1]_{1} \theta(j \leq 2 n-1) \\
& \quad[(2 n-j) / 2] \\
& \quad \sum_{k=1}^{[} E_{(k+1) \delta-\alpha_{1}} T_{\varpi_{1}}\left(D_{(n-k) \alpha_{0}+(2 n-j-2 k) \alpha_{1}}^{-}\right) \\
&\left.\quad \times[2 k+1]_{1} q^{-j k} \theta(j \leq 2 n-2)\right) \\
&=E_{\alpha_{1}}\left(T_{1}^{-1} *\right)\left(D_{n \alpha_{0}+(2 n-j) \alpha_{1}}^{-}\right) q^{j} \\
&+\left(T_{1}^{-1} *\right)\left(D_{n \alpha_{0}+(2 n-j-1) \alpha_{1}}^{-}\right)[2 n+j+1]_{1} \theta(j \leq 2 n-1) \\
& {[(2 n-j) / 2] } \\
&- \sum_{k=1}\left(T_{1}^{-1} *\right)\left(E_{k \delta-\alpha_{1}} D_{(n-k) \alpha_{0}+(2 n-j-2 k) \alpha_{1}}^{-}\right) \\
& \times[2 k+1]_{1} q^{-j k} \theta(j \leq 2 n-2) .
\end{aligned}
$$

Hence,

$$
\begin{aligned}
\text { LHS }= & E_{\alpha_{1}}^{(r)} E_{n \delta+\alpha_{1}}[2 n+1]_{1} q^{-n r} \\
& +\sum_{i=1}^{n-1} \sum_{j=1}^{\min (r, 2 n-2 i)} E_{\alpha_{1}}^{(r-j)}\left(T_{1}^{-1} *\right)\left(E_{i \delta-\alpha_{1}} D_{(n-i) \alpha_{0}+(2 n-2 i-j) \alpha_{1}}^{-}\right) \\
& \times[2 i+1]_{1} q_{1}^{(-2 n+2 i+j)(r-j)} q^{-i r}
\end{aligned}
$$




$$
\begin{aligned}
& +\sum_{j=1}^{\min (r, 2 n)} E_{\alpha_{1}}^{(r-j)} E_{\alpha_{1}}\left(T_{1}^{-1} *\right)\left(D_{n \alpha_{0}+(2 n-j) \alpha_{1}}^{-}\right) q^{j} q_{1}^{(-2 n+j)(r-j)} \\
& +\sum_{j=1}^{\min (r, 2 n-1)} E_{\alpha_{1}}^{(r-j)}\left(T_{1}^{-1} *\right)\left(D_{n \alpha_{0}+(2 n-j-1) \alpha_{1}}^{-}\right)[2 n+j+1]_{1} q_{1}^{(-2 n+j)(k-j)} \\
& -\sum_{j=1}^{\min (r, 2 n-2)} \sum_{k=1}^{[(2 n-j) / 2]} E_{\alpha_{1}}^{(r-j)}\left(T_{1}^{-1} *\right)\left(E_{k \delta-\alpha_{1}} D_{(n-k) \alpha_{0}+(2 n-j-2 k) \alpha_{1}}^{-}\right) \\
& \times[2 k+1]_{1} q^{-j k} q_{1}^{(-2 n+j)(r-j)} .
\end{aligned}
$$

By Lemma $7.2(4)$, the first term is equal to $E_{\alpha_{1}}^{(r)}\left(T_{1}^{-1} *\right)\left(D_{n \alpha_{0}+(2 n-1) \alpha_{1}}^{-}\right)[2 n+$ $1]_{1} q_{1}^{-2 n k}$, which can be added to the fourth one for $i=0$. In the second one, we see that $1 \leq i \leq n-1,1 \leq j \leq \min (r, 2 n-2 i)$ is equivalent to $1 \leq j \leq \min (r, 2 n-2), 1 \leq i \leq[(2 n-j) / 2]$, so that it cancels out with the fifth one. Hence, replacing $j$ by $j+1$ in the third one, we have

$$
\begin{aligned}
\text { LHS }= & \sum_{j=0}^{\min (r, 2 n-1)} E_{\alpha_{1}}^{(r-j)}\left(T_{1}^{-1} *\right)\left(D_{n \alpha_{0}+(2 n-j-1) \alpha_{1}}^{-}\right) \\
& \times\left([r-j]_{1} q^{j+1} q_{1}^{(-2 n+j+1)(r-j-1)}+[2 n+j+1]_{1} q_{1}^{(-2 n+j)(r-j)}\right) \\
= & \sum_{i=0}^{\min (r, 2 n-1)} E_{\alpha_{1}}^{(r-i)}\left(T_{1}^{-1} *\right)\left(D_{n \alpha_{0}+(2 n-j-1) \alpha_{1}}^{-}\right)[2 n+r+1]_{1} q_{1}^{(-2 n+j+1)(r-j)} \\
= & \sum_{j=1}^{\min (r+1,2 n)} E_{\alpha_{1}}^{((r+1)-j)}\left(T_{1}^{-1} *\right)\left(D_{n \alpha_{0}+(2 n-j) \alpha_{1}}^{-}\right) \\
& \times q_{1}^{(-2 n+j)((r+1)-j)}[2 n+r+1]_{1} \\
= & D_{n \delta+(r+1) \alpha_{1}}^{+}[2 n+r+1]_{1} .
\end{aligned}
$$

The corollary is proved.

\section{$\S 8$. Construction of Basis II}

Definition 8.1. For $n, r \geq 0$, we define the elements $D_{n \delta+r \alpha_{1}}^{\geq 0}$ of $\mathbf{U}_{\mathbf{Z}}^{+}(>) \mathbf{U}_{\mathbf{Z}}^{+}(0) \cap \mathbf{U}_{n \delta+r \alpha_{1}}^{+}$by

$$
D_{n \delta+r \alpha_{1}}^{\geq 0}=\sum_{i=0}^{n} D_{(n-i) \delta+r \alpha_{1}}^{+} P_{i} q^{-i r} .
$$

Example 8.2. Let $n, k \geq 0$. Then, $D_{n \delta}^{\geq 0}=P_{n}, D_{k \alpha_{1}}^{\geq 0}=E_{\alpha_{1}}^{(k)}$. 
The following is a consequence of Corollary 7.8 .

Lemma 8.3. Let $n, r \geq 0$. Then,

$$
D_{n \delta+r \alpha_{1}}^{\geq 0} E_{\alpha_{1}}=\sum_{i=0}^{n} D_{(n-i) \delta+(r+1) \alpha_{1}}^{+} P_{i}[2 n-2 i+r+1]_{1} q^{-i r} .
$$

Proof. By Definition 8.1 and Lemma 4.15, the left hand side is equal to

$$
\begin{aligned}
& \sum_{i=0}^{n} D_{(n-i) \delta+r \alpha_{1}}^{+} P_{i} E_{\alpha_{1}} q^{-i r} \\
& \quad=\sum_{i=0}^{n} \sum_{j=0}^{i} D_{(n-i) \delta+r \alpha_{1}}^{+} E_{(i-j) \delta+\alpha_{1}} P_{j}[2 i-2 j+1]_{1} q^{-i r} .
\end{aligned}
$$

Putting $j=i^{\prime}, i-j=j^{\prime}$, we see that $i=i^{\prime}+j^{\prime}$ and that $0 \leq i \leq n, 0 \leq j \leq i$ is equivalent to $0 \leq i^{\prime} \leq n, 0 \leq j^{\prime} \leq n-i^{\prime}$. Thus, this is equal to

$$
\sum_{i=0}^{n} \sum_{j=0}^{n-i} D_{(n-i-j) \delta+r \alpha_{1}}^{+} E_{j \delta+\alpha_{1}} P_{i}[2 j+1]_{1} q^{-(i+j) r} .
$$

Applying Corollary 7.8, we obtain the lemma.

Lemma 8.4. Let $n \geq 1, r \geq 0$. Then,

$$
\sum_{i=1}^{n} D_{(n-i) \delta+r \alpha_{1}}^{\geq 0} \tilde{\psi}_{i} q^{i(-r+1)}=\sum_{i=1}^{n} D_{(n-i) \delta+r \alpha_{1}}^{+} P_{i}[2 i]_{1} q^{i(-r+1)} .
$$

Proof. By Definition 8.1, the left hand side is equal to

$$
\sum_{i=1}^{n} \sum_{j=0}^{n-i} D_{(n-i-j) \delta+r \alpha_{1}}^{+} P_{j} \tilde{\psi}_{i} q^{-j r} q^{i(-r+1)} .
$$

Putting $i=i^{\prime}-j$, we see that $1 \leq i \leq n, 0 \leq j \leq i-1$ is equivalent to $1 \leq i^{\prime} \leq n, 0 \leq j \leq i^{\prime}-1$. Thus, this is equal to

$$
\sum_{i=1}^{n} \sum_{j=0}^{i-1} D_{(n-i) \delta+r \alpha_{1}}^{+} P_{j} \tilde{\psi}_{i-j} q^{-j} q^{i(-r+1)} .
$$

Applying Definition 4.9, we obtain the lemma.

Using Proposition 7.5 (2) and Lemma 8.3, we prove the following. 
Theorem 8.5. Let $r, s \geq 0$. Then,

$$
\begin{aligned}
E_{\alpha_{0}}^{(s)} & E_{\alpha_{1}}^{(r)} \\
& =\sum_{t=0}^{\min (2 s-1, r)} \sum_{i=0}^{[t / 2]} D_{i \delta+(r-t) \alpha_{1}}^{\geq 0} D_{(s-i) \alpha_{0}+(t-2 i) \alpha_{1}}^{-} q^{i(r-2 s)} q_{1}^{(r-t)(-4 s+t)} \theta(s \geq 1) \\
& +D_{s \delta+(r-2 s) \alpha_{1}}^{\geq 0} \theta(r \geq 2 s) .
\end{aligned}
$$

Note that if $s \geq 1$, then $0 \leq t \leq \min (2 s-1, r), 0 \leq i \leq[t / 2]$ is equivalent to $0 \leq i \leq \min (s-1,[r / 2]), 2 i \leq t \leq \min (2 s-1, r)$.

Proof. We argue by the induction on $r$. The case where $r=0$ is clear. Assuming the case for $r$, we shall prove the case for $r+1$. We can assume that $s \geq 1$. Using the induction hypothesis and Proposition 7.5 (2), we have

$$
\begin{aligned}
E_{\alpha_{0}}^{(s)} E_{\alpha_{1}}^{(r)} E_{\alpha_{1}}= & \sum_{t=0}^{\min (2 s-1, r)} \sum_{i=0}^{[t / 2]} D_{i \delta+(r-t) \alpha_{1}}^{\geq 0}\left(E_{\alpha_{1}} D_{(s-i) \alpha_{0}+(t-2 i) \alpha_{1}}^{-} q^{-2 s+t}\right. \\
& +D_{(s-i) \alpha_{0}+(t-2 i+1) \alpha_{1}}^{-}[t-2 i+1]_{1} \\
& +\sum_{j=1}^{[(t-2 i+1) / 2]} \tilde{\psi}_{j} D_{(s-i-j) \alpha_{0}+(t-2 i-2 j+1) \alpha_{1}}^{-} \\
& \left.\times q_{1}^{(-2 s+t)(2 j+1)+2(s-i)+1}\right) \\
& \times q^{i(r-2 s)} q_{1}^{(r-t)(-4 s+t)}+D_{s \alpha_{0}+(r-2 s) \alpha_{1}}^{\geq 0} E_{\alpha_{1}} \theta(r \geq 2 s) .
\end{aligned}
$$

Hence, by Lemma 8.3, if we set

$$
\begin{aligned}
A= & \sum_{t=0}^{\min (2 s-1, r)} \sum_{i=0}^{[t / 2]} \sum_{j=0}^{i} D_{(i-j) \delta+(r-t+1) \alpha_{1}}^{+} P_{j} D_{(s-i) \alpha_{0}+(t-2 i) \alpha_{1}}^{-} \\
& \times[2 i-2 j+r-t+1]_{1} q^{-j(r-t)} q^{-2 s+t} q^{i(r-2 s)} q_{1}^{(r-t)(-4 s+t)}, \\
B= & \sum_{t=0}^{\min (2 s-1, r)} \sum_{i=0}^{[t / 2]} D_{i \delta+(r-t) \alpha_{1}}^{\geq 0} D_{(s-i) \alpha_{0}+(t-2 i+1) \alpha_{1}}^{-} \\
& \times[t-2 i+1]_{1} q^{i(r-2 s)} q_{1}^{(r-t)(-4 s+t)}, \\
C= & \sum_{t=0}^{\min (2 s-1, r)[t / 2]} \sum_{i=0}^{[(t-2 i+1) / 2]} \sum_{j=1}^{\geq} D_{i \delta+(r-t) \alpha_{1}}^{\geq 0} \tilde{\psi}_{j} D_{(s-i-j) \alpha_{0}+(t-2 i-2 j+1) \alpha_{1}}^{-} \\
& \times q_{1}^{(-2 s+t)(2 j+1)+2(s-i)+1} q^{i(r-2 s)} q_{1}^{(r-t)(-4 s+t)},
\end{aligned}
$$




$$
D=\sum_{j=0}^{s} D_{(s-j) \delta+(r-2 s+1) \alpha_{1}}^{+} P_{j}[r-2 j+1]_{1} q^{-j(r-2 s)} \theta(r \geq 2 s),
$$

then we have

$$
E_{\alpha_{0}}^{(s)} E_{\alpha_{1}}^{(r+1)}[r+1]_{1}=A+B+C+D
$$

Note that in the above expression of $A$, on account of the factor $[2 i-2 j+r-$ $t+1]_{1}$, the range of $t$ can be changed to $0 \leq t \leq \min (2 s-1, r+1)$. Also, in the above expression of $D$, on account of the factor $[r-2 j+1]_{1}$, the factor $\theta(r \geq 2 s)$ can be changed to $\theta(r+1 \geq 2 s)$. As for $B$, by Definition 8.1, we have

$$
\begin{aligned}
B= & \sum_{t=0}^{\min (2 s-1, r)} \sum_{i=0}^{[t / 2]} \sum_{j=0}^{i} D_{(i-j) \delta+(r-t) \alpha_{1}}^{+} P_{j} D_{(s-i) \alpha_{0}+(t-2 i+1) \alpha_{1}}^{-} \\
& \times[t-2 i+1]_{1} q^{-j(r-t)} q^{i(r-2 s)} q_{1}^{(r-t)(-4 s+t)} .
\end{aligned}
$$

Here, on account of the factor $[t-2 i+1]_{1}$, we can change the range of $i$ to $0 \leq i \leq[(t+1) / 2]$. We rewrite $C$. Putting $i+j=i^{\prime}$, we see that $i=i^{\prime}-j$ and that $0 \leq i \leq[t / 2], 1 \leq j \leq[(t-2 i+1) / 2]$ is equivalent to $0 \leq i^{\prime} \leq$ $[(t+1) / 2], 1 \leq j \leq i^{\prime}$. Thus,

$$
\begin{aligned}
C= & \sum_{t=0}^{\min (2 s-1, r)} \sum_{i=0}^{[(t+1) / 2]} \sum_{j=1}^{i} D_{(i-j) \delta+(r-t) \alpha_{1}}^{\geq 0} \tilde{\psi}_{j} D_{(s-i) \alpha_{0}+(t-2 i+1) \alpha_{1}}^{-} \\
& \times q_{1}^{(-2 s+t)(2 j+1)+2(s-i+j)+1} q^{(i-j)(r-2 s)} q_{1}^{(r-t)(-4 s+t)} .
\end{aligned}
$$

Applying Lemma 8.4, we have

$$
\begin{aligned}
C= & \sum_{t=0}^{\min (2 s-1, r)} \sum_{i=0}^{[(t+1) / 2]} \sum_{j=1}^{i} D_{(i-j) \delta+(r-t) \alpha_{1}}^{+} P_{j} D_{(s-i) \alpha_{0}+(t-2 i+1) \alpha_{1}}^{-} \\
& \times[2 j]_{1} q^{j(-r+t+1)} q_{1}^{t-2 i+1} q^{i(r-2 s)} q_{1}^{(r-t)(-4 s+t)} .
\end{aligned}
$$

Thus,

$$
\begin{aligned}
B+C= & \sum_{t=0}^{\min (2 s-1, r)} \sum_{i=0}^{[(t+1) / 2]} \sum_{j=0}^{i} D_{(i-j) \delta+(r-t) \alpha_{1}}^{+} P_{j} D_{(s-i) \alpha_{0}+(t-2 i+1) \alpha_{1}}^{-} \\
& \times[t-2 i+2 j+1]_{1} q^{j(-r+t+1)} q^{i(r-2 s)} q_{1}^{(r-t)(-4 s+t)} .
\end{aligned}
$$

Replacing $t$ by $t-1$, we have

$$
\begin{aligned}
B+C= & \sum_{t=1}^{\min (2 s, r+1)} \sum_{i=0}^{[t / 2]} \sum_{j=0}^{i} D_{(i-j) \delta+(r-t+1) \alpha_{1}}^{+} P_{j} D_{(s-i) \alpha_{0}+(t-2 i) \alpha_{1}}^{-} \\
& \times[t-2 i+2 j]_{1} q^{j(-r+t)} q^{i(r-2 s)} q_{1}^{(r-t+1)(-4 s+t-1)} .
\end{aligned}
$$


Here, on account of the factor $[t-2 i+2 j]_{1}$, we can include the case where $t=0$. Hence,

$$
\begin{aligned}
A+ & B+C \\
= & \sum_{t=0}^{\min (2 s-1, r+1)} \sum_{i=0}^{[t / 2]} \sum_{j=0}^{i} D_{(i-j) \delta+(r-t+1) \alpha_{1}}^{+} P_{j} D_{(s-i) \alpha_{0}+(t-2 i) \alpha_{1}}^{-} \\
& \times[r+1]_{1} q^{j(-r+t-1)} q^{i(r-2 s+1)} q_{1}^{(r-t+1)(-4 s+t)} \\
& +\sum_{j=0}^{s} D_{(s-j) \delta+(r-2 s+1) \alpha_{1}}^{+} P_{j}[2 j]_{1} q^{(s-j)(r-2 s)} q_{1}^{-(r-2 s+1)(2 s+1)} \theta(r+1 \geq 2 s) .
\end{aligned}
$$

By Definition 8.1, we see that the former term is equal to

$$
\sum_{t=0}^{\min (2 s-1, r+1)} \sum_{i=0}^{[t / 2]} D_{i \delta+(r-t+1) \alpha_{1}}^{\geq 0} D_{(s-i) \alpha_{0}+(t-2 i) \alpha_{1}}^{-}[r+1]_{1} q^{i(r-2 s+1)} q_{1}^{(r-t+1)(-4 s+t)}
$$

and that the latter plus $D$ is equal to

$$
\begin{gathered}
\sum_{j=0}^{s} D_{(s-j) \delta+(r-2 s+1) \alpha_{1}}^{+} P_{j}[r+1]_{1} q^{-j(r-2 s+1)} \theta(r+1 \geq 2 s) \\
=D_{s \alpha_{0}+(r-2 s+1) \alpha_{1}}^{\geq 0}[r+1]_{1} \theta(r+1 \geq 2 s)
\end{gathered}
$$

Thus, we obtain the case for $r+1$. The theorem is proved.

Corollary 8.6. We have $\mathbf{U}_{\mathbf{Z}}^{+}(0) \subset \mathbf{U}_{\mathbf{Z}}^{+}$.

Proof. It is enough to show that $P_{s} \in \mathbf{U}_{\mathbf{Z}}^{+}$for $s \geq 0$, but it follows from Theorem 8.5 with $r=2 s$ by the induction on $s$.

We shall prove that $V_{\mathbf{Z}}$ is closed under multiplication (Proposition 8.13). First, we shall prove that both of $\mathbf{U}_{\mathbf{Z}}^{+}(>) \mathbf{U}_{\mathbf{Z}}^{+}(0)$ and $\mathbf{U}_{\mathbf{Z}}^{+}(0) \mathbf{U}_{\mathbf{Z}}^{+}(<)$are closed under multiplication (Corollary 8.9).

Proposition 8.7. Let $s \geq 0$. Then,

(1) $D_{s \alpha_{0}+r \alpha_{1}}^{-} E_{\alpha_{1}}^{(k)} \in V_{\mathbf{Z}}$ for $r, k \geq 0$,

(2) $P_{s} E_{n \delta+\alpha_{1}}^{(k)} \in \mathbf{U}_{\mathbf{Z}}^{+}\left(>; n \delta+\alpha_{1}\right) \mathbf{U}_{\mathbf{Z}}^{+}(0 ; s)$ for $n, k \geq 0$,

(3) $E_{\alpha_{0}}^{(k)} D_{s \delta+r \alpha_{1}}^{\geq 0} \in V_{\mathbf{Z}}$ for $k, r \geq 0$,

(4) $P_{s} E_{(2 n+2) \delta-\alpha_{0}}^{(k)} \in \mathbf{U}_{\mathbf{Z}}^{+}\left(>;(2 n+2) \delta-\alpha_{0}\right) \mathbf{U}_{\mathbf{Z}}^{+}(0 ; s)$ for $n, k \geq 0$. 
Proof. We denote by $(\mathrm{a})_{r}$ the statement (a) for $s=0,1, \ldots, r$. First, we prove the following.

Sublemma 8.8. Let $s \geq 1$ and assume that $(2)_{s-1}$ and $(4)_{s-1}$ are valid. Let $\alpha \in R_{r e}^{+}(>), x \in \mathbf{U}_{\mathbf{Z}}^{+, h}(>; \alpha)$ and let $y \in \mathbf{U}_{\mathbf{Z}}^{+, h}(0 ; s-1)$. Then, $y x=\sum_{i} x_{i} y_{i}$ for some $x_{i} \in \mathbf{U}_{\mathbf{Z}}^{+, h}(>; \alpha), y_{i} \in \mathbf{U}_{\mathbf{Z}}^{+, h}(0 ; s-1)$ with $\mathrm{h}\left(x_{i}\right)=\mathrm{h}(x), \mathrm{i}\left(y_{i}\right) \leq \mathrm{i}(y)$.

Proof. By the same argument in the proof of Proposition 4.17, the sublemma is reduced to the case where $x=E_{n \delta+\alpha_{1}}^{(k)}$ with $n \delta+\alpha_{1} \geq \alpha$ or $x=$ $E_{(2 n+2) \delta-\alpha_{0}}^{(k)}$ with $(2 n+2) \delta-\alpha_{0} \geq \alpha$, and $y=P_{i}$ for $1 \leq i \leq s-1$, but it follows from $(2)_{s-1}$ and $(4)_{s-1}$.

We shall prove $(1)_{s}-(4)_{s}$ at once by the induction on $s$. If $s=0$, then (3) follows from Theorem 8.5, and the others are clear. Now, assume that $s \geq 1$.

$(1)_{s}$ We argue by the induction on $r$. The case where $r=0$ follows from Theorem 8.5. We can assume that $1 \leq r \leq 2 s-1$. By Theorem 8.5, we have

$$
\begin{aligned}
& E_{\alpha_{0}}^{(s)} E_{\alpha_{1}}^{(r)} E_{\alpha_{1}}^{(k)} \\
& =\sum_{i=1}^{[r / 2]} \sum_{t=2 i}^{r} D_{i \delta+(r-t) \alpha_{1}}^{\geq 0} D_{(s-i) \alpha_{0}+(t-2 i) \alpha_{1}}^{-} E_{\alpha_{1}}^{(k)} q^{i(r-2 s)} q_{1}^{(r-t)(-4 s+t)} \theta(r \geq 2) \\
& \quad+\sum_{t=0}^{r-1} E_{\alpha_{1}}^{(r-t)} D_{s \alpha_{0}+t \alpha_{1}}^{-} E_{\alpha_{1}}^{(k)} q_{1}^{(r-t)(-4 s+t)}+D_{s \alpha_{0}+r \alpha_{1}}^{-} E_{\alpha_{1}}^{(k)} .
\end{aligned}
$$

The left hand side is equal to $E_{\alpha_{0}}^{(s)} E_{\alpha_{1}}^{(r+k)}\left[\begin{array}{c}r+k \\ k\end{array}\right]_{1}$, which belongs to $V_{\mathbf{Z}}$ by Theorem 8.5. The first term on the right hand side belongs to $V_{\mathbf{Z}}$, since for $1 \leq i \leq[r / 2], 2 i \leq t \leq r$, we have $D_{(s-i) \alpha_{0}+(t-2 i) \alpha_{1}}^{-} E_{\alpha_{1}}^{(k)} \in V_{\mathbf{Z}}$ by $(1)_{s-1}$; hence, $D_{i \delta+(r-t) \alpha_{1}}^{\geq 0} D_{(s-i) \alpha_{0}+(t-2 i) \alpha_{1}}^{-} E_{\alpha_{1}}^{(k)} \in V_{\mathbf{Z}}$ by Sublemma 8.8 and Proposition $4.17(2)$. The second one on the right hand side belongs to $V_{\mathbf{Z}}$ by the induction hypothesis. Thus, $D_{s \alpha_{0}+r \alpha_{1}}^{-} E_{\alpha_{1}}^{(k)} \in V_{\mathbf{Z}}$. We obtain (1) for $s$.

$(2)_{s}$ By Theorem 8.5, we have

$$
\begin{aligned}
& E_{\alpha_{0}}^{(s)} E_{\alpha_{1}}^{(2 s)} E_{\alpha_{1}}^{(k)} \\
& \quad=\sum_{t=0}^{2 s-1} \sum_{i=0}^{[t / 2]} D_{i \delta+(2 s-t) \alpha_{1}}^{\geq 0} D_{(s-i) \alpha_{0}+(t-2 i) \alpha_{1}}^{-} E_{\alpha_{1}}^{(k)} q_{1}^{(2 s-t)(-4 s+t)}+P_{s} E_{\alpha_{1}}^{(k)} .
\end{aligned}
$$

The left hand side is equal to $E_{\alpha_{0}}^{(s)} E_{\alpha_{1}}^{(2 s+k)}\left[\begin{array}{c}2 s+k \\ k\end{array}\right]_{1}$, which belongs to $V_{\mathbf{Z}}$ by Theorem 8.5. The first term on the right hand side belongs to $V_{\mathbf{Z}}$, since for $0 \leq t \leq 2 s-1,0 \leq i \leq[t / 2]$, we have $D_{(s-i) \alpha_{0}+(t-2 i) \alpha_{1}}^{-} E_{\alpha_{1}}^{(k)} \in V_{\mathbf{Z}}$ by virtue 
of $(1)_{s}$; hence, $D_{i \delta+(2 s-t) \alpha_{1}}^{\geq 0} D_{(s-i) \alpha_{0}+(t-2 i) \alpha_{1}}^{-} E_{\alpha_{1}}^{(k)} \in V_{\mathbf{Z}}$ by Sublemma 8.8 and Corollary 4.6. Hence, by Proposition 4.17, we have $P_{s} E_{n \delta+\alpha_{1}}^{(k)} \in V_{\mathbf{Z}} \cap \mathbf{U}^{+}(>$ $\left.; n \delta+\alpha_{1}\right) \mathbf{U}^{+}(0 ; s)=\mathbf{U}_{\mathbf{Z}}^{+}\left(>; n \delta+\alpha_{1}\right) \mathbf{U}_{\mathbf{Z}}^{+}(0 ; s)$. We obtain $(2)$ for $s$.

$(3)_{s}$ By Theorem 8.5, we have

$$
\begin{aligned}
& E_{\alpha_{0}}^{(k)} E_{\alpha_{0}}^{(s)} E_{\alpha_{1}}^{(r+2 s)} \\
& =\sum_{i=0}^{s-1} \sum_{t=2 i}^{2 s-1} E_{\alpha_{0}}^{(k)} D_{i \delta+(r+2 s-t) \alpha_{1}}^{\geq 0} D_{(s-i) \alpha_{0}+(t-2 i) \alpha_{1}}^{-} q^{i r} q_{1}^{(-4 s+t)(r+2 s-t)} \\
& \quad+E_{\alpha_{0}}^{(k)} D_{s \delta+r \alpha_{1}}^{\geq 0} .
\end{aligned}
$$

The left hand side is equal to $E_{\alpha_{0}}^{(k+s)} E_{\alpha_{1}}^{(r+2 s)}\left[\begin{array}{c}k+s \\ k\end{array}\right]_{0}$, which belongs to $V_{\mathbf{Z}}$ by Theorem 8.5. The first term on the right hand side belongs to $V_{\mathbf{Z}}$, since for $0 \leq i \leq s-1,2 i \leq t \leq 2 s-1$, we have $E_{\alpha_{0}}^{(k)} D_{i \delta+(r+2 s-t) \alpha_{1}}^{\geq 0} \in V_{\mathbf{Z}}$ by $(3)_{s-1}$; thus, $E_{\alpha_{0}}^{(k)} D_{i \delta+(r+2 s-t) \alpha_{1}}^{\geq 0} D_{(s-i) \alpha_{0}+(t-2 i) \alpha_{1}}^{-} \in V_{\mathbf{Z}}$ by Corollary 4.7 (4). Hence, $E_{\alpha_{0}}^{(k)} D_{s \delta+r \alpha_{1}}^{\geq 0} \in V_{\mathbf{Z}}$. We obtain (3) for $s$.

$(4)_{s}$ By Theorem 8.5, we have

$$
\begin{aligned}
& E_{\alpha_{0}}^{(k)} E_{\alpha_{0}}^{(s)} E_{\alpha_{1}}^{(2 s)} \\
& =\sum_{t=0}^{2 s-1} \sum_{i=0}^{[t / 2]} E_{\alpha_{0}}^{(k)} D_{i \delta+(2 s-t) \alpha_{1}}^{\geq 0} D_{(s-i) \alpha_{0}+(t-2 i) \alpha_{1}}^{-} q_{1}^{(-4 s+t)(2 s-t)}+E_{\alpha_{0}}^{(k)} P_{s} .
\end{aligned}
$$

The left hand side is equal to $E_{\alpha_{0}}^{(k+s)} E_{\alpha_{1}}^{(2 s)}\left[\begin{array}{c}k+s \\ k\end{array}\right]_{0}$, which belongs to $V_{\mathbf{Z}}$ by Theorem 8.5. The first term on the right hand side belongs to $V_{\mathbf{Z}}$, since for $0 \leq t \leq 2 s-1,0 \leq i \leq[t / 2]$, we have $E_{\alpha_{0}}^{(k)} D_{i \delta+(2 s-t) \alpha_{1}}^{\geq 0} \in V_{\mathbf{Z}}$ by $(3)_{s-1}$; hence, $E_{\alpha_{0}}^{(k)} D_{i \delta+(2 s-t) \alpha_{1}}^{\geq 0} D_{(s-i) \alpha_{0}+(t-2 i) \alpha_{1}}^{-} \in V_{\mathbf{Z}}$ by Corollary 4.6 (4). Hence, by Proposition 4.17, we have $E_{\alpha_{0}}^{(k)} P_{s} \in V_{\mathbf{Z}} \cap \mathbf{U}^{+}(0 ; s) \mathbf{U}^{+}(<)=\mathbf{U}_{\mathbf{Z}}^{+}(0 ; s) \mathbf{U}_{\mathbf{Z}}^{+}(<)$. Applying $T_{\varpi_{1}}^{n}$, we have $E_{2 n \delta+\alpha_{0}}^{(k)} P_{s} \in \mathbf{U}_{\mathbf{Z}}^{+}(0 ; s) \mathbf{U}_{\mathbf{Z}}^{+}\left(<; 2 n \delta+\alpha_{0}\right)$ for $n \geq 0$. Applying $T_{1}^{-1} *$, we have $P_{s} E_{(2 n+2) \delta-\alpha_{0}}^{(k)} \in \mathbf{U}_{\mathbf{Z}}^{+}\left(>;(2 n+2) \delta-\alpha_{0}\right) \mathbf{U}_{\mathbf{Z}}^{+}(0 ; s)$ for $n \geq 0$. We obtain (4) for $s$.

The proposition is proved.

Corollary 8.9. Let $\alpha \in R_{r e}^{+}(>), \beta \in R_{r e}^{+}(<)$and let $n \geq 0$.

(1) Let $x \in \mathbf{U}_{\mathbf{Z}}^{+, h}(>; \alpha), y \in \mathbf{U}_{\mathbf{Z}}^{+, h}(0 ; n)$. Then, $y x=\sum_{i} x_{i} y_{i}$ for some $x_{i} \in$ $\mathbf{U}_{\mathbf{Z}}^{+, h}(>; \alpha), y_{i} \in \mathbf{U}_{\mathbf{Z}}^{+, h}(0 ; n)$ with $\mathrm{h}\left(x_{i}\right)=\mathrm{h}(x), \mathrm{i}\left(y_{i}\right) \leq \mathrm{i}(y)$.

(2) $\mathbf{U}_{\mathbf{Z}}^{+}(>; \alpha) \mathbf{U}_{\mathbf{Z}}^{+}(0 ; n)$ is closed under multiplication.

(3) Let $y \in \mathbf{U}_{\mathbf{Z}}^{+, h}(0 ; n), z \in \mathbf{U}_{\mathbf{Z}}^{+, h}(<; \beta)$. Then, $z y=\sum_{i} y_{i} z_{i}$ for some $y_{i} \in$ $\mathbf{U}_{\mathbf{Z}}^{+, h}(0 ; n), z_{i} \in \mathbf{U}_{\mathbf{Z}}^{+, h}(<; \beta)$ with $\mathrm{i}\left(y_{i}\right) \leq \mathrm{i}(y), \mathrm{h}\left(z_{i}\right)=\mathrm{h}(z)$. 
(4) $\mathbf{U}_{\mathbf{Z}}^{+}(0 ; n) \mathbf{U}_{\mathbf{Z}}^{+}(<; \beta)$ is closed under multiplication.

Proof. (1) is nothing but Sublemma 8.8. (2) follows from (1) by the same argument in the proof of Proposition 4.17 (2). Applying $T_{1}^{-1} *$ to (1) and (2), we obtain (3) and (4) respectively.

Corollary 8.10. Let $s, t, k \geq 0$. Then, $E_{\alpha_{0}}^{(k)} D_{s \alpha_{0}+t \alpha_{1}}^{+} \in V_{\mathbf{Z}}$.

Proof. By Corollary 8.9 (4), we have $V_{\mathbf{Z}} P_{n} \subset V_{\mathbf{Z}}$ for $n \geq 0$. Thus, the corollary follows from Proposition 8.7 (3) and Definition 8.1 by the induction on $s$.

Lemma 8.11. Let $n, k \geq 0$. Then,

(1) $D_{(n+1) k \alpha_{0}+(2 n+1) k \alpha_{1}}^{-}=E_{(n+1) \delta-\alpha_{1}}^{(k)}+\sum_{i} z_{i 1} z_{i 2}$ for some $z_{i j} \in \mathbf{U}_{\mathbf{Z}}^{+, h}(<)$ with $\mathrm{h}\left(z_{i j}\right) \leq-1$,

(2) $D_{(2 n+1) k \alpha_{0}+4 n k \alpha_{1}}^{-}=E_{2 n \delta+\alpha_{0}}^{(k)}+\sum_{i} z_{i 1} z_{i 2}$ for some $z_{i j} \in \mathbf{U}_{\mathbf{Z}}^{+, h}(<)$ with $\mathrm{h}\left(z_{i j}\right) \leq-1$.

Proof. This follows from Definition 7.1 by the induction on $n$.

Lemma 8.12. Let $n, k \geq 0$. Then,

(1) $D_{n k \delta+k \alpha_{1}}^{+}=E_{n \delta+\alpha_{1}}^{(k)}+\sum_{i} x_{i 1} x_{i 2}$ for some $x_{i j} \in \mathbf{U}_{\mathbf{Z}}^{+, h}(>)$ with $\mathrm{h}\left(x_{i j}\right) \geq 1$,

(2) $D_{(2 n+1) k \delta+2 k \alpha_{1}}^{+}=E_{2 n \delta+\alpha_{0}}^{(k)}+\sum_{i} x_{i 1} x_{i 2}$ for some $x_{i j} \in \mathbf{U}_{\mathbf{Z}}^{+, h}(>)$ with $\mathrm{h}\left(x_{i j}\right)$ $\geq 1$.

Proof. In view of Definition 7.6, we obtain the lemma by applying $T_{1}^{-1} *$ to Lemma 8.11.

Proposition 8.13. Let $\alpha \in R_{r e}^{+}(>), \beta \in R_{r e}^{+}(<)$and let $x \in \mathbf{U}_{\mathbf{Z}}^{+, h}(>$ $; \alpha), z \in \mathbf{U}_{\mathbf{Z}}^{+, h}(<; \beta)$. Then,

(1) $z x=\sum_{i} x_{i} y_{i} z_{i}$ for some $x_{i} \in \mathbf{U}_{\mathbf{Z}}^{+, h}(>; \alpha), y_{i} \in \mathbf{U}_{\mathbf{Z}}^{+, h}(0), z_{i} \in \mathbf{U}_{\mathbf{Z}}^{+, h}(<; \beta)$ with $\mathrm{h}\left(x_{i}\right) \leq \mathrm{h}(x), \mathrm{h}\left(z_{i}\right) \geq \mathrm{h}(z)$,

(2) $\mathbf{U}_{\mathbf{Z}}^{+}(>; \alpha) \mathbf{U}_{\mathbf{Z}}^{+}(0) \mathbf{U}_{\mathbf{Z}}^{+}(<; \beta)$ is closed under multiplication.

Proof. We prove (1) by the induction on $\mathrm{h}(x)-\mathrm{h}(z)$. We can assume that $\mathrm{h}(x) \geq 1$ and $\mathrm{h}(z) \leq-1$. By the same argument in the proof of Proposition 4.19 
(1), we see that (1) is reduced to the case where $x=E_{n \delta+\alpha_{1}}^{(k)}$ with $n \delta+\alpha_{1} \geq \alpha$ or $x=E_{(2 n+2) \delta-\alpha_{0}}^{(k)}$ with $(2 n+2) \delta-\alpha_{0} \geq \alpha$, and $z=E_{m \delta-\alpha_{1}}^{(l)}$ with $m \delta-\alpha_{1} \leq \beta$ or $z=E_{2 m \delta+\alpha_{0}}^{(l)}$ with $2 m \delta+\alpha_{0} \leq \beta$. By virtue of Proposition 4.19 , we only have to show that $z x \in V_{\mathbf{Z}}$ for these cases. The case where $x=E_{n \delta+\alpha_{1}}^{(k)}$ is reduced to the case where $x=E_{\alpha_{1}}^{(k)}$ by using $T_{\varpi_{1}}^{n}$, but it follows from Proposition 8.7 (1), Lemma 8.11, and the induction hypothesis by the same argument in the proof of Proposition 4.19 (1). The case where $x=E_{(2 n+2) \delta-\alpha_{0}}^{(k)}$ is reduced to the case where $x=E_{2 \delta-\alpha_{0}}^{(k)}$ and $z=E_{m \delta-\alpha_{1}}^{(l)}$ or $E_{2 m \delta+\alpha_{0}}^{(l)}$ by using $T_{\varpi_{1}}^{n}$, which in turn is reduced to the case where $z=E_{\alpha_{0}}^{(k)}$ and $x=E_{n \delta+\alpha_{1}}^{(l)}$ or $E_{(2 n+2) \delta-\alpha_{0}}^{(l)}$ by using $T_{1}^{-1} *$, but it follows from Corollary 8.10, Lemma 8.12, and the induction hypothesis by the same argument in the proof of Proposition 4.19 (1). (1) is proved. By the same argument in the proof of Proposition 4.19 (2), we obtain (2).

Theorem 8.14. Both of the following are $\mathbf{Z}\left[q_{1}, q_{1}^{-1}\right]$-bases of $\mathbf{U}_{\mathbf{Z}}^{+}$:

(1) $\left\{\mathbf{E}_{\mathbf{c}_{+}} \mathbf{E}_{\mathbf{c}_{\mathbf{0}}} \mathbf{E}_{\mathbf{c}_{-}} \mid \mathbf{c}_{+} \in \oplus_{i \in R_{r e}^{+}(>)} \mathbf{Z}_{\geq 0}^{(i)}, \mathbf{c}_{\mathbf{0}} \in \oplus_{n \geq 1} \mathbf{Z}_{\geq 0}^{(n)}, \mathbf{c}_{-} \in \oplus_{i \in R_{r e}^{+}(<)} \mathbf{Z}_{\geq 0}^{(i)}\right\}$,

(2) $\left\{\mathbf{E}_{\mathbf{c}_{+}} \mathbf{S}_{\lambda} \mathbf{E}_{\mathbf{c}_{-}} \mid \mathbf{c}_{+} \in \oplus_{i \in R_{r e}^{+}(>)} \mathbf{Z}_{\geq 0}^{(i)}, \lambda\right.$ is a partition, $\left.\mathbf{c}_{-} \in \oplus_{i \in R_{r e}^{+}(<)} \mathbf{Z}_{\geq 0}^{(i)}\right\}$.

The basis in (1) has the convexity (see Remark 6.20) and the one in (2) is quasi-orthonormal (see Lemma 6.21).

Proof. As is discussed at the end of Section 6, this follows from Corollary 8.6 and Proposition 8.13.

We shall prove that the basis in Theorem $8.14(2)$ is an integral crystal basis of $\mathbf{U}^{+}$. First, let us recall some properties of the canonical basis. We set $L=\left\{x \in \mathbf{U}_{\mathbf{Z}}^{+} \mid(x, x) \in \mathbf{A}\right\}$, which is a $\mathbf{Z}\left[q_{1}^{-1}\right]$-submodule of $\mathbf{U}_{\mathbf{Z}}^{+}$. Then we have $q_{1}^{-1} L=\left\{x \in \mathbf{U}_{\mathbf{Z}}^{+} \mid(x, x) \in q_{1}^{-1} \mathbf{A}\right\}$. Let $\pi$ be the canonical projection from $L$ to $L / q_{1}^{-1} L$.

Proposition 8.15 (see $[\mathrm{L}])$. Let $\mathbf{B}$ be the canonical basis of $\mathbf{U}^{+}$.

(1) $\mathbf{B}$ is quasi-orthonormal with respect to the inner product on $\mathbf{U}^{+}$, that is, $\left(b, b^{\prime}\right) \equiv \delta_{b, b^{\prime}} \bmod q_{1}^{-1} \mathbf{A}$ for $b, b^{\prime} \in \mathbf{B}$.

(2) Each element of $\mathbf{B}$ is homogeneous; thus, $\mathbf{B}=\sqcup_{\alpha \in Q^{+}}\left(\mathbf{B} \cap \mathbf{U}_{\alpha}^{+}\right)$.

(3) Each element of $\mathbf{B}$ is invariant under -.

(4) For $i \in I, k \geq 0$, we have $E_{\alpha_{i}}^{(k)} \in \mathbf{B}$. 
(5) Let $i \in I$ and let $x \in \mathbf{B}+q_{1}^{-1} L$. If ${ }_{i} r(x)=0$, then $E_{\alpha_{i}}^{(k)} x \in \mathbf{B}+q_{1}^{-1} L$ for $k \geq 0$.

(6) $\mathbf{B}$ is a $\mathbf{Q}\left(q_{1}\right)$-basis of $\mathbf{U}^{+}$and a $\mathbf{Z}\left[q_{1}, q_{1}^{-1}\right]$-basis of $\mathbf{U}_{\mathbf{Z}}^{+}$.

(7) $\mathbf{B}$ is a $\mathbf{Z}\left[q_{1}^{-1}\right]$-basis of $L$ and a $\mathbf{Z}\left[q_{1}\right]$-basis of $\bar{L}$.

(8) $\mathbf{B}$ is a $\mathbf{Z}$-basis of $L \cap \bar{L}$ and the restriction of $\pi$ gives an isomorphism of Z-modules from $L \cap \bar{L}$ to $L / q_{1}^{-1} L$.

Definition 8.16. An integral crystal basis of $\mathbf{U}^{+}$is a $\mathbf{Z}\left[q_{1}^{-1}\right]$-basis of $L$ that coincides with the canonical basis $\mathbf{B}$ of $\mathbf{U}^{+}$modulo $q_{1}^{-1} L$.

Theorem 8.17. Let $B$ be the $\mathbf{Z}\left[q_{1}, q_{1}^{-1}\right]$-basis of $\mathbf{U}_{\mathbf{Z}}^{+}$in Theorem 8.14 (2). Then, $B$ is an integral crystal basis of $\mathbf{U}^{+}$.

Proof. By the quasi-orthonormality, $B$ is a $\mathbf{Z}\left[q_{1}^{-1}\right]$-basis of $L$; hence, $\pi(B)$ is a $\mathbf{Z}$-basis of $L / q_{1}^{-1} L$; thus, the transformation matrix with coefficients in $\mathbf{Z}$ between $\pi(B)$ and $\pi(\mathbf{B})$ can be taken as the identity up to signs. We have to show that all the signs are plus. For that purpose, by virtue of $[\mathrm{L} 4,8.3]$, it is enough to show that any $\mathbf{S}_{\lambda}$ belongs to $\mathbf{B}+q_{1}^{-1} L$. We argue by the induction on the length of $\lambda$, which is denoted by $l(\lambda)$. First, assume that $l(\lambda)=0,1$. Then, $\mathbf{S}_{\lambda}=P_{s}$ for some $s \geq 0$. By a close look at Theorem 8.5 with $r=2 s$ and by Proposition 6.4 together with Lemma 6.12 , we have $E_{\alpha_{0}}^{(s)} E_{\alpha_{1}}^{(2 s)} \equiv P_{s}$ $\bmod q_{1}^{-1} L$. By Proposition $8.15((4),(5))$, we have $E_{\alpha_{0}}^{(s)} E_{\alpha_{1}}^{(2 s)} \in \mathbf{B}+q_{1}^{-1} L$; hence, $P_{s} \in \mathbf{B}+q_{1}^{-1} L$. Now, assume that $l(\lambda) \geq 2$. By the same argument in the proof of [BCP, Lemma 4.2], the induction proceeds.

\section{Appendix A. Additional Commutation Relations}

We shall study the commutation relations between the real root vectors of height 2 and -2 (Corollary A.3).

Lemma A.1. Let $k, l \geq 0$. Then,

$$
\begin{aligned}
& {\left[E_{\alpha_{0}}, E_{k \delta+\alpha_{1}} E_{l \delta+\alpha_{1}}\right]_{q^{-4}}} \\
& =\left(q_{1}-q_{1}^{-1}\right) \sum_{i=0}^{l} q^{-2} b_{2(l-i)+1} E_{k \delta+\alpha_{1}} \tilde{\psi}_{i} E_{(l-i+1) \delta-\alpha_{1}} \\
& \quad+\left(q_{1}-q_{1}^{-1}\right) \sum_{i=0}^{k} q^{-1} b_{2(k-i)+1} E_{l \delta+\alpha_{1}} \tilde{\psi}_{i} E_{(k-i+1) \delta-\alpha_{1}}
\end{aligned}
$$




$$
\begin{aligned}
& +\left(q_{1}-q_{1}^{-1}\right)\left(1+q^{-1}\right) \sum_{i=0}^{k-1} \sum_{j=1}^{k-i} q^{-1} b_{2 i-1} b_{2(k-i-j+1)} E_{(k+l-i-j+1) \delta+\alpha_{1}} \tilde{\psi}_{i} E_{j \delta-\alpha_{1}} \\
& +\left(q_{1}-q_{1}^{-1}\right) \sum_{i=1}^{k+1} b_{2 i-1} \tilde{\psi}_{k-i+1} \tilde{\psi}_{l+1} .
\end{aligned}
$$

Proof. Using Proposition 3.26 ((2), (4), (7)), we have

$$
\begin{aligned}
E_{\alpha_{0}} E_{k \delta+\alpha_{1}} E_{l \delta+\alpha_{1}} & \left(E_{k \delta+\alpha_{1}} E_{\alpha_{0}} q^{-2}+\left(q_{1}-q_{1}^{-1}\right) \sum_{i=0}^{k} b_{2(k-i)+1} \tilde{\psi}_{i} E_{(k-i+1) \delta-\alpha_{1}}\right) E_{l \delta+\alpha_{1}} \\
= & E_{k \delta+\alpha_{1}}\left(E_{l \delta+\alpha_{1}} E_{\alpha_{0}} q^{-2}+\left(q_{1}-q_{1}^{-1}\right) \sum_{i=0}^{l} b_{2(l-i)+1} \tilde{\psi}_{i} E_{(l-i+1) \delta-\alpha_{1}}\right) q^{-2} \\
& +\left(q_{1}-q_{1}^{-1}\right) \sum_{i=0}^{k} b_{2(k-i)+1} \tilde{\psi}_{i}\left(E_{l \delta+\alpha_{1}} E_{(k-i+1) \delta-\alpha_{1}} q^{-1}+\tilde{\psi}_{k+l-i+1}\right) \\
= & E_{k \delta+\alpha_{1}} E_{l \delta+\alpha_{1}} E_{\alpha_{0}} q^{-4}+\left(q_{1}-q_{1}^{-1}\right) \sum_{i=0}^{l} q^{-2} b_{2(l-i)+1} E_{k \delta+\alpha_{1}} \tilde{\psi}_{i} E_{(l-i+1) \delta-\alpha_{1}} \\
& +\left(q_{1}-q_{1}^{-1}\right) \sum_{i=0}^{k} q^{-1} b_{2(k-i)+1}\left(E_{l \delta+\alpha_{1}} \tilde{\psi}_{i}+\left(1+q^{-1}\right) \sum_{j=0}^{i-1} b_{2(i-j)}\right. \\
& \left.\times E_{(l+i-j) \delta+\alpha_{1}} \tilde{\psi}_{j} \theta(i \geq 1)\right) E_{(k-i+1) \delta-\alpha_{1}} \\
& +\left(q_{1}-q_{1}^{-1}\right) \sum_{i=0}^{k} b_{2(k-i)+1} \tilde{\psi}_{i} \tilde{\psi}_{k+l-i+1} .
\end{aligned}
$$

Rewriting the double summation, we obtain the lemma.

Proposition A.2. Let $n \geq 1$. Then,

$$
\begin{aligned}
{\left[E_{\alpha_{0}}, E_{2 n \delta-\alpha_{0}}\right]_{q^{-4}}[4]_{1}=} & \sum_{i=0}^{n-1} \sum_{j=1}^{n-i} E_{(n+j-1) \delta+\alpha_{1}} \tilde{\psi}_{i} E_{(n-i-j+1) \delta-\alpha_{1}} \\
& \times b_{2 j-1} b_{2(n-j)+1}\left(q-q^{-3}\right)\left(q_{1}-q_{1}^{-1}\right) \\
& +\left(\tilde{\psi}_{n}^{2}+\sum_{i=1}^{n} \tilde{\psi}_{n-i} \tilde{\psi}_{n+i}\left(b_{2 i+1}-q b_{2 i-1}\right)\right)\left(q_{1}-q_{1}^{-1}\right)
\end{aligned}
$$


Proof. By Corollary 3.10, we have

$$
\begin{aligned}
{\left[E_{\alpha_{0}}, E_{2 n \delta-\alpha_{0}}\right]_{q^{-4}}[4]_{1}=} & {\left[E_{\alpha_{0}}, E_{n \delta+\alpha_{1}} E_{(n-1) \delta+\alpha_{1}}\right]_{q^{-4}} } \\
& -q\left[E_{\alpha_{0}}, E_{(n-1) \delta+\alpha_{1}} E_{n \delta+\alpha_{1}}\right]_{q^{-4}} .
\end{aligned}
$$

Applying Lemma A.1, we have

$$
\begin{aligned}
{\left[E_{\alpha_{0}},\right.} & \left.E_{2 n \delta-\alpha_{0}}\right]_{q^{-4}}[4]_{1}\left(q_{1}-q_{1}^{-1}\right)^{-1} \\
= & \sum_{i=0}^{n-1} q^{-2} b_{2(n-i)-1} E_{n \delta+\alpha_{1}} \tilde{\psi}_{i} E_{(n-i) \delta-\alpha_{1}} \\
& +\sum_{i=0}^{n} q^{-1} b_{2(n-i)+1} E_{(n-1) \delta+\alpha_{1}} \tilde{\psi}_{i} E_{(n-i+1) \delta-\alpha_{1}} \\
& +\left(1+q^{-1}\right) \sum_{i=0}^{n-1} \sum_{j=1}^{n-i} q^{-1} b_{2 j-1} b_{2(n-i-j+1)} E_{(2 n-i-j) \delta+\alpha_{1}} \tilde{\psi}_{i} E_{j \delta-\alpha_{1}} \\
& +\sum_{i=1}^{n+1} b_{2 i-1} \tilde{\psi}_{n-i+1} \tilde{\psi}_{n+i-1} \\
& -q\left(\sum_{i=0}^{n} q^{-2} b_{2(n-i)+1} E_{(n-1) \delta+\alpha_{1}} \tilde{\psi}_{i} E_{(n-i+1) \delta-\alpha_{1}}\right. \\
& +\sum_{i=0}^{n-1} q^{-1} b_{2(n-i)-1} E_{n \delta+\alpha_{1}} \tilde{\psi}_{i} E_{(n-i) \delta-\alpha_{1}} \\
& +\left(1+q^{-1}\right) \sum_{i=0}^{n-2} \sum_{j=1}^{n-i-1} q^{-1} b_{2 j-1} b_{2(n-i-j)} E_{(2 n-i-j) \delta+\alpha_{1}} \tilde{\psi}_{i} E_{j \delta-\alpha_{1}} \\
& \left.+\sum_{i=1}^{n} b_{2 i-1} \tilde{\psi}_{n-i} \tilde{\psi}_{n+i}\right) .
\end{aligned}
$$

The second term and the fifth one cancel out. Combining the first one and the sixth one, and then adding it to the seventh one for $j=n-i, 1 \leq i \leq n-1$, we have

$$
\begin{aligned}
& {\left[E_{\alpha_{0}}, E_{2 n \delta-\alpha_{0}}\right]_{q^{-4}}[4]_{1}\left(q_{1}-q_{1}^{-1}\right)^{-1}} \\
& =\left(1+q^{-1}\right) \sum_{i=0}^{n-1} \sum_{j=1}^{n-i} b_{2 j-1}\left(q^{-1} b_{2(n-i-j+1)}-b_{2(n-i-j)}\right) E_{(2 n-i-j) \delta+\alpha_{1}} \tilde{\psi}_{i} E_{j \delta-\alpha_{1}} \\
& \quad+\sum_{i=1}^{n+1} b_{2 i-1} \tilde{\psi}_{n-i+1} \tilde{\psi}_{n+i-1}-q \sum_{i=1}^{n} b_{2 i-1} \tilde{\psi}_{n-i} \tilde{\psi}_{n+i} .
\end{aligned}
$$

Applying Lemma 3.14 (2) to the first term, we obtain the proposition. 
Corollary A.3. Let $m \geq 0, n \geq 1$. Then,

$$
\begin{aligned}
{\left[E_{2 m \delta+\alpha_{0}}, E_{2 n \delta-\alpha_{0}}\right]_{q^{-4}}[4]_{1} } \\
=\sum_{i=0}^{m+n-1} \sum_{j=1}^{m+n-i} E_{(n+j-1) \delta+\alpha_{1}} \tilde{\psi}_{i} E_{(2 m+n-i-j+1) \delta-\alpha_{1}} \\
\quad \times b_{2 j-1} b_{2(m+n-j)+1}\left(q-q^{-3}\right)\left(q_{1}-q_{1}^{-1}\right) \\
\quad+\left(\tilde{\psi}_{m+n}^{2}+\sum_{i=1}^{m+n} \tilde{\psi}_{m+n-i} \tilde{\psi}_{m+n+i}\left(b_{2 i+1}-q b_{2 i-1}\right)\right)\left(q_{1}-q_{1}^{-1}\right) .
\end{aligned}
$$

Proof. Applying $T_{\varpi_{1}}^{m}$ to Proposition A.2 with $n$ replaced by $n+m$, we obtain the corollary.

\section{Appendix B. Connection with the Drinfeld Generators}

Definition B.1 [Dr]. Let $\mathbf{D}$ be the $\mathbf{Q}\left(q_{1}\right)$-algebra generated by

$$
\left\{x_{n}^{ \pm}, a_{k}, K^{ \pm 1}, C^{ \pm 1 / 2} \mid n \in \mathbf{Z}, k \in \mathbf{Z} \backslash\{0\}\right\}
$$

with the following defining relations:

(D1) $C^{ \pm 1 / 2}$ are central, $C^{1 / 2} C^{-1 / 2}=1, K K^{-1}=K^{-1} K=1$,

(D2) $\left[a_{k}, K\right]=0, K x_{n}^{ \pm} K^{-1}=q^{ \pm 1} x_{n}^{ \pm}$,

(D3) $\left[a_{k}, x_{n}^{ \pm}\right]= \pm \frac{[2 k]_{1}}{k}\left(q^{k}+q^{-k}+(-1)^{k+1}\right) C^{\mp|k| / 2} x_{n+k}^{ \pm}$,

(D4) $\left[a_{k}, a_{l}\right]=\delta_{k+l, 0} \frac{[2 k]_{1}}{k}\left(q^{k}+q^{-k}+(-1)^{k+1}\right) \frac{C^{k}-C^{-k}}{q_{1}-q_{1}^{-1}}$,

(D5) $\left[x_{n}^{+}, x_{m}^{-}\right]=\frac{C^{(n-m) / 2} \psi_{n+m}^{+}-C^{(m-n) / 2} \psi_{n+m}^{-}}{q_{1}-q_{1}^{-1}}$,

(D6) $\operatorname{Sym}_{n, m}\left(x_{n+2}^{ \pm} x_{m}^{ \pm}+\left(q^{\mp 1}-q^{ \pm 2}\right) x_{n+1}^{ \pm} x_{m+1}^{ \pm}-q^{ \pm 1} x_{n}^{ \pm} x_{m+2}^{ \pm}\right)=0$,

(D7) $\operatorname{Sym}_{k, l, m}\left(q_{1}^{3} x_{k \mp 1}^{ \pm} x_{l}^{ \pm} x_{m}^{ \pm}-\left(q_{1}+q_{1}^{-1}\right) x_{k}^{ \pm} x_{l \mp 1}^{ \pm} x_{m}^{ \pm}+q_{1}^{-3} x_{k}^{ \pm} x_{l}^{ \pm} x_{m \mp 1}^{ \pm}\right)=0$,

(D8) $\operatorname{Sym}_{k, l, m}\left(q_{1}^{-3} x_{k \pm 1}^{ \pm} x_{l}^{ \pm} x_{m}^{ \pm}-\left(q_{1}+q_{1}^{-1}\right) x_{k}^{ \pm} x_{l \pm 1}^{ \pm} x_{m}^{ \pm}+q_{1}^{3} x_{k}^{ \pm} x_{l}^{ \pm} x_{m \pm 1}^{ \pm}\right)=0$.

Here, each suffix runs through all the possible cases and we set $\psi_{n}^{+}=0$ for $n \leq$ $-1, \psi_{n}^{-}=0$ for $n \geq 1$, and

$$
\sum_{i \geq 0} \psi_{ \pm i}^{ \pm} u^{i}=K^{ \pm 1} \exp \left( \pm\left(q_{1}-q_{1}^{-1}\right) \sum_{j \geq 1} a_{ \pm j} u^{j}\right) .
$$


Definition B.2. Let $\mathbf{U}_{\mathbf{D}}$ be the $\mathbf{Q}\left(q_{1}\right)$-subalgebra of $\mathbf{U}$ generated by $e_{i}, f_{i}, k_{i}^{ \pm 1}, c^{ \pm 1 / 2}:=q^{ \pm\left(h_{0}+2^{-1} h_{1}\right)}$ for $i \in I$.

Proposition B.3. The following gives an isomorphism of $\mathbf{Q}\left(q_{1}\right)$ algebras from $\mathbf{D}$ to $\mathbf{U}_{\mathbf{D}}$ :

$$
\begin{aligned}
& C^{1 / 2} \mapsto c^{1 / 2}, K \mapsto k_{1}, \\
& x_{n}^{+} \mapsto T_{\varpi_{1}}^{-n}\left(e_{1}\right) \text { for } n \in \mathbf{Z}, \\
& x_{n}^{-} \mapsto T_{\varpi_{1}}^{n}\left(f_{1}\right) \text { for } n \in \mathbf{Z}, \\
& a_{k} \mapsto c^{-k / 2} E_{k \delta} \text { for } k \geq 1, \\
& a_{-k} \mapsto c^{k / 2} \Omega\left(E_{k \delta}\right) \text { for } k \geq 1, \\
& \psi_{n}^{+} \mapsto\left(q_{1}-q_{1}^{-1}\right) c^{-n / 2} k_{1} \tilde{\psi}_{n} \text { for } n \geq 0, \\
& \psi_{-n}^{-} \mapsto-\left(q_{1}-q_{1}^{-1}\right) c^{n / 2} k_{1}^{-1} \Omega\left(\tilde{\psi}_{n}\right) \text { for } n \geq 0 .
\end{aligned}
$$

Here, we set

$$
\left(q_{1}-q_{1}^{-1}\right) \sum_{n \geq 1} E_{n \delta} u^{n}=\log \left(1+\left(q_{1}-q_{1}^{-1}\right) \sum_{k \geq 1} \tilde{\psi}_{k} u^{k}\right),
$$

or equivalently,

$$
n E_{n \delta}=n \tilde{\psi}_{n}-\left(q_{1}-q_{1}^{-1}\right) \sum_{k=1}^{n-1} k E_{k \delta} \tilde{\psi}_{n-k} \theta(n \geq 2) \quad \text { for } n \geq 1 .
$$

The inverse map is given by

$$
\begin{aligned}
& c^{1 / 2} \mapsto C^{1 / 2}, k_{1} \mapsto K, k_{0} \mapsto C K^{-2}, e_{1} \mapsto x_{0}^{+}, f_{1} \mapsto x_{0}^{-}, \\
& e_{0} \mapsto[4]_{1}^{-1} q^{-1} C K^{-2}\left[x_{0}^{-}, x_{1}^{-}\right]_{q}, f_{0} \mapsto[4]_{1}^{-1} q C^{-1}\left[x_{-1}^{+}, x_{0}^{+}\right]_{q^{-1}} K^{2} .
\end{aligned}
$$

Sketch of Proof: Using the results in Section 3 and the Q-linear antiinvolution $\Omega$ of $\mathbf{U}$, we can directly check that the correspondence $(\star)$ is consistent with (D1)-(D6). To check (D3), we use the following identity:

$$
\begin{aligned}
\left(1+q^{-1}\right) n b_{2 n}= & \left(1+q^{-1}\right) \sum_{i=1}^{n} b_{2(n-i)}\left(q^{i}-q^{-i}\right)\left(q^{i}+q^{-i}+(-1)^{i+1}\right) \\
& +\left(q^{n}-q^{-n}\right)\left(q^{n}+q^{-n}+(-1)^{n+1}\right) q^{-2} \quad \text { for } n \geq 1 .
\end{aligned}
$$

We can also check (D7) and (D8), which will be written elsewhere. Thus, we obtain the surjective $\mathbf{Q}\left(q_{1}\right)$-algebra homomorphism from $\mathbf{D}$ to $\mathbf{U}_{\mathbf{D}}$. Its injectivity follows from the specialization argument. See $[\mathrm{B} 1]$ and $[J]$ for the related matter. 


\section{References}

[B1] Beck, J., Braid group action and quantum affine algebras, Comm. Math. Phys., 165 (1994), 555-568.

[B2] $\longrightarrow$, Convex bases of PBW type for quantum affine algebras, Comm. Math. Phys., 165 (1994), 193-199.

[BCP] Beck, J., Chari, V. and Pressley, A., An algebraic characterization of the affine canonical basis, Duke Math. J., 99 (1999), 455-487.

[CP] Chari, V. and Pressley, A., Quantum affine algebras at roots of unity, Represent. Theory, 1 (1997), 280-328.

[Da1] Damiani, I., A basis of type Poincaré-Birkhoff-Witt for the quantum algebra of $\widehat{s l}(2)$, J. Algebra, 161 (1993), 291-310.

[Da2] - The R-matrix for (twisted) affine quantum algebras, Representations and Quantizations, China High Educ. Press, Beijing, 2000, 89-144.

[Dr] Drinfeld, V., A new realization of Yangians and quantized affine algebras, Soviet Math. Dokl., 36 (1988), 212-216.

[GL] Grojnowski, I. and Lusztig, G., A comparison of bases of quantized enveloping algebras, Linear algebraic groups and their representations, Contemp. Math., 153 (1993), 11-19.

[J] Jing, N., On Drinfeld realization of quantum affine algebras, The Monster and Lie Algebras, Ohio State Univ. Math. Res. Inst. Publ., 7 (1998), 195-206.

[K1] Kashiwara, M., On crystal bases of the $q$-analogue of universal enveloping algebras, Duke Math. J., 63 (1991), 465-516.

[K2] - On level zero representations of quantized affine algebras, to appear.

[KhT] Khoroshkin, S. M. and Tolstoy, V. N., The uniqueness theorem for the universal R-matrix, Lett. Math. Phys., 24 (1992), 231-244.

[L1] Lusztig, G., Finite dimensional Hopf algebras arising from quantized universal enveloping algebras, J. Amer. Math. Soc., 3 (1990), 257-296.

[L2] Canonical bases arising from quantized enveloping algebras, J. Amer. Math. Soc., 3 (1990), 447-498.

[L] - Introduction to Quantum Groups, Birkhäuser, Boston, 1993.

[L4] Braid group action and canonical bases, Adv. Math., 122 (1996), 237-261.

[M] Macdonald, I. G., Symmetric Functions and Hall Polynomials, 2nd ed., Oxford University Press, New York, 1995. 